Universidad Autónoma Metropolitana-Iztapalapa

División de Ciencias Básicas e Ingeniería

Departamento de Matemáticas

\title{
Completitud en el Sentido de Čech y sus Generalizaciones
}

\author{
Miguel López DE LunA \\ Tesis Doctoral
}

Asesor: Dr. Vladimir Tkachuk

enero del 2002, México D.F. 


\section{Contenido}

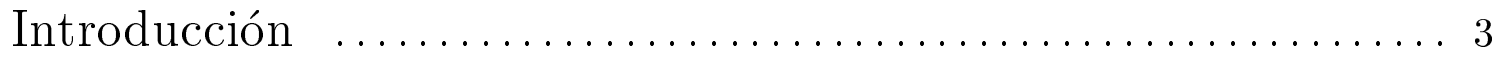

\section{Capítulo 1. Preliminares}

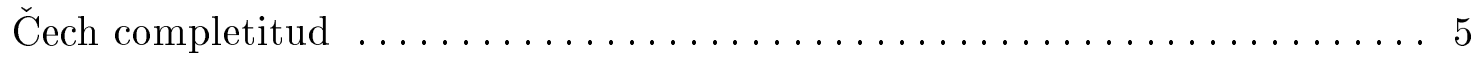

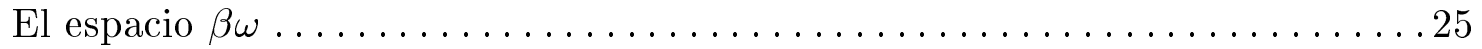

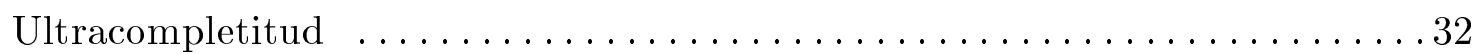

Capítulo 2. Aditividad y algunas otras propiedades de la Čech completitud

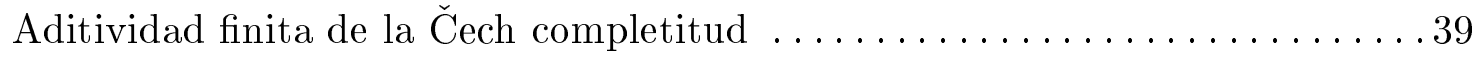

La no aditividad de la Čech completitud

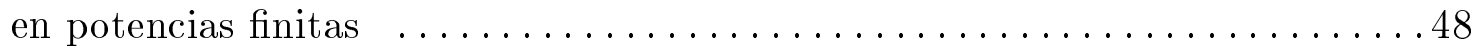

\section{Capítulo 3. Ultracompletitud}

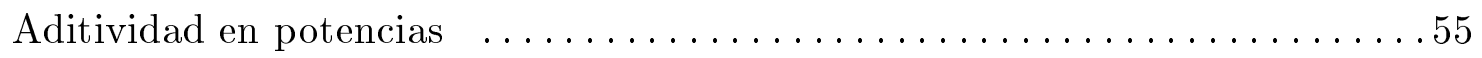

Un ejemplo de un espacio numerablemente compacto,

Čech completo y no ultracompleto

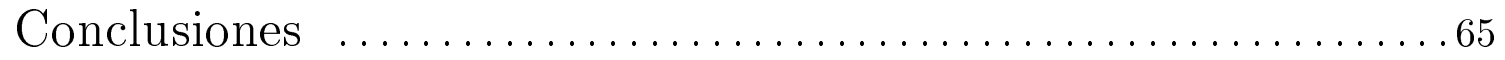

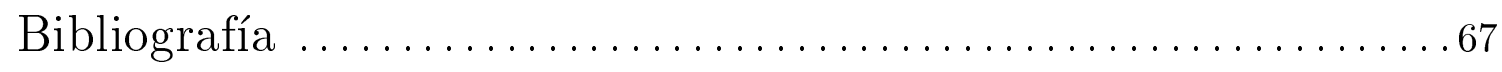

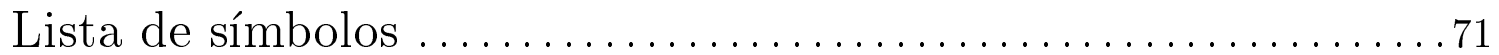




\section{Introducción}

En el Análisis, existe la noción de espacio completo. Decimos que un espacio métrico $(X, d)$ es completo (o Cauchy completo) si toda sucesión de Cauchy converge en $X$. Este concepto es de gran utilidad para demostrar teoremas importantes, como por ejemplo, el famoso Teorema del Punto Fijo. La completitud según Čech es una generalización de la completitud métrica a la clase de los espacios de Tychonoff. Un espacio topológico $X$ de Tychonoff se llama Čech completo si $X$ es un subespacio $G_{\delta}$ en toda compactificación $c X$ de $X$. Naturalmente, cuando un espacio $X$ es completamente metrizable es equivalente a decir que $X$ es metrizable y Čech completo.

Los espacios Čech completos fueron definidos por Čech en 1937. Posteriormente, la caracterización interna de la Čech completitud fue dada, de manera independiente, por Frolík [Fr3] en el año 1960 y por Arhangel'skiǔ [Ar1] en 1961.

Otra propiedad que también hemos estudiado, es la noción de espacio ultracompleto. Decimos que un espacio topológico $X$ de Tychonoff es ultracompleto si $X$ tiene carácter numerable en toda compactificación $c X$ de $X$. Claramente, la ultracompletitud es una propiedad más fuerte que la Čech completitud y ambas son consecuencia de la compacidad local. En 1987 Ponomarev y Tkachuk introdujeron en [PT] el concepto de espacios fuertemente completos, como aquellos espacios $X$ que poseen una base numerable en $\beta X$. En [BY1], esta propiedad fue llamada ultracompletitud y se proporcionó una caracterización interna de los espacios ultracompletos.

En este trabajo se presentan algunos resultados obtenidos sobre los espacios Čech completos y los espacios ultracompletos. En particular, en lo que se refiere a la aditividad de estas propiedades, se aportan resultados nuevos en espacios topológicos y en grupos topológicos.

En el capítulo 1 se presenta una introducción muy breve de la Čech completitud y de la ultracompletitud, en donde aparecen las definiciones y resultados básicos que serán usados en los capítulos posteriores. Se proporciona un ejemplo de un espacio no Čech completo que posee una cubierta puntualmente finita de subespacios abiertos y Čech completos [CČN]. También se introduce el concepto de espacio casi Čech completo y se demuestra su invarianza respecto a funciones abiertas; lo cual será esencial para demostrar la preservación de la Čech completitud en grupos topológicos. En este capítulo también se estudia el espacio $\beta \omega$ y algunas de sus propiedades elementales.

El capítulo 2 está dedicado fundamentalmente a estudiar la aditividad de la 
Čech completitud. Se dice que una propiedad $\mathcal{P}$ de un espacio $X$ es ( resp. finitamente aditiva en potencias numerables) aditiva en la potencia $n \in \mathbb{N}$ si $X \in \mathcal{P}$ siempre que ( resp. $\left.X^{\omega}=\bigcup_{i=1}^{n} X_{i}\right) X^{n}=\bigcup_{i=1}^{n} X_{i}$ donde $X_{i} \in \mathcal{P}$. En [Tk] se demuestra la aditividad en potencias finitas para muchas propiedades que no son aditivas. Entre las contribuciones de nuestro trabajo, proporcionamos un ejemplo en que la Čech completitud no es aditiva en potencias finitas en algunos modelos de ZFC. Probamos que la Čech completitud es finitamente aditiva en potencias numerables, al igual que la propiedad de tipo puntual numerable. Por último, probamos algunas propiedades de la Čech completitud en grupos topológicos, en particular, mostramos que la Čech completitud es aditiva en este caso.

En el capítulo 3 proporcionamos varios resultados relativos a la ultracompletitud. Usamos una caracterización de la ultracompletitud dada en [PT] para probar, entre otras cosas, que la compacidad local y la ultracompletitud son propiedades equivalentes en grupos topológicos. Establecemos que la ultracompletitud en espacios métricos es aditiva en potencias finitas y que si $X^{\omega}=\bigcup_{i \in \omega} X_{i}$ con $X_{i}$ ultracompleto entonces $X$ es ultracompleto. En el año 2000 Buhagiar y Yoshioka preguntaron en [BY2] si todo espacio Čech completo y numerablemente compacto es ultracompleto. Bajo $\mathrm{CH}$ nosotros respondemos a esta pregunta, exhibiendo un espacio no ultracompleto, Čech completo y numerablemente compacto.

Todos los resultados contenidos en este trabajo fueron expuestos y comentados en el Seminario de Topología dirigido por el Dr. Vladimir Tkachuk en el Departamento de Matemáticas de la Universidad Autónoma Metropolitana-Iztapalapa y en el Seminario de Topología dirigido por el Dr. A. Tamariz Mascarúa en el Departamento de Matemáticas de la Facultad de Ciencias de la UNAM. La mayor parte de estos resultados fue presentada también en el Primer Encuentro México-Japón de Topología y sus Aplicaciones en Morelia Mex. y en el Congreso de Verano de Topología General en Nueva York EEUU, ambos eventos en el año 1999.

Por último, deseo manifestar mi agradecimiento al Dr. Vladimir Tkachuk por su dirección y enseñanzas en la elaboración de esta tesis. 


\section{Capítulo 1}

\section{Preliminares}

El objetivo principal de este capítulo es presentar una introducción muy breve de la Čech completitud, la ultracompletitud y del espacio $\beta \omega$. Estos resultados serán la referencia básica a los capítulos 2 y 3 , que son la parte medular de este trabajo.

Todos los espacios considerados se supondrán de Tychonoff a menos que se especifique otra cosa.

\section{Čech completitud.}

Sea $X$ un espacio topológico. Decimos que el par $\left(X^{\prime}, c\right)$, donde $X^{\prime}$ es un espacio compacto y $c: X \rightarrow X^{\prime}$ es una inmersión tal que $\overline{c(X)}=X^{\prime}$, es una compactificación del espacio $X$. Observemos que si existe una inmersión $f: X \rightarrow f(X) \subset X^{\prime}$, donde $X^{\prime}$ es un espacio compacto, entonces $(\overline{f(X)}, i \circ f)$ es una compactificación de $X$, donde $i$ denota la inmersión de $f(X)$ en $\overline{f(X)}$. De aquí resulta que todo espacio que puede ser inmerso en un espacio compacto, tiene una compactificación. Por lo tanto, un espacio topológico $X$ es de Tychonoff si y sólo si el espacio $X$ tiene una compactificación. A la compactificación $\left(X^{\prime}, c\right)$ del espacio $X$ la denotaremos por $c X$.

Observemos que si tenemos dos compactificaciones $c_{1} X, c_{2} X$ de $X$ y funciones continuas $f_{1}: c_{1} X \rightarrow c_{2} X$ y $f_{2}: c_{2} X \rightarrow c_{1} X$ que satisfacen $f_{1} \circ c_{1}=c_{2}$ y $f_{2} \circ c_{2}=c_{1}$, entonces $c_{1} X$ y $c_{2} X$ son espacios homeomorfos. Como $f_{2} \circ f_{1} \circ c_{1}=f_{2} \circ c_{2}=c_{1}$, se cumple que $f_{2} \circ f_{1}\left\lceil c_{1}(X)=I_{1}\left\lceil c_{1}(X)\right.\right.$, donde $I_{1}: c_{1} X \rightarrow c_{1} X$ es la función identidad. Esto nos conduce a que $f_{2} \circ f_{1}=I_{1}$, pues ambas funciones coinciden en el conjunto denso $c_{1}(X) \subset c_{1} X$. De manera similar, obtenemos la igualdad $f_{1} \circ f_{2}=I_{2}$. De modo que $f_{1}$ es un homeomorfismo. Haciendo $f=f_{1}$ se cumple lo pedido.

Diremos que las compactificaciones $c_{1} X$ y $c_{2} X$ de $X$ son equivalentes si existe un homeomorfismo $f: c_{1} X \rightarrow c_{2} X$ tal que $f \circ c_{1}=c_{2}$. De la observación anterior se desprende que $c_{1} X$ y $c_{2} X$ son equivalentes si existen funciones continuas $f_{1}$ : $c_{1} X \rightarrow c_{2} X$ y $f_{2}: c_{2} X \rightarrow c_{1} X$ tales que $f_{1} \circ c_{1}=c_{2}$ y $f_{2} \circ c_{2}=c_{1}$.

La mayoría de los resultados que aparecen en esta sección, pueden ser consultados en alguna de las siguientes referencias: $[\mathrm{Ap}],[\mathrm{En}],[\mathrm{Ho} 3],[\mathrm{Ke}] \mathrm{y}[\mathrm{Na}]$. 
1.1. Lema (Lema de Shura-Bura). Sea $X$ un espacio compacto y $\gamma \subset \exp (X)$. Sea $F=\bigcap\{\bar{A}: A \in \gamma\}$. Luego, para todo $U \in \mathcal{T}(F, X)$ existen $A_{1}, A_{2}, \ldots, A_{n} \in \gamma$ tales que $\overline{A_{1}} \cap \overline{A_{2}} \cap \ldots \cap \overline{A_{n}} \subset U$.

Demostración. Consideremos la familia $\mu=\{\bar{A} \cap(X-U): A \in \gamma\}$ de subconjuntos cerrados de $X$. Dado que $F \subset U$ obtenemos

$$
\bigcap \mu=\bigcap\{\bar{A} \cap(X-U): A \in \gamma\}=F \cap(X-U)=\emptyset .
$$

Puesto que $X$ es compacto, la familia $\mu$ no es centrada. De aquí se sigue que existe una colección finita $A_{1}, A_{2}, \ldots, A_{n} \in \gamma$ tal que

$$
\left(\overline{A_{1}} \cap(X-U)\right) \cap\left(\overline{A_{2}} \cap(X-U)\right) \cap \ldots \cap\left(\overline{A_{n}} \cap(X-U)\right)=\overline{A_{1}} \cap \ldots \cap \overline{A_{n}} \cap(X-U)=\emptyset
$$

lo cual implica $\overline{A_{1}} \cap \ldots \cap \overline{A_{n}} \subset U$.

1.2. Proposición. Sean $A$ un subespacio denso de un espacio topológico $X$ y $f: A \rightarrow Z$ una función continua, donde $Z$ es un espacio compacto. La función $f$ tiene una extensión continua sobre $X$ si y sólo si para todo par de subconjuntos cerrados y ajenos $B_{1}, B_{2}$ de $Z$, las imagenes inversas $f^{-1}\left(B_{1}\right)$ y $f^{-1}\left(B_{2}\right)$ tienen cerraduras ajenas en el espacio $X$.

Demostración. Sea $F: X \rightarrow Z$ una extensión continua de $f$. Si $B_{i}=\overline{B_{i}}$ para $i=1,2$ y $B_{1} \cap B_{2}=\emptyset$, entonces $F^{-1}\left(B_{i}\right)=\overline{F^{-1}\left(B_{i}\right)}$ cuando $i \in\{1,2\}$ y $F^{-1}\left(B_{1}\right) \cap$ $F^{-1}\left(B_{2}\right)=\emptyset$. De aquí se desprende que

$$
\overline{f^{-1}\left(B_{1}\right)} \cap \overline{f^{-1}\left(B_{2}\right)} \subset F^{-1}\left(B_{1}\right) \cap F^{-1}\left(B_{2}\right)=\emptyset .
$$

Ahora probemos que la condición en el teorema también es suficiente. Para cada $x \in$ $X$ consideremos la familia $\mathcal{F}(x)=\{\overline{f(A \cap U)}\}_{U \in \mathcal{T}(x, X)}$ de subconjuntos cerrados de $Z$. Observemos que $\mathcal{F}(x)$ es centrada. En efecto, para $U_{1}, U_{2}, \ldots, U_{k} \in \mathcal{T}(x, X)$ se tiene que

$$
\emptyset \neq \overline{f\left(A \cap U_{1} \cap U_{2} \cdots \cap U_{k}\right)} \subset \overline{f\left(A \cap U_{1}\right)} \cap \overline{f\left(A \cap U_{2}\right)} \cap \overline{f\left(A \cap U_{k}\right)} .
$$

Como el espacio $Z$ es compacto, tenemos que $\bigcap \mathcal{F}(x) \neq \emptyset$ para todo $x \in X$. Probemos que $|\bigcap \mathcal{F}(x)|=1$; lo cual implicará en particular que $\bigcap \mathcal{F}(x)=\{f(x)\}$ para $x \in A$. Supongamos que $y_{1}, y_{2} \in \bigcap \mathcal{F}(x)$ e $y_{1} \neq y_{2}$. Existen $V_{1} \in \mathcal{T}\left(y_{1}, X\right)$ y $V_{2} \in \mathcal{T}\left(y_{2}, X\right)$ tales que $\overline{V_{1}} \cap \overline{V_{2}}=\emptyset$. Por hipótesis tenemos que $\overline{f^{-1}\left(V_{1}\right)} \cap$ $\overline{f^{-1}\left(V_{2}\right)}=\emptyset$. Sin perder la generalidad, podemos suponer que $x \notin \overline{f^{-1}\left(V_{1}\right)}$. Luego, $X-\overline{f^{-1}\left(V_{1}\right)} \in \mathcal{T}(x, X)$ e $y_{1} \in \bigcap \mathcal{F}(x) \subset \overline{f\left(A-\overline{f^{-1}\left(V_{1}\right)}\right)}$. Por otro lado, como $V_{1} \cap f\left(A-\overline{f^{-1}\left(V_{1}\right)}\right)=\emptyset$, obtenemos que $y_{1} \notin \overline{f\left(A-\overline{f^{-1}\left(V_{1}\right)}\right)}$, lo cual es una contradicción. 
Consideremos la función $F: X \rightarrow Z$ dada por $\{F(x)\}=\bigcap \mathcal{F}(x)$. De la observación hecha anteriormente se sigue que $F\lceil A=f$. Para terminar nuestra demostración, verifiquemos que la función $F$ es continua.

Sea $V \in \mathcal{T}(F(x), Z)$. Como $\{F(x)\}=\bigcap_{U \in \mathcal{T}(x, X)} \overline{f(A \cap U)} \subset V$, por el lema de Shura-Bura, existe una familia $\left\{U_{1}, U_{2}, \ldots, U_{k}\right\} \subset \mathcal{T}(x, X)$ tal que

$$
\overline{f\left(A \cap U_{1}\right)} \cap \overline{f\left(A \cap U_{2}\right)} \cap \overline{f\left(A \cap U_{k}\right)} \subset V .
$$

Dado que $U_{1} \cap U_{2} \cap \cdots \cap U_{k}=U \in \mathcal{T}(x, X)$ y por (1) y (2) concluimos que $F\left(x^{\prime}\right) \in \overline{f(A \cap U)} \subset V$, para todo $x^{\prime} \in U$, es decir, $F(U) \subset V$.

1.3. Teorema. Sean $c_{1} X$ y $c_{2} X$ compactificaciones de un espacio $X$. Entonces $c_{1} X$ y $c_{2} X$ son equivalentes si y sólo si para todo par de subconjuntos cerrados $A, B \subset X$ se cumple

$$
\overline{c_{1}(A)} \cap \overline{c_{1}(B)}=\emptyset \Longleftrightarrow \overline{c_{2}(A)} \cap \overline{c_{2}(B)}=\emptyset .
$$

Demostración. Si las compactificaciones $c_{1} X$ y $c_{2} X$ son equivalentes, entonces claramente se satisface $(*)$. Supóngase que para las compactificaciones $c_{1} X$ y $c_{2} X$ es válido $(*)$, donde $A, B \subset X$ son cerrados.

De acuerdo a la proposición 1.2, las funciones $c_{1} \circ h_{2}: c_{2}(X) \rightarrow c_{1} X$ y $c_{2} \circ h_{1}$ : $c_{1}(X) \rightarrow c_{2} X$, donde $h_{i}$ es la inversa de $c_{i}: X \rightarrow c_{i}(X)$ para $i=1,2$, se pueden extender a funciones $f_{2}: c_{2} X \rightarrow c_{1} X$ y $f_{1}: c_{1} X \rightarrow c_{2} X$. Como $f_{2} \circ c_{2}=c_{1} \mathrm{y}$ $f_{1} \circ c_{1}=c_{2}$, tenemos que $c_{1} X$ y $c_{2} X$ son equivalentes.

Sea $X$ un espacio de Tychonoff e $I=[0,1]$. Consideremos el conjunto $A=$ $\{\alpha: X \rightarrow I: \alpha$ es continua $\}$. Para cada $x \in X$ definimos $h(x) \in I^{A}$ de la manera siguiente: $h(x)(\alpha)=\alpha(x)$. De este modo tenemos una función $h: X \rightarrow I^{A}$. Verifiquemos que la función $h$ es un homeomorfismo entre $X$ y $h(X)$. Sean $x, y \in X$ con $x \neq y$. Existe una función continua $\alpha: X \rightarrow I$ tal que $\alpha(x)=1$ y $\alpha(y)=0$. Como $\alpha \in A$, tenemos que $\alpha(x)=h(x)(\alpha) \neq \alpha(y)=h(y)(\alpha)$, así que $h(x) \neq h(y)$. Por lo tanto $h$ es una inyección sobre $h(X)$. La continuidad de la función $h$ se sigue de la igualdad $p_{\alpha} \circ h=\alpha$, donde la función $p_{\alpha}$ es la proyección sobre el $\alpha$ ésimo factor del producto $I^{A}$. Sea $U \in \mathcal{T}^{*}(X)$. Probemos que $h(U)$ es un conjunto abierto en $h(X)$. Tomemos $x \in U$. Existe una función continua $\alpha: X \rightarrow I$ tal que $\alpha(x)=0$ y $\alpha(X-U)=\{1\}$. El conjunto abierto $V=h(X) \cap p_{\alpha}^{-1}([0,1))$ satisface $h(x) \in V \subset h(U)$. En efecto, sea $y \in V$. Luego, $y=h(z) \in p_{\alpha}^{-1}([0,1))$ para algún $z \in X$. De modo que $\left(p_{\alpha} \circ h\right)(z)=\alpha(z) \neq 1$. Por lo tanto $z \in U$, es decir, $y \in h(U)$. De la igualdad $\left(p_{\alpha} \circ h\right)(x)=\alpha(x)=0$ resulta que $h(x) \in V$. Por lo tanto $h$ es una función abierta y con ello se prueba que $h$ es un homeomorfismo.

Consideremos el conjunto compacto $\beta X=\overline{h(X)} \subset I^{A}$. Identificando $X$ y $h(X)$, podemos afirmar que $X$ está contenido en $\beta X$ y $\bar{X}=\beta X$. La compactificación $\beta X$ del espacio $X$ recibe el nombre de compactificación de Stone-Čech de $X$. 
1.4. Teorema. Toda función continua $f: X \rightarrow Y$ de un espacio de Tychonoff $X$ a un espacio compacto $Y$, se extiende a una única función continua $\widehat{f}: \beta X \rightarrow Y$. Si toda función continua de un espacio de Tychonoff $X$ a un espacio compacto es extendible a una función continua en una compactificación $\alpha X$ de $X$, entonces $\alpha X$ es equivalente a la compactificación de Stone-Čech de $X$.

Demostración. Consideremos el conjunto $A$ dado en la construcción de $\beta X$ y $A^{\prime}=\{\alpha: \alpha: Y \rightarrow I$ es una función continua $\}$. Sean $h_{X}: X \rightarrow I^{A}$ y $h_{Y}: Y \rightarrow I^{A^{\prime}}$ las inmersiones tales que $\beta X=\overline{h_{X}(X)}$ y $\beta Y=\overline{h_{Y}(Y)}$. Para cada $h \in I^{A}$ definimos $F(h) \in I^{A^{\prime}}$ como $F(h)(\alpha)=h(\alpha \circ f)$. De manera que tenemos un función $F: I^{A} \rightarrow$ $I^{A^{\prime}}$. De la igualdad $p_{\alpha} \circ F=p_{\alpha \circ f}$ se desprende que $F$ es una función continua. Sea $x \in X$. Luego $\left(F \circ h_{X}\right)(x)(\alpha)=F\left(h_{X}(x)\right)(\alpha)=h_{X}(\alpha \circ f)=(\alpha \circ f)(x)$. Por otro lado tenemos que $\left(h_{Y} \circ f\right)(x)(\alpha)=h_{Y}(f(x))(\alpha)=(\alpha \circ f)(x)$. De aquí se sigue que $F \circ h_{X}=h_{Y} \circ f$. Por lo tanto $F\left(h_{X}(X)\right) \subset h_{Y}(f(X))=h_{Y}(Y)$. Como $F$ es continua, obtenemos que $F(\beta X)=F\left(\overline{\left.h_{X}(X)\right)} \subset \overline{F\left(h_{X}(X)\right)} \subset \overline{h_{Y}(Y)}=\beta Y\right.$. Así que la restricción $F_{\beta}=F\left\lceil\beta X\right.$ también verifica $F_{\beta} \circ h_{X}=h_{Y} \circ f$. Como $Y$ es compacto, tenemos que $h_{Y}: Y \rightarrow \beta Y$ es un homeomorfismo. Luego, la función continua $\widehat{f}=h_{Y}{ }^{-1} \circ F_{\beta}$ satisface $\widehat{f} \circ h_{X}=h_{Y}{ }^{-1} \circ F_{\beta} \circ h_{X}=f$. Dado que las funciones continuas $f$ y $\widehat{f}$ coinciden en un denso de $\beta X$, se desprende que la extensión $\widehat{f}$ es única.

Supongamos que la compactificación $\alpha X$ de $X$ tiene la propiedad dada en la segunda parte del teorema. Luego, existe una extensión continua $f: \alpha X \rightarrow \beta X$ de la inmersión $\beta: X \rightarrow \beta X$. Por lo tanto $f \circ \alpha=\beta$. Como también es válida la igualdad $\widehat{f} \circ \beta=\alpha$, se sigue que las compactificaciones $\alpha X$ y $\beta X$ son equivalentes.

Dos subconjuntos $A$ y $B$ de un espacio $X$, son llamados completamente separados si existe una función continua $f: X \rightarrow I=[0,1]$ tal que $f(A) \subset\{0\}$ y $f(B) \subset\{1\}$; decimos que $f$ separa a los conjuntos $A$ y $B$.

Del teorema anterior obtenemos los siguientes corolarios importantes.

1.5. Corolario. Dos conjuntos completamente separados de un espacio de Tychonoff $X$, tienen cerraduras ajenas en $\beta X$. Si una compactificación $\alpha X$ de un espacio de Tychonoff $X$ tiene la propiedad que todo par de subconjuntos completamente separados del espacio $X$ tienen cerraduras ajenas en $\alpha X$, entonces $\alpha X$ es equivalente a $\beta X$.

Demostración. Sean $A$ y $B$ conjuntos completamente separados de un espacio de Tychonoff $X$ y sea $f: X \rightarrow I$ una función continua que separa a $A$ y $B$. Por el teorema anterior, existe una extensión continua $F: \beta X \rightarrow I$ de la función $f$. Dado que $A \subset F^{-1}(0)$ y $B \subset F^{-1}(1)$, obtenemos que $\bar{A}^{\beta X} \cap \bar{B}^{\beta X}=\emptyset$.

Supongamos que $\alpha X$ es una compactificación de $X$ con la propiedad dada en la segunda parte del corolario. Notemos que en virtud del lema de Urysohn, si $A, B \subset$ 
$X$ satisfacen $\bar{A}^{\alpha X} \cap \bar{B}^{\alpha X}=\emptyset$, entonces los conjuntos $A$ y $B$ son completamente separados. Por lo tanto, los únicos subconjuntos de $X$ con cerraduras ajenas en $\alpha X$ son los conjuntos completamente separados. Lo mismo es válido para $\beta X$. Por lo tanto, del teorema 1.3 se desprende que $\alpha X$ y $\beta X$ son equivalentes.

1.6. Corolario. Toda función continua $f: X \rightarrow I$ de un espacio de Tychonoff $X$ al intervalo $I=[0,1]$, se extiende a una función continua $F: \beta X \rightarrow I$. Si toda función continua de un espacio de Tychonoff $X$ al intervalo $I$ es extendible a una función continua en una compactificación $\alpha X$ de $X$, entonces $\alpha X$ es equivalente a la compactificación de Stone-Čech de X.

1.7. Corolario. Dos subconjuntos cerrados y ajenos de un espacio normal $X$, tienen cerraduras ajenas en $\beta X$. Si una compactificación $\alpha X$ de un espacio de Tychonoff $X$ tiene la propiedad que todo par de subconjuntos cerrados y ajenos del espacio $X$ tienen cerraduras ajenas en $\alpha X$, entonces $X$ es normal y $\alpha X$ es equivalente a $\beta X$.

Sean $X$ y $Y$ espacios de Tychonoff, $c X$ y $c^{\prime} Y$ compactificaciones de $X$ y $Y$ respectivamente, y sea $f: X \rightarrow Y$ una función continua. Si existe una función continua $F: c X \rightarrow c^{\prime} Y$ para la cual $F(x)=f(x)$ para todo $x \in X$, entonces decimos que $f$ es continuamente extendible sobre las compactificaciones $c X$ y $c^{\prime} Y$ y llamamos a $F$ una extensión de $f$ sobre $c X$ y $c^{\prime} Y$. Este concepto nos permite formular la siguiente propiedad importante de la compactificación de Stone-Čech.

1.8. Corolario. Para toda compactificación $\alpha Y$ de un espacio de Tychonoff $Y$ y toda función continua $f: X \rightarrow Y$ donde $X$ es un espacio de Tychonoff, existe una extensión continua $F$ de $f$ sobre $\beta X$ y $\alpha Y$.

1.9. Corolario. Si un subespacio $M \subset X$, donde $X$ es un espacio de Tychonoff, tiene la propiedad que toda función continua $f: M \rightarrow I$ es continuamente extendible sobre $X$, entonces la cerradura $\bar{M}^{\beta X}$ es una compactificación de $M$ equivalente a $\beta M$. Además, si $M$ es denso en $X$, entonces $\beta M=\beta X$.

El último corolario implica

1.10. Corolario. Para todo espacio de Tychonoff $X$ y cualquier espacio $T$ tal que $X \subset T \subset \beta X$ se cumple $\beta T=\beta X$.

Sea $c X$ una compactificación del espacio $X$. El conjunto $c X-c(X)$ es llamado el residuo de la compactificación $c X$. La propiedad más importante del residuo está dada por el teorema 1.12; para su demostración necesitaremos del siguiente lema.

1.11. Lema. Tomemos un subespacio denso $A$ de un espacio de Hausdorff $X$ y consideremos la función continua $f: X \rightarrow Y$, donde $Y$ es cualquier espacio 
topológico. Si la restricción $f\lceil A: A \rightarrow f(A) \subset Y$ es un homeomorfismo entonces $f(X-A) \cap f(A)=\emptyset$.

Demostración. Supongamos que $f(x) \in f(A)$ para algún $x \in X-A$. Podemos suponer sin pérdida de generalidad que $X=A \cup\{x\}$ y que $Y=f(A)$. Sea $y \in A$ para el cual $f(x)=f(y)$. Elijamos $U \in \mathcal{T}(x, X), V \in \mathcal{T}(y, X)$ tales que $U \cap V=\emptyset$. El conjunto $f(A-V)=f \uparrow_{A}(A-V)$ es cerrado en $Y=f(A)$, y por lo tanto $f^{-1} f(A-V)=A-V$ es cerrado en $X$. Luego $X-(A-V)=\{x\} \cup V$ es un conjunto abierto. Por lo tanto $(\{x\} \cup V) \cap U=\{x\} \in \mathcal{T}(X)$, así que $x \notin \bar{A}=X$, lo que es una contradicción.

1.12. Teorema. Si $c_{1} X$ y $c_{2} X$ son compactificaciones de un espacio $X$ de $T y$ chonoff y la función continua $f: c_{1} X \rightarrow c_{2} X$ satisface $f \circ c_{1}=c_{2}$, entonces

$$
f\left(c_{1}(X)\right)=c_{2}(X) \text { y } f\left(c_{1} X-c_{1}(X)\right)=c_{2} X-c_{2}(X) .
$$

Demostración. La primera igualdad se sigue de la condición $f \circ c_{1}=c_{2}$; la segunda es consecuencia de la primera, del lema anterior y del hecho que $f\left(c_{1} X\right)=c_{2} X$.

El siguiente teorema será la base de la definición de la Čech completitud.

1.13 Teorema. Sea $X$ un espacio de Tychonoff. Las siguientes condiciones son equivalentes:

(i) Para toda compactificación $c X$ de $X$, el residuo $c X-c(X)$ es un conjunto $F_{\sigma}$ en $c X$.

(ii) El residuo $\beta X-\beta(X)$ es un conjunto $F_{\sigma}$ en $\beta X$.

(iii) Existe una compactificación $c X$ de $X$ tal que el residuo $c X-c(X)$ es un conjunto $F_{\sigma}$ en $c X$.

Demostración. Las implicaciones (i) $\Longrightarrow$ (ii) y (ii) $\Longrightarrow$ (iii) son obvias. Probemos (iii) $\Longrightarrow($ ii). El teorema 1.4 nos asegura la existencia de una función continua $f$ : $\beta X \rightarrow c X$ tal que $f \circ \beta=c$. Por el teorema anterior, tenemos que $f^{-1}(c X-c(X))=$ $\beta X-\beta(X)$. Dado que por hipótesis el residuo $c X-c(X)$ es un subconjunto $F_{\sigma}$ de $c X$, obtenemos que $\beta X-\beta(X)$ es un conjunto $F_{\sigma}$ en $\beta X$.

Ahora demostremos (ii) $\Longrightarrow\left(\right.$ i). Sea $\beta X-\beta(X)=\bigcup_{n \in \mathbb{N}} F_{n}$, donde cada conjunto $F_{i}$ es cerrado en $\beta X$. Consideremos una compactificación $c X$ del espacio $X$ y una función $f: \beta X \rightarrow c X$ para la cual $f \beta=c$. Del teorema anterior deducimos que $c X-c(X)=\bigcup_{n \in \mathbb{N}} f\left(F_{n}\right)$. Puesto que los conjuntos $f\left(F_{n}\right)$ son cerrados en $c X$, tenemos que $c X-c(X)$ es un conjunto $F_{\sigma}$ en $c X$.

Un espacio topológico $X$ de Tychonoff se llama Čech completo si $X$ satisface cualquiera de las condiciones del teorema anterior. 
Es evidente que todo espacio compacto es Čech completo. Si $X$ es localmente compacto entonces $X$ es también Čech completo, ya que todo espacio no compacto y localmente compacto posee una compactificación cuyo residuo consiste de un sólo punto. Los números irracionales son un ejemplo de un espacio Čech completo y no localmente compacto.

La definición de la Čech completitud de $X$ es una definición externa; está dada en términos de su relación con otros espacios, sus compactificaciones $c X$. El teorema 1.15 nos proporciona varias caracterizaciones internas de la Čech completitud, obtenidas por A. V. Arhangel'skiu [Ar1].

Dado un espacio $X$ y $\mathcal{F} \subset \exp (X)$, denotaremos por $\cap \mathcal{F}=\cap\{F: F \in \mathcal{F}\}$ y $\cap \overline{\mathcal{F}}=\cap\{\bar{F}: F \in \mathcal{F}\}$.

1.14. Definición. Una familia $\mathcal{F} \subset \exp (X)$ se llama dominada por una sucesión de cubiertas abiertas $\mathcal{U}=\left\{\mathcal{U}_{n}\right\}_{n \in \mathbb{N}}$, si para todo $n \in \mathbb{N}$ existen $F \in \mathcal{F}, U \in \mathcal{U}_{n}$ tales que $F \subset U$.

1.15. Teorema. Las siguientes condiciones son equivalentes:

(i) $X$ es Čech completo;

(ii) Existe una sucesión $\left\{\mathcal{U}_{n}\right\}_{n \in \mathbb{N}}$ de cubiertas abiertas de $X$ tal que si $\mathcal{F} \subset \exp (X)$ es una familia centrada con $\mathcal{U}_{n} \cap \mathcal{F} \neq \emptyset$ para todo $n \in \mathbb{N}$, entonces $\cap \overline{\mathcal{F}} \neq \emptyset$;

(iii) Existe una sucesión $\left\{\mathcal{U}_{n}\right\}_{n \in \mathbb{N}}$ de cubiertas abiertas de $X$ tal que si $\mathcal{F} \subset \exp (X)$ es una base de filtro con $\mathcal{U}_{n} \cap \mathcal{F} \neq \emptyset$ para todo $n \in \mathbb{N}$, entonces $\bigcap \overline{\mathcal{F}} \neq \emptyset$;

(iv) Existe una sucesión $\left\{\mathcal{U}_{n}\right\}_{n \in \mathbb{N}}$ de cubiertas abiertas de $X$ tal que si $\mathcal{F} \subset \exp (X)$ es un filtro con $\mathcal{U}_{n} \cap \mathcal{F} \neq \emptyset$ para todo $n \in \mathbb{N}$, entonces $\bigcap \overline{\mathcal{F}} \neq \emptyset$;

(v) Existe una sucesión $\left\{\mathcal{U}_{n}\right\}_{n \in \mathbb{N}}$ de cubiertas abiertas de $X$ tal que si $\mathcal{F} \subset \exp (X)$ es un ultrafiltro con $\mathcal{U}_{n} \cap \mathcal{F} \neq \emptyset$ para todo $n \in \mathbb{N}$, entonces $\bigcap \overline{\mathcal{F}} \neq \emptyset$;

(vi) Existe una sucesión $\mathcal{U}=\left\{\mathcal{U}_{n}\right\}_{n \in \mathbb{N}}$ de cubiertas abiertas de $X$ tal que si $\mathcal{F} \subset$ $\exp (X)$ es una familia centrada dominada por $\mathcal{U}$, entonces $\bigcap \overline{\mathcal{F}} \neq \emptyset$;

(vii) Existe una sucesión $\mathcal{U}=\left\{\mathcal{U}_{n}\right\}_{n \in \mathbb{N}}$ de cubiertas abiertas de $X$ tal que si $\mathcal{F} \subset \exp (X)$ es una base de filtro dominada por $\mathcal{U}$, entonces $\bigcap \overline{\mathcal{F}} \neq \emptyset$;

(viii) Existe una sucesión $\mathcal{U}=\left\{\mathcal{U}_{n}\right\}_{n \in \mathbb{N}}$ de cubiertas abiertas de $X$ tal que si $\mathcal{F} \subset \exp (X)$ es un filtro dominado por $\mathcal{U}$, entonces $\bigcap \overline{\mathcal{F}} \neq \emptyset$;

(ix) Existe una sucesión $\mathcal{U}=\left\{\mathcal{U}_{n}\right\}_{n \in \mathbb{N}}$ de cubiertas abiertas de $X$ tal que si $\mathcal{F} \subset$ $\exp (X)$ es una familia centrada de subconjuntos cerrados dominada por $\mathcal{U}$, entonces $\bigcap \mathcal{F} \neq \emptyset$

(x) Existe una sucesión $\mathcal{U}=\left\{\mathcal{U}_{n}\right\}_{n \in \mathbb{N}}$ de cubiertas abiertas de $X$ tal que si $\mathcal{F} \subset$ $\exp (X)$ es una base de filtro de subconjuntos cerrados dominada por $\mathcal{U}$, entonces $\bigcap \mathcal{F} \neq \emptyset$.

Demostración. (i) $\Longrightarrow$ (ii). Tenemos por hipótesis que $X=\bigcap_{n \in \mathbb{N}} U_{n}$ donde $U_{n} \in$ $\mathcal{T}(\beta X)$ para todo $n \in \mathbb{N}$. Para cada $n \in \mathbb{N}$, consideremos la familia $\mathcal{U}_{n}=\{V \in$ 
$\left.\mathcal{T}(X): \bar{V}^{\beta X} \subset U_{n}\right\}$. Probemos que $\mathcal{U}_{n}$ es una cubierta abierta de X para todo $n \in \mathbb{N}$. Sea $n \in \mathbb{N}$. Es inmediato que $\mathcal{U}_{n} \subset \mathcal{T}(X)$. Sea $x \in X$. Luego, $x \in U_{n}$. Por regularidad, existe $W \in \mathcal{T}(x, \beta X)$ tal que $W \subset \bar{W}^{\beta X} \subset U_{n}$. Por lo tanto $x \in X \cap W=V \in \mathcal{T}(X)$. Por ser $X$ un subespacio denso de $\beta X$ resulta que $\bar{V}^{\beta X}={\overline{X \cap W^{\beta X}}}^{\beta X} \bar{W}^{\beta X} \subset U_{n}$. De aquí se desprende que $V \in \mathcal{U}_{n}$. Por lo tanto $x \in V \in \mathcal{U}_{n}$, es decir, $X=\bigcup \mathcal{U}_{n}$.

Afirmamos que $\left\{\mathcal{U}_{n}\right\}_{n \in \mathbb{N}}$ satisface la propiedad (ii). Sea $\mathcal{F}=\left\{F_{s}\right\}_{s \in S} \subset \exp (X)$ una familia centrada tal que $\mathcal{F} \cap \mathcal{U}_{n} \neq \emptyset$ para todo $n \in \mathbb{N}$. Probemos que $\bigcap \overline{\mathcal{F}} \neq \emptyset$. Como $\overline{\mathcal{F}}^{\beta X}$ es una familia centrada de cerrados en $\beta X$ y $\beta X$ es compacto, obtenemos que $\bigcap^{\beta X} \neq \emptyset$. Sea $x \in \bigcap \overline{\mathcal{F}}^{\beta X}$. Para terminar nuestra demostración, es sufiente mostrar que $x \in X$, ya que $\bigcap \overline{\mathcal{F}}=\bigcap\{\bar{F}: F \in \mathcal{F}\}=\bigcap\left\{\bar{F}^{\beta X} \cap X: F \in \mathcal{F}\right\}=$ $\cap \overline{\mathcal{F}}^{\beta X} \cap X$. Tomando en cuenta que $x \in{\overline{F_{s_{n}}}}^{\beta X}$ donde $F_{s_{n}} \in \mathcal{F} \cap \mathcal{U}_{n}$ con $n \in \mathbb{N}$, deducimos que $x \in{\overline{F_{s_{n}}}}^{\beta X} \subset U_{n}$ para todo $n \in \mathbb{N}$. Por lo tanto $x \in \bigcap_{n \in \mathbb{N}} U_{n}=X$.

La verificación de (ii) $\Longrightarrow$ (iii) $\Longrightarrow$ (iv) $\Longrightarrow$ (v) se sigue de la relación:

$(*) \mathcal{F}$ es ultrafiltro $\Longrightarrow \mathcal{F}$ es filtro $\Longrightarrow \mathcal{F}$ es base de filtro $\Longrightarrow \mathcal{F}$ es familia centrada

Para demostrar $(\mathrm{v}) \Longrightarrow$ (vi), verifiquemos que la sucesión $\left\{\mathcal{U}_{n}\right\}_{n \in \mathbb{N}}$ de cubiertas abiertas de $X$ dada por hipótesis, satisface (vi). Consideremos una familia centrada $\mathcal{F} \subset \exp (X)$, tal que para todo $n \in \mathbb{N}$ existen $F \in \mathcal{F}, U \in \mathcal{U}_{n}$ con $F \subset U$. Sea $\gamma$ un ultrafiltro en $X$ para el cual $\mathcal{F} \subset \gamma$. Dado que $\gamma$ es ultrafiltro, si $H \in \mathcal{F} \subset \gamma$ y $H \subset U$ entonces $U \in \gamma$. De aquí se sigue que $\gamma \cap \mathcal{U}_{n} \neq \emptyset$ para todo $n \in \mathbb{N}$. Luego, por (v) obtenemos $\bigcap \bar{\gamma} \neq \emptyset$. Del hecho de que $\bigcap \bar{\gamma} \subset \bigcap^{\mathcal{F}}$ se desprende que $\bigcap \overline{\mathcal{F}} \neq \emptyset$.

La demostración de (vi) $\Longrightarrow$ (vii) $\Longrightarrow$ (viii) se obtiene al emplear $(*)$.

Probemos (viii) $\Longrightarrow$ (ix). La sucesión $\left\{\mathcal{U}_{n}\right\}$ de cubiertas abiertas de $X$ proporcionada por (viii) cumple la necesidad. En efecto, sea $\mathcal{F} \subset \exp (X)$ una familia centrada de cerrados, tal que para todo $n \in \mathbb{N}$ existen $H \in \mathcal{F}$ y $U \in \mathcal{U}_{n}$ para los cuales $H \subset U$. Consideremos un filtro $\gamma$ para el cual $\mathcal{F} \subset \gamma$. Tenemos que $\gamma \cap \mathcal{U}_{n} \neq \emptyset$ para todo $n \in \mathbb{N}$. De la hipótesis se desprende que $\bigcap \bar{\gamma} \neq \emptyset$. Por lo tanto $\bigcap \mathcal{F} \neq \emptyset$, ya que $\bigcap \bar{\gamma} \subset \bigcap \mathcal{F}$.

Para probar (ix) $\Longrightarrow(\mathrm{x})$ es suficiente notar que toda base de filtro es familia centrada.

Probemos $(\mathrm{x}) \Longrightarrow(\mathrm{i})$. Antes de proceder con la demostración, establezcamos lo siguiente. Sea $n \in \mathbb{N}$. Consideremos la cubierta abierta $\mathcal{U}_{n}$ de $X$, proporcionada por la hipótesis. Para cada $U \in \mathcal{U}_{n}$ existe $\widehat{U} \in \mathcal{T}(\beta X)$ para el cual $U=X \cap \widehat{U}$. Tomemos $U_{n}=\bigcup_{U \in \mathcal{U}_{n}} \widehat{U} \in \mathcal{T}(\beta X)$. Demostremos que $X=\bigcap_{n \in \mathbb{N}} U_{n}$. Fijemos 
$x \in X$ y tomemos un $n \in \mathbb{N}$. Luego, $x \in U$ para algún $U \in \mathcal{U}_{n}$. Como $U=X \cap \widehat{U}$ se sigue que $x \in \widehat{U}$. Por lo tanto $x \in U_{n}$. Lo anterior implica que $X \subset \bigcap_{n \in \mathbb{N}} U_{n}$. Ahora probemos que $\bigcap_{n \in \mathbb{N}} U_{n} \subset X$. Supongamos que $\left(\bigcap_{n \in \mathbb{N}} U_{n}\right)-X \neq \emptyset$ y tomemos un $z \in\left(\bigcap_{n \in \mathbb{N}} U_{n}\right)-X$. Consideremos la familia $\mathcal{F}=\left\{\overline{U \cap X}^{X}: U \in\right.$ $\mathcal{T}(z, \beta X)\}$. Es evidente que $\mathcal{F}$ es base de filtro en $X$.

Observemos que $\mathcal{F}$ está dominada por $\mathcal{U}$. En efecto, sea $n \in \mathbb{N}$. Luego, $z \in U_{n}$, de donde $z \in \widehat{U} \in \mathcal{T}(\beta X)$ para algún $U \in \mathcal{U}_{n} \operatorname{con} U=\widehat{U} \cap X$. Existe $V \in \mathcal{T}(z, \beta X)$ tal que $V \subset \bar{V}^{\beta X} \subset \widehat{U}$. De aquí $\overline{X \cap V}{ }^{X}=\overline{X \cap V}^{\beta X} \cap X=\bar{V}^{\beta X} \cap X \subset U$. El conjunto $F=\overline{X \cap V}^{X}$ pertenece a $\mathcal{F}$ y además $F \subset U \in \mathcal{U}_{n}$. Si $\cap \mathcal{F} \neq \emptyset$, sea $y \in \bigcap \mathcal{F}$. Existen $V \in \mathcal{T}(z, \beta X)$ y $W \in \mathcal{T}(y, \beta X)$ tales que $V \cap W=\emptyset$. Por lo tanto $y \notin F$, pues $y \in X \cap W \in \mathcal{T}(X)$ y $(X \cap W) \cap V=\emptyset$. Así que $\cap \mathcal{F}=\emptyset$, lo que constituye una contradicción para $(\mathrm{x})$. De modo que nuestra suposición resultó ser falsa y por lo tanto $\bigcap_{n \in \mathbb{N}} U_{n} \subset X$.

1.16. Definición. La sucesión $\mathcal{U}=\left\{\mathcal{U}_{n}\right\}_{n \in \mathbb{N}}$ de cubiertas abiertas $X$ se llama completa si satisface cualquiera de las condiciones de (ii) a (x) del teorema anterior.

Según la definición anterior podemos afirmar que el espacio $X$ es Čech completo si y sólo si $X$ posee una sucesión completa de cubiertas abiertas.

1.17. Proposición. Sean $X$ un espacio Čech completo y $\mathcal{B}$ una base de $X$. Si $\mathcal{U}=\left\{\mathcal{U}_{n}\right\}_{n \in \mathbb{N}}$ es una sucesión completa de cubiertas abiertas de $X$, entonces $\mathcal{B}^{\prime}=$ $\left\{\mathcal{B}_{n}\right\}_{n \in \mathbb{N}}$ es una sucesión completa de cubiertas abiertas de $X$, donde $\mathcal{B}_{n}=\{B \in$ $\mathcal{B}: B \subset U$ para algún $\left.U \subset \mathcal{U}_{n}\right\}$ para todo $n \in \mathbb{N}$.

Demostración. Sea $n \in \mathbb{N}$. Es obvio que $\mathcal{B}_{n} \subset \mathcal{T}(X)$. Probemos que $\mathcal{B}_{n}$ es cubierta de $X$. Tomemos un $x \in X$. Luego, $x \in U$ para algún $U \in \mathcal{U}_{n}$. Existe $B \in \mathcal{B}$ tal que $x \in U \in \mathcal{B}_{n}$. Así que $x \in \mathcal{B}_{n}$ y por lo tanto $X=\bigcup \mathcal{B}_{n}$. Probemos que $\mathcal{B}^{\prime}$ satisface la condición (ix) del teorema 1.15. Sea $\mathcal{F} \subset \exp (X)$ una familia centrada de cerrados dominada por $\mathcal{B}^{\prime}$. Luego, para todo $n \in \mathbb{N}$ existen $F \in \mathcal{F}, B \in \mathcal{B}_{n}$ y $U \in \mathcal{U}_{n}$ para los cuales $F \subset B \subset U$. De la completitud de $\mathcal{U}$ se sigue que $\bigcap \mathcal{F} \neq \emptyset$.

Este resultado $[\mathrm{AL}]$ nos permite suponer, sin pérdida de generalidad, que todos los elementos de cualquier sucesión completa de cubiertas abiertas de $X$ son subfamilias de una base dada.

1.18. Teorema. Sea $X$ un espacio $T_{1}$ y primero numerable con un sólo punto $x_{0}$ no aislado. Entonces $X$ es Čech completo.

Demostración. Observemos que $X$ es un espacio normal. Sean $A$ y $B$ subconjuntos cerrados y ajenos de $X$. Para el caso en que $x_{0} \notin A \cup B$, tomemos los abiertos $U=A$ y $V=B$ los cuales satisfacen $A \subset U, B \subset V$ y $U \cap V=\emptyset$. En caso contrario 
podemos suponer, sin pérdida de generalidad, que $x_{0} \in A$ y por lo tanto $B \in \mathcal{T}(X)$. Por consiguiente, los subconjuntos abiertos y ajenos $U=X-B$ y $V=B$ son tales que $A \subset U$ y $B \subset V$. De lo anterior se concluye que $X$ es normal y por ser $T_{1}$, el espacio $X$ es de Tychonoff. Para concluir nuestra demostración, establezcamos que $X$ es un subconjunto $G_{\delta}$ de $\beta X$. Dado que $X$ es primero numerable en $x_{0}$, también lo es el espacio $\beta X$. En efecto, sea $\left\{U_{n}\right\}_{n \in \mathbb{N}} \subset \mathcal{T}(X)$ una base en $x_{0}$. Para cada $n \in \mathbb{N}$, tomemos $V_{n} \in \mathcal{T}(\beta X)$ tal que $U_{n}=X \cap V_{n}$. Probemos que la familia $\mathcal{B}=\left\{V_{n}\right\}_{n \in \mathbb{N}} \subset \mathcal{T}(\beta X)$ es una base en $x_{0}$. Sea $V \in \mathcal{T}\left(x_{0}, \beta X\right)$. Existe $U \in \mathcal{T}(\beta X)$ para el cual $x_{0} \in U \subset \bar{U} \subset V$. Luego $x_{0} \in X \cap V_{n_{0}} \subset X \cap U$. De aquí se desprende que $x_{0} \in V_{n_{0}} \subset \overline{V_{n_{0}}}=\overline{X \cap U}=\bar{U} \subset V$ y por lo tanto $\mathcal{B}$ es base en $x_{0}$. Puesto que $\left\{x_{0}\right\}=\bigcap_{n \in \mathbb{N}} V_{n}$ obtenemos $X=\bigcap_{n \in \mathbb{N}}\left(X \cup V_{n}\right)$. Debido a que $X \cup V_{n} \in \mathcal{T}(\beta X)$ para todo $n \in \mathbb{N}$, el espacio $X$ es un subconjunto $G_{\delta}$ de $\beta X$.

1.19. Teorema. La Čech completitud se hereda a subconjuntos cerrados y a subconjuntos $G_{\delta}$.

Demostración. Sea $X$ un espacio Čech completo. Consideremos el subconjunto cerrado $F \subset X$. Existe una sucesión completa $\mathcal{U}=\left\{\mathcal{U}_{n}\right\}_{n \in \mathbb{N}}$ de cubiertas abiertas de $X$. Sea $\mathcal{U}_{F}=\left\{\mathcal{U}_{n}^{F}\right\}_{n \in \mathbb{N}}$ donde $\mathcal{U}_{n}^{F}=\left\{F \cap U: U \in \mathcal{U}_{n}\right\}$. Afirmamos que $\mathcal{U}_{F}$ satisface (ix) de 1.15. Es obvio que $\mathcal{U}_{n}^{F} \subset \mathcal{T}(F)$ y $F=\bigcup \mathcal{U}_{n}^{F}$ para todo $n \in \mathbb{N}$. Consideremos la familia centrada $\mathcal{F} \subset \exp (F)$ cuyos elementos son subconjuntos cerrados de $F$, y por lo tanto de $X$. Supóngase que $\mathcal{F}$ está dominada por $\mathcal{U}_{F}$. Luego, para todo $n \in \mathbb{N}$ existen $U^{F} \in \mathcal{U}_{n}^{F}$ y $K \in \mathcal{F}$ con $K \subset U^{F}$. Dado que $U^{F}=F \cap U$ para algún $U \in \mathcal{U}_{n}$, obtenemos que $K \subset U$. Por lo tanto $\mathcal{U}$ domina a $\mathcal{F}$ y como $\mathcal{U}$ es completa, la familia $\mathcal{F}$ verifica $\bigcap \mathcal{F} \neq \emptyset$.

Para la segunda parte del teorema, sea $G$ un subconjunto $G_{\delta}$ de $X$. Dado que $X$ es un subconjunto $G_{\delta}$ de $c X$, donde $c X$ es cualquier compactificación de $X$, el conjunto $G$ es un subconjunto $G_{\delta}$ de $c X$, y por lo tanto de $\bar{G}^{c X}$, que es una compactificación de $G$.

1.20. Teorema. La suma $\bigoplus_{s \in S} X_{s}$ es Čech completo si y sólo si cada espacio $X_{s}$ es Čech completo.

Demostración. Sea $X=\bigoplus_{s \in S} X_{s}$ un espacio Čech completo. Puesto que cada $X_{s}$ es abierto y cerrado en $X$, el espacio $X_{s}$ es Čech completo para todo $s \in S$. Supongamos ahora que todos lo espacios $X_{s}$ son Čech completos. Para cada $s \in S$ tomemos una compactificación $c_{s} X_{s}$ del espacio $X_{s}$. Luego $c_{s} X_{s}-X_{s}=\bigcup_{i \in \mathbb{N}} F_{s, i}$, donde $F_{s, i}$ son subconjuntos cerrados de $c_{s} X_{s}$ para todo $s \in S$ e $i \in \mathbb{N}$. Dado que la suma discreta de espacios localmente compactos es un espacio localmente compacto, se sigue que $X=\bigoplus_{s \in S} c_{s} X_{s}$ es localmente compacto y por lo tanto Čech completo. Como $X-\bigoplus_{s \in S} X_{s}=\bigcup_{i \in \mathbb{N}}\left(\bigcup_{s \in S} F_{s, i}\right)$ y los conjuntos $\bigcup_{s \in S} F_{s, i}$ son cerrados en $X$ para todo $i \in \mathrm{N}$, tenemos que la suma $\bigoplus_{s \in S} X_{s}$ es un espacio Čech completo. 
1.21. Proposición. Si $f: X \rightarrow Y$ es una función perfecta, entonces para cualquier conjunto $B \subset Y$, la función $f_{B}: f^{-1}(B) \rightarrow B$ que asigna a cada $x \in f^{-1}(B)$ el punto $f(x) \in B$, es perfecta.

Demostración. Tomemos un conjunto cerrado $A \subset f^{-1}(B)$. De las igualdades

$$
f_{B}\left(A \cap f^{-1}(B)\right)=f\left(A \cap f^{-1}(B)\right)=f(A) \cap B
$$

se desprende que $f_{B}$ es cerrada. Dado que $f_{B}^{-1}(y)=f^{-1}(y)$ con $y \in B$, la fibra $f_{B}^{-1}(y)$ es un conjunto compacto para todo $y \in B$.

1.22. Lema (Teorema de Kliushin). Una función perfecta y sobre $f: X \rightarrow Y$ no se puede extender continuamente sobre un espacio $Z$ de Hausdorff para el cual $Z-X \neq \emptyset$ y $\bar{X}=Z$.

Demostración. Supongamos que la función $F: Z \rightarrow Y$ es una extensión continua de la función perfecta $f$, donde $Z$ es un espacio de Hausdorff con las propiedades mencionadas. Sin pérdida de generalidad, podemos suponer que $Z=X \cup\{x\}$ con $x \notin X$. Dado que la fibra $f^{-1}(F(x))$ es un conjunto compacto y $x \notin f^{-1}(F(x))$, existen $U, V \subset Z$ abiertos y ajenos tales que $x \in U$ y $f^{-1}(F(x)) \subset V$. El conjunto $f(X-V)$ es cerrado en $Y$, por lo que $F^{-1}(f(X-V))$ es cerrado en $Z$. Por consiguiente

$$
\bar{X}-\bar{V} \subset \overline{X-V} \subset F^{-1}(f(X-V))=f^{-1} f(X-V) \subset X .
$$

Como $x \in \bar{X}-\bar{V}$, de lo anterior obtenemos la contradicción $x \in X$, es decir, $X=\bar{X}=Z$.

1.23. Teorema. Sea $f: X \rightarrow Y$ una función continua. Las siguientes condiciones son equivalentes

(i) $f$ es perfecta;

(ii) Para toda compactificación $\alpha Y$ de $Y$, la extensión $F_{\alpha}: \beta X \rightarrow \alpha Y$ de $f$ satisface $F_{\alpha}(\beta X-X) \subset \alpha Y-Y$;

(iii) La extensión $F: \beta X \rightarrow \beta Y$ de $f$ cumple $F(\beta X-X) \subset \beta Y-Y$;

(iv) Existe una compactificación $\alpha Y$ tal que la extensión $F_{\alpha}: \beta X \rightarrow \alpha Y$ de $f$ cumple con $F_{\alpha}(\beta X-X) \subset \alpha Y-Y$.

Demostración. Supongamos que la función $f: X \rightarrow Y$ es perfecta y tomemos la extensión $F_{\alpha}: \beta X \rightarrow \alpha Y$. Debido a que $f$ puede ser extendida sobre el conjunto $Z=F_{\alpha}^{-1}(Y)$, del lema anterior se sigue que $Z=X$, por lo tanto $F_{\alpha}(\beta X-X) \subset$ $\alpha Y-Y$, con lo que se establece (i) $\Longrightarrow$ (ii). Las implicaciones (ii) $\Longrightarrow$ (iii) y (iii) $\Longrightarrow$ (iv) son obvias. Como $F_{\alpha}$ es perfecta y $F_{\alpha}\left\lceil F_{\alpha}^{-1}(Y)=F_{\alpha}\lceil X=f\right.$, la prueba de (iv) $\Longrightarrow$ (i) la obtenemos de la proposición 1.21. 
1.24. Teorema. Si $f: X \rightarrow Y$ es una función perfecta y sobre, entonces el espacio $X$ es Čech completo si y sólo si $Y$ es Čech completo.

Demostración. Consideremos la extensión $F: \beta X \rightarrow \beta Y$ de $f$. Por el inciso (iii) del teorema anterior, la función $F$ verifica $F(\beta X-X) \subset \beta Y-Y$. Por lo tanto $F(\beta X-X)=\beta Y-Y$. Supongamos que $X$ es Čech completo. Luego, $\beta X-X=$ $\bigcup_{n \in \mathbb{N}} F_{n}$ donde $F_{n}$ es un subconjunto cerrado de $\beta X$ para todo $n \in \mathbb{N}$. Así que

$$
F(\beta X-X)=F\left(\bigcup_{n \in \mathbb{N}} F_{n}\right)=\bigcup_{n \in \mathbb{N}} F\left(F_{n}\right)=\beta Y-Y
$$

Dado que $F\left(F_{n}\right)$ es cerrado en $\beta Y$ para toda $n \in \mathbb{N}$, el espacio $Y$ es Čech completo. Para probar la suficiencia, sea $\beta Y-Y=\bigcup_{n \in \mathbb{N}} K_{n}$ donde $K_{n}$ es cerrado en $\beta Y$ para toda $n \in \mathbb{N}$. De aquí se desprende

$$
\beta X-X=F^{-1}(\beta Y-Y)=F^{-1}\left(\bigcup_{n \in \mathbb{N}} K_{n}\right)=\bigcup_{n \in \mathbb{N}} F^{-1}\left(K_{n}\right)
$$

donde $F^{-1}\left(K_{n}\right)$ es cerrado en $\beta X$. Por consiguiente, el espacio $X$ es Čech completo.

Sea $X$ un espacio de Tychonoff y $A \subset X$. La familia $\mathcal{B} \subset \mathcal{T}(X)$ recibe el nombre de base para $A$ en $X$ si todos los elementos de $\mathcal{B}$ contienen a $A$ y si $U$ es un conjunto abierto que contiene a $A$, entonces existe $B \in \mathcal{B}$ tal que $A \subset B \subset U$. El carácter de $A$ en $X$, denotado por $\chi(A, X)$, es el mínimo de todos los números cardinales $|\mathcal{B}|$, donde $\mathcal{B}$ es una base para $A$ en $X$.

Diremos que un espacio de Tychonoff $X$ es de tipo puntual numerable si para todo $x \in X$ existe un subespacio compacto $K \subset X$ tal que $x \in K$ y $\chi(K, X) \leq \omega$.

1.25. Proposición. Todo espacio Čech completo es de tipo puntual numerable.

Demostración. Sea $X$ un espacio Čech completo. Luego, $X=\bigcap_{n \in \mathbb{N}} G_{n}$ donde $G_{n} \in \mathcal{T}(\beta X)$. Tomemos cualquier $x \in X$. Existe $U_{1} \in \mathcal{T}(\beta X)$ tal que $x \in U_{1} \subset$ $\overline{U_{1}} \subset G_{1}$. Supongamos que tenemos la sucesión $\left\{U_{i}\right\}_{i \leq k} \subset \mathcal{T}(\beta X)$ para la cual $x \in U_{i} \subset \overline{U_{i}} \subset G_{i}$ con $i \leq k$. Consideremos $U_{k+1} \in \mathcal{T}(\beta X)$ que satisface $x \in U_{k+1} \subset$ $\overline{U_{k+1}} \subset U_{k} \cap G_{k+1}$. De esta forma, obtenemos una sucesión $\left\{U_{n}\right\}_{n \in \mathbb{N}} \subset \mathcal{T}(\beta X)$ tal que $x \in \bigcap_{n \in \mathbb{N}} U_{n}=\bigcap_{n \in \mathbb{N}} \overline{U_{n}} \subset \bigcap_{n \in \mathbb{N}} G_{n}=X$. Haciendo $K=\bigcap_{n \in \mathbb{N}} \overline{U_{n}}$ obtenemos lo pedido.

Un espacio $X$ de Tychonoff se llama casi $\check{C}$ ech completo si $X$ contiene un subespacio denso y Čech completo. En el teorema 1.33 probaremos que la casi Cech completitud se preserva bajo funciones abiertas; pero antes, introduzcamos el concepto de espacio de Baire y algunas de sus propiedades. 
Un espacio topológico $X$ es de Baire si la intersección de una familia numerable de subespacios abiertos y densos en $X$, es un subespacio denso en $X$.

Dado un espacio topológico $X$, se dice que el subconjunto $A \subset X$ es de primera categoría si $A=\bigcup_{n \in \mathbb{N}} A_{n} \operatorname{donde} \operatorname{int}\left(\overline{A_{n}}\right)=\emptyset$, para todo $n \in \mathbb{N}$. El subconjunto $A \subset X$ es de segunda categoría si $A$ no es de primera categoría.

1.26. Teorema. Un espacio $X$ es de Baire si y sólo si todo subconjunto abierto y no vacío de $X$ es de la segunda categoría.

Demostración. Sea $X$ un espacio de Baire. Tomemos $G \in \mathcal{T}^{*}(X)$. Supongamos que $G$ es de la primera categoría. Luego, $G=\bigcup_{n \in \mathbb{N}} G_{n} \operatorname{donde} \operatorname{int}\left(\overline{G_{n}}\right)=\emptyset$ para todo $n \in \mathbb{N}$. Debido a que $G \subset \bigcup_{n \in \mathbb{N}} \overline{G_{n}}$, obtenemos $X-G \supset \bigcap_{n \in \mathbb{N}}\left(X-\overline{G_{n}}\right)$. Por hipótesis, el conjunto $\bigcap_{n \in \mathbb{N}}\left(X-\overline{G_{n}}\right)$ es denso en $X$, ya que $X-\overline{G_{n}}$ lo es para todo $n \in \mathbb{N}$. Por lo tanto $X-G=\overline{X-G} \supset \overline{\bigcap_{n \in \mathbb{N}}\left(X-\overline{G_{n}}\right)}=X$. De aquí se desprende que $G=\emptyset$, lo cual es una contradicción.

Supongamos ahora que todo subconjunto abierto y no vacío de $X$ es de la segunda categoría. Tomemos una familia $\left\{U_{n}\right\}_{n \in \mathbb{N}} \subset \mathcal{T}(X)$, donde $\overline{U_{n}}=X$ para todo $n \in \mathbb{N}$. Sea $G \in \mathcal{T}^{*}(X)$. Probemos que $G \bigcap\left(\bigcap_{n \in \mathbb{N}} U_{n}\right) \neq \emptyset$. Puesto que cada abierto $U_{n}$ es denso en $X$, tenemos que $\operatorname{int}\left(\overline{X-U_{n}}\right)=\operatorname{int}\left(X-U_{n}\right)=\emptyset$. Por lo tanto, el conjunto $A=\bigcup_{n \in \mathbb{N}}\left(X-U_{n}\right)$ es de primera categoría. Tomando en cuenta que el abierto $G$ es de segunda categoría, concluimos que $G \cap(X-A) \neq \emptyset$, es decir, $G \bigcap\left(\bigcap_{n \in \mathbb{N}} U_{n}\right) \neq \emptyset$.

1.27. Teorema. Si $X$ es Čech completo entonces el espacio $X$ es de Baire.

Demostración. Supongamos que $X$ es un espacio Čech completo. Sea $\mathcal{U}=$ $\left\{\mathcal{U}_{n}\right\}_{n \in \mathbb{N}}$ una sucesión completa de cubiertas abiertas de $X$. Consideremos una familia $\left\{V_{n}\right\}_{n \in \mathbb{N}}$ de subconjuntos abiertos y densos de $X$. Sea $G \in \mathcal{T}^{*}(X)$. Probemos que $G \bigcap\left(\bigcap_{n \in \mathbb{N}} V_{n}\right) \neq \emptyset$. Puesto que $V_{1}$ es un subconjunto denso de $X$, tenemos que $G \cap V_{1} \neq \emptyset$. Escojamos $x \in G \cap V_{1}$. Existe $U_{1} \in \mathcal{U}_{1}$ tal que $x \in U_{1}$. Por regularidad, existe $G_{1} \in \mathcal{T}(x, X)$ para el cual $x \in G_{1} \subset \overline{G_{1}} \subset\left(V_{1} \cap G\right) \cap U_{1}$. Similarmente, como $G_{1} \cap V_{2} \neq \emptyset$ podemos encontrar un $G_{2} \in \mathcal{T}^{*}(X)$ tal que $G_{2} \subset \overline{G_{2}} \subset\left(V_{2} \cap G_{1}\right) \cap U_{2}$, donde $U_{2} \in \mathcal{U}_{2}$. Continuando con este procedimiento, obtenemos la sucesión decreciente $\left\{G_{n}\right\}_{n \in \mathbb{N}} \subset \mathcal{T}^{*}(X)$ que satisface

(a) $\cdots G_{2} \subset \overline{G_{2}} \subset G_{1} \subset \overline{G_{1}} \subset G$,

(b) $\overline{G_{n}} \subset U_{n} \in \mathcal{U}_{n}$ para todo $n \in \mathbb{N}, \mathrm{y}$

(c) $\overline{G_{n}} \subset G \cap\left(\bigcap_{i \leq n} V_{i}\right)$.

De $(a)$ y $(b)$ se desprende que la familia de subconjuntos cerrados $\mathcal{F}=\left\{\overline{G_{n}}\right\}_{n \in \mathbb{N}}$ es centrada y está dominada por la sucesión $\mathcal{U}$. Dado que $\mathcal{U}$ es completa, obtenemos que $\bigcap \mathcal{F}=\bigcap_{n \in \mathbb{N}} \overline{G_{n}} \neq \emptyset$. De aquí y de la propiedad $(c)$ resulta que $G \bigcap\left(\bigcap_{n \in \mathbb{N}} V_{n}\right) \neq \emptyset$. 
1.28. Proposición. Si $X=\bar{D}$ y $D$ es de Baire, entonces el espacio $X$ es de Baire.

Demostración. Sea $\left\{U_{n}\right\}_{n \in \mathbb{N}} \subset \mathcal{T}(X)$ donde $\overline{U_{n}}=X$ para cada $n \in \mathbb{N}$. Por demostrar que $\overline{\bigcap_{n \in \mathbb{N}} U_{n}}=X$. Para cada $n \in \mathbb{N}$ obtenemos que $\overline{D \cap U_{n}}=\overline{U_{n}}=$ $X=\bar{D}$. Por ser el subespacio $D$ de Baire, se desprende que $\overline{\bigcap_{n \in \mathbb{N}}\left(D \cap U_{n}\right)}=\bar{D}$. Por lo tanto $X=\bar{D}=\overline{\bigcap_{n \in \mathbb{N}}\left(D \cap U_{n}\right)} \subset \bar{D} \cap \overline{\bigcap_{n \in \mathbb{N}} U_{n}}=\overline{\bigcap_{n \in \mathbb{N}} U_{n}}$.

Aplicando el teorema 1.27 y la última proposición, obtenemos el siguiente corolario.

1.29. Corolario. Si $X$ es un espacio casi Čech completo, entonces $X$ es de Baire.

1.30. Teorema. Sea $f: X \rightarrow Y$ una función abierta, donde $X$ es de Baire. Entonces $Y$ es un espacio de Baire.

Demostración. Supongamos que $U_{n} \in \mathcal{T}(Y)$ es denso en $Y$ para todo $n \in \mathbb{N}$. Como $f$ es una función abierta, el conjunto $V_{n}=f^{-1}\left(U_{n}\right)$ es denso y abierto en $X$ para todo $n$. De modo que $G=\bigcap\left\{V_{n}: n \in \mathbb{N}\right\}$ es denso en $X$ y por lo tanto $H=f(G)$ es denso en $Y$. Observése finalmente que $H \subset \bigcap\left\{U_{n}: n \in \mathbb{N}\right\}$ y por lo tanto $\bigcap\left\{U_{n}: n \in \mathbb{N}\right\}$ es denso en $Y$.

Decimos que una familia $\mathcal{U} \subset \exp (X)$ es una casi cubierta de $X$ si $X=\overline{\cup \mathcal{U}}$ (ver [AL] y [Fr3]) En el caso en que $\mathcal{U} \subset \mathcal{T}(X)$ sea una casi cubierta de $X$, diremos que $\mathcal{U}$ es una casi cubierta abierta de $X$. Una sucesión $\left\{\mathcal{U}_{n}\right\}_{n \in \mathbb{N}}$ de casi cubiertas abiertas de $X$ se llamará abierta-completa si cualquier familia centrada $\mathcal{F} \subset \mathcal{T}(X)$ tal que $\mathcal{F} \cap \mathcal{U}_{n} \neq \emptyset$ para todo $n \in \mathbb{N}$, verifica $\bigcap \overline{\mathcal{F}} \neq \emptyset$.

1.31. Teorema. Un espacio $X$ es casi Čech completo si y sólo si $X$ posee una sucesión abierta-completa de casi cubiertas abiertas.

Demostración. Sea $G$ un subespacio Čech completo y denso en $X$. Tomemos una sucesión completa $\mathcal{G}=\left\{\mathcal{G}_{n}\right\}_{n \in \mathbb{N}}$ de cubiertas abiertas de $G$. Para cada $n \in \mathbb{N}$ hagamos $\mathcal{U}_{n}=\left\{U \in \mathcal{T}(X): U \cap G \in \mathcal{G}_{n}\right\}$. Verifiquemos que $\left\{\mathcal{U}_{n}\right\}_{n \in \mathbb{N}}$ es una sucesión abierta-completa de casi cubiertas abiertas de $X$. Sea $n \in \mathbb{N}$. Es evidente que $\mathcal{U}_{n} \subset \mathcal{T}(X)$. Consideremos un $H \in \mathcal{T}^{*}(X)$. Puesto que $G$ es denso y $G \subset \bigcup \mathcal{U}_{n}$, tenemos que $\emptyset \neq H \cap G \subset H \bigcap\left(\bigcup \mathcal{U}_{n}\right)$. De aquí concluimos que $H \cap U \neq \emptyset$ para algún $U \in \mathcal{U}_{n}$. Por lo tanto $X=\overline{\cup \mathcal{U}_{n}}$. Ahora probemos que la sucesión $\left\{\mathcal{U}_{n}\right\}_{n \in \mathbb{N}}$ es abierta-completa. Consideremos una familia centrada $\mathcal{F} \subset \mathcal{T}(X)$ tal que $\mathcal{F} \cap \mathcal{U}_{n} \neq \emptyset$ para todo $n \in \mathbb{N}$. Observemos que $\mathcal{F}_{G}=\{F \cap G: F \in \mathcal{F}\}$ es una familia centrada de subconjuntos de $G$. Sea $n \in \mathbb{N}$. Tomemos $F_{n} \in \mathcal{F} \cap \mathcal{U}_{n}$. Luego, $\left(F_{n} \cap G\right) \in \mathcal{G}_{n} \cap \mathcal{F}_{G}$. De la completitud de $\mathcal{G}$, obtenemos $\bigcap \overline{\mathcal{F}}_{G}{ }^{G} \neq \emptyset$. Debido a que $\emptyset \neq \bigcap{\overline{\mathcal{F}_{G}}}^{G} \subset \bigcap^{\mathcal{F}_{G}}=\bigcap_{F \in \mathcal{F}} \overline{F \cap G} \subset \bigcap^{\mathcal{F}}$, concluimos que la sucesión $\left\{\mathcal{U}_{n}\right\}_{n \in \mathbb{N}}$ es abierta-completa. 
Para probar la suficiencia, tomemos una sucesión abierta-completa $\mathcal{U}=\left\{\mathcal{U}_{n}\right\}_{n \in \mathbb{N}}$ de casi cubiertas abiertas de $X$ y demostremos que $G=\bigcap_{n \in \mathbb{N}}\left(\bigcup \mathcal{U}_{n}\right)$ es un subespacio denso y Čech completo de $X$.

Sea $n \in \mathbb{N}$. Consideremos la familia $\mathcal{U}_{n}^{\prime}=\left\{V \in \mathcal{T}(X): \bar{V} \subset U\right.$ para algún $\left.U \in \mathcal{U}_{n}\right\}$. Es inmediato que $\bigcup \mathcal{U}_{n}^{\prime}=\bigcup \mathcal{U}_{n}$ y $\mathcal{U}_{n}^{\prime n} \prec \mathcal{U}_{n}$. La familia $\mathcal{U}_{n}^{\prime \prime}=\mathcal{U}_{n}^{\prime} \cap G=\{V \cap G$ : $\left.V \in \mathcal{U}_{n}^{\prime}\right\} \subset \mathcal{T}(G)$ es una cubierta de $G$. En efecto, tomemos un $x \in G$. Luego, $x \in \bigcup \mathcal{U}_{n}^{\prime}$, de donde $x \in V$ para algún $V \in \mathcal{U}_{n}^{\prime}$. De modo que $\mathcal{U}_{n}^{\prime \prime}$ cubre a $G$. Probemos que la sucesión $\left\{\mathcal{U}_{n}^{\prime \prime}\right\}_{n \in \mathbb{N}}$ es completa. Sea $\mathcal{F} \subset \exp (G)$ una familia centrada tal que $\mathcal{F} \cap \mathcal{U}_{n}^{\prime \prime} \neq \emptyset$ para todo $n \in \mathbb{N}$. La colección $\mathcal{W}=\{W \in \mathcal{T}(X)$ : $W \supset F$ para algún $F \in \mathcal{F}\}$ es una familia centrada de subconjuntos abiertos de $X$. Notemos que si $F \in \mathcal{F} \cap \mathcal{U}_{n}^{\prime \prime}$ entonces $F \subset V \subset U$, donde $V \in \mathcal{U}_{n}^{\prime}$ y $U \in \mathcal{U}_{n}$. Luego, $\mathcal{W} \cap \mathcal{U}_{n} \neq \emptyset$ para todo $n \in \mathbb{N}$ y como $\mathcal{U}$ es abierta-completa, se desprende que $\bigcap \overline{\mathcal{W}} \neq \emptyset$. Tomemos un $x \in \bigcap \overline{\mathcal{W}}$ y verifiquemos que $x \in \bigcap \overline{\mathcal{F}}^{G}$. Si suponemos lo contrario, entonces existe un $F \in \mathcal{F}$ para el cual $x \notin \bar{F}^{G}=\bar{F} \cap G$. De $\mathcal{U}_{n}^{\prime} \cap \mathcal{W} \neq \emptyset$ se desprende que $x \in \overline{V_{n}}$ con $V_{n} \in \mathcal{U}_{n}^{\prime}$ y todo $n \in \mathbb{N}$. De aquí, y de la definición de $\mathcal{U}_{n}^{\prime}$, se sigue que $x \in \bigcap_{n \in \mathbb{N}}\left(\cup \mathcal{U}_{n}\right)=G$, y por lo tanto $x \notin \bar{F}$. Escojamos un $H \in \mathcal{T}(x, X)$ tal que $\bar{H} \cap F=\emptyset$. De aquí resulta que $X-\bar{H} \in \mathcal{W}$. Así que obtenemos la contradicción $x \in \overline{X-\bar{H}}$, y en consecuencia $x \in \bigcap^{G}$.

Para concluir con nuestra demostración, verifiquemos que $G$ es un subconjunto denso de $X$. Sea $U \in \mathcal{T}^{*}(X)$. Existe $U_{1} \in \mathcal{U}_{1}$ tal que $U_{1} \cap U \neq \emptyset$. Tomemos un $H_{1} \in \mathcal{T}^{*}(X)$ para el cual $H_{1} \subset \overline{H_{1}} \subset U_{1} \cap U$. Procediendo por inducción, obtenemos las familias de abiertos no vacíos $\mathcal{B}=\left\{U_{n}: U_{n} \in \mathcal{U}_{n}, n \in \mathbb{N}\right\}$ y $\mathcal{H}=\left\{H_{n}\right\}_{n \in \mathbb{N}}$ tales que $\overline{H_{n}} \subset U \bigcap\left(\bigcap_{i=1}^{n-1} H_{i}\right) \bigcap\left(\bigcap_{i=1}^{n} U_{i}\right)$. Como $\overline{H_{i+1}} \subset H_{i}$ y $H_{j_{*}} \subset\left(\bigcap_{i=1}^{n} U_{k_{i}}\right)$ donde $j_{*}=\max _{i \leq n} k_{i}$, la colección $\mathcal{F}=\mathcal{H} \cup \mathcal{B} \subset \mathcal{T}^{*}(X)$ es una familia centrada y verifica $\mathcal{F} \cap \mathcal{U}_{n} \neq \emptyset$ para todo $n \in \mathbb{N}$. De lo anterior, y por ser la sucesión $\mathcal{U}$ abierta-completa, obtenemos $\emptyset \neq \bigcap_{\mathcal{F}} \subset \bigcap_{n \in \mathbb{N}} H_{n}=\bigcap_{n \in \mathbb{N}} \overline{H_{n}}=H$. Pero $H \subset \bigcap_{n \in \mathbb{N}} U_{n} \subset G$, de donde se concluye que $U \cap G \neq \emptyset$.

1.32. Lema. Sea $\left\{\mathcal{U}_{n}\right\}_{n \in \mathbb{N}}$ una sucesión abierta-completa de casi cubiertas abiertas de $X$. Para cada $n \in \mathbb{N}$, tomemos $\mathcal{H}_{n}=\left\{H \in \mathcal{T}^{*}(X): H \subset U\right.$ con $\left.U \in \mathcal{U}_{n}\right\}$. Entonces $\left\{\mathcal{H}_{n}\right\}_{n \in \mathbb{N}}$ es una sucesión abierta-completa de casi cubiertas abiertas de $X$ y cada $\mathcal{H}_{n}$ es una $\pi$-base.

Demostración. Puesto que $\mathcal{U}_{n} \subset \mathcal{H}_{n}$, tenemos que $X=\overline{\bigcup \mathcal{H}_{n}}$ para todo $n \in \mathbb{N}$. Para verificar que $\left\{\mathcal{H}_{n}\right\}_{n \in \mathbb{N}}$ es abierta-completa, consideremos una familia centrada $\mathcal{F} \subset \mathcal{T}(X)$ tal que $\mathcal{F} \cap \mathcal{H}_{n} \neq \emptyset$ para todo $n \in \mathbb{N}$. Tomemos

$$
\mathcal{F}^{\prime}=\left\{U \text { : existe un } n \in \mathbb{N} \text { tal que } U \in \mathcal{U}_{n} \text { y } U \supset H \text { para algún } H \in \mathcal{F} \cap \mathcal{H}_{n}\right\} \text {. }
$$

La familia $\mathcal{F}^{\prime \prime}=\mathcal{F} \cup \mathcal{F}^{\prime}$ es centrada y verifica $\mathcal{F}^{\prime \prime} \cap \mathcal{U}_{n} \neq \emptyset$ para todo $n \in \mathbb{N}$. Debido a la casi Čech completitud de $X$, se sigue que $\bigcap^{\overline{\mathcal{F}^{\prime \prime}}} \neq \emptyset$. Tomando en cuenta la

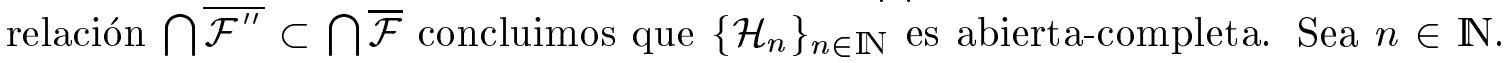


Tomemos un $G \in \mathcal{T}^{*}(X)$. Como $\mathcal{U}_{n}$ es casi cubierta, existe $U \in \mathcal{U}_{n}$ para el cual $G \cap U \neq \emptyset$. Por lo tanto $G \supset(G \cap U) \in \mathcal{H}_{n}$, de donde $\mathcal{H}_{n}$ es una $\pi$-base para todo $n \in \mathbb{N}$.

Recordemos $[\mathrm{Ku}]$ que dado un conjunto $(X, \leq)$ parcialmente ordenado, un subconjunto $A \subset X$ se llama cadena si $a \leq b$ ó $b \leq a$ para todos $a, b \in A$. El conjunto $(X, \leq)$ tiene cadenas acotadas, si para toda cadena $A \subset X$ existe un $x$ en $X$ tal que $a \leq x$ para cada $a \in A$. Un elemento $x_{0} \in X$ se llama maximal si, $x_{0} \leq y$ implica $x_{0}=y$ para todo $y \in X$. El Lema de Zorn nos afirma que todo conjunto parcialmente ordenado con cadenas acotadas tiene un elemento maximal.

1.33. Teorema. Sea $f: X \rightarrow Y$ una función abierta y sobre. Si $X$ es casi Čech completo, entonces $Y$ es un espacio casi Čech completo.

Demostración. En virtud del teorema 1.31, existe una sucesión abierta-completa $\left\{\mathcal{U}_{n}\right\}_{n \in \mathbb{N}}$ de casi cubiertas abiertas de $X$. Tomemos la sucesión $\left\{\mathcal{H}_{n}\right\}_{n \in \mathbb{N}}$ proporcionada por el lema anterior y construyamos una sucesión $\left\{\mathcal{H}_{n}^{\prime}\right\}_{n \in \mathbb{N}}$ tal que para todo $n \in \mathbb{N}$ se cumplan

(i) $\mathcal{H}_{n}^{\prime} \subset \mathcal{H}_{n}$;

(ii) $\mathcal{H}_{n+1}^{\prime}$ es un refinamiento de $\mathcal{H}_{n}^{\prime}$;

(iii) Si $H_{1}, H_{2} \in \mathcal{H}_{n}^{\prime}$ con $H_{1} \neq H_{2}$ entonces $f\left(H_{1}\right) \cap f\left(H_{2}\right)=\emptyset$;

(iv) la familia $f\left(\mathcal{H}_{n}^{\prime}\right)$ es ajena, y $Y=\overline{\bigcup f\left(\mathcal{H}_{n}^{\prime}\right)}$.

Sea $\mathcal{P}_{1}=\left\{\gamma \subset \mathcal{H}_{1}: f(\gamma)\right.$ es ajena $\}$. Proporcionemos un orden parcial $\leq$ en $\mathcal{P}_{1}$. Para cualesquiera $\gamma_{1}, \gamma_{2} \in \mathcal{P}_{1}$, diremos que $\gamma_{1} \leq \gamma_{2}$ si y sólo si $\gamma_{1} \subset \gamma_{2}$. Es claro que $\mathcal{P}_{1}$ tiene cadenas acotadas. Sea $\mathcal{H}_{1}^{\prime}$ un elemento maximal de $\mathcal{P}_{1}$. Verifiquemos que la familia $\mathcal{H}_{1}^{\prime}$ satisface (i)-(iv). La propiedad (ii) se sigue por vacuidad, la (i), (iii) y la primera parte de (iv), de la definición de $\mathcal{H}_{1}^{\prime}$. Probemos que $Y=\overline{\bigcup f\left(\mathcal{H}_{1}^{\prime}\right)}$ Si $G=Y-\overline{\bigcup f\left(\mathcal{H}_{1}^{\prime}\right)} \in \mathcal{T}^{*}(X)$, entonces $f^{-1}(G) \in \mathcal{T}^{*}(X)$. Por ser $\mathcal{H}_{1}$ una $\pi$-base, existe $U \in \mathcal{H}_{1}$ para el cual $U \subset f^{-1}(G)$. Consideremos $\mathcal{H}^{*}=\mathcal{H}_{1}^{\prime} \cup\{U\} \subset \mathcal{H}_{1}$. Dado que $f(U) \subset G$, la familia $f\left(\mathcal{H}^{*}\right)=f\left(\mathcal{H}_{1}^{\prime}\right) \cup\{f(U)\}$ es ajena. Por lo tanto, $\mathcal{H}^{*} \in \mathcal{P}_{1}$, contradiciendo de esta forma la maximalidad de $\mathcal{H}_{1}^{\prime}$.

Supongamos que tenemos $\mathcal{H}_{1}^{\prime}, \mathcal{H}_{2}^{\prime}, \ldots, \mathcal{H}_{n}^{\prime}$ que satisfacen las condiciones (i)-(iv). Para cada $U \in \mathcal{H}_{n}^{\prime}$, tomemos un elemento maximal $\mathcal{H}_{n+1}^{\prime}(U)$ de la familia

$$
\mathcal{P}_{n+1}=\left\{\gamma \subset \mathcal{H}_{n+1}: \bigcup \bar{\gamma} \subset U, \text { y } f(\gamma) \text { es ajena }\right\} .
$$

La familia $\mathcal{H}_{n+1}^{\prime}=\bigcup_{U \in \mathcal{H}_{n}^{\prime}} \mathcal{H}_{n+1}^{\prime}(U)$ cumple con las condiciones (i)-(iv). En efecto, la primera de ellas se cumple debido a que $\mathcal{H}_{n+1}^{\prime}(U) \subset \mathcal{H}_{n+1}$ para todo $U \in \mathcal{H}_{n}^{\prime}$. Dado $H \in \mathcal{H}_{n+1}$, existe $U \in \mathcal{H}_{n}^{\prime}$ tal que $H \subset U$, así que es válida (ii). La propiedad (iii) y la primera parte de la (iv) son satisfechas debido a lo siguiente. 
Sean $H_{1}, H_{2} \in \mathcal{H}_{n+1}^{\prime}$ con $H_{1} \neq H_{2}$. Luego existen $U_{1}, U_{2} \in \mathcal{H}_{n}^{\prime}$ para los cuales $H_{1} \subset U_{1}$ y $H_{2} \subset U_{2}$. Si $U_{1}=U_{2}=U$ entonces $H_{1}, H_{2} \in \mathcal{H}_{n+1}^{\prime}(U)$, y por lo tanto $f\left(H_{1}\right) \cap f\left(H_{2}\right)=\emptyset$. En caso contrario, tenemos que $f\left(U_{1}\right) \cap f\left(U_{2}\right)=\emptyset$ у como consecuencia $f\left(H_{1}\right) \cap f\left(H_{2}\right)=\emptyset$. Ahora verifiquemos que $Y=\overline{\bigcup f\left(\mathcal{H}_{n+1}^{\prime}\right)}$. Sea $U \in \mathcal{H}_{n}^{\prime}$. Por la maximalidad de $\mathcal{H}_{n+1}^{\prime}(U)$, obtenemos que $f(U) \subset \overline{U f\left(\mathcal{H}_{n+1}^{\prime}\right)}$. De aquí y de la definición de $\mathcal{H}_{n+1}^{\prime}$ se desprende que

$$
\begin{aligned}
\bigcup f\left(\mathcal{H}_{n}^{\prime}\right) & =\bigcup_{U \in \mathcal{H}_{n}^{\prime}} f(U) \subset \bigcup_{U \in \mathcal{H}_{n}^{\prime}} \overline{\bigcup f\left(\mathcal{H}_{n+1}^{\prime}(U)\right)} \subset \overline{\bigcup_{U \in \mathcal{H}_{n}^{\prime}} \bigcup f\left(\mathcal{H}_{n+1}^{\prime}(U)\right)}= \\
& =\overline{\bigcup f\left(\bigcup_{U \in \mathcal{H}_{n}^{\prime}} \mathcal{H}_{n+1}^{\prime}(U)\right)}=\overline{\bigcup f\left(\mathcal{H}_{n+1}^{\prime}\right) .}
\end{aligned}
$$

Por lo tanto $Y=\overline{\bigcup f\left(\mathcal{H}_{n}^{\prime}\right)} \subset \overline{\bigcup f\left(\mathcal{H}_{n+1}^{\prime}\right)}$, de donde $Y=\overline{\bigcup f\left(\mathcal{H}_{n+1}^{\prime}\right)}$.

Probemos que la sucesión de casi cubiertas abiertas $\left\{f\left(\mathcal{H}_{n}^{\prime}\right)\right\}_{n \in \mathbb{N}}$ de $Y$ es abiertacompleta. Sea $\mathcal{F} \subset \mathcal{T}(Y)$ una familia centrada tal que $\mathcal{F} \cap f\left(\mathcal{H}_{n}^{\prime}\right) \neq \emptyset$ para todo $n \in \mathbb{N}$. Para cada $n$ tomemos $F_{n} \in \mathcal{F} \cap f\left(\mathcal{H}_{n}^{\prime}\right)$. Luego $F_{n}=f\left(H_{n}\right)$ para algún $H_{n} \in \mathcal{H}_{n}^{\prime}$. Como $F_{n+1} \cap F_{n} \neq \emptyset$ se desprende que $H_{n+1} \subset H_{n}$. En efecto, como $H_{n+1} \in \mathcal{H}_{n+1}^{\prime}$, por la propiedad (ii) existe un $H^{\prime} \in \mathcal{H}_{n}^{\prime}$ tal que $H_{n+1} \subset H^{\prime}$. Si $H^{\prime} \neq H_{n}$ entonces $f\left(H^{\prime}\right) \cap f\left(H_{n}\right)=\emptyset$, lo cual contradice que $F_{n+1} \cap F_{n} \neq \emptyset$ y por lo tanto $H^{\prime}=H_{n}$. Como consecuencia de lo anterior, tenemos que la familia

$$
\mathcal{D}=\left\{f^{-1}(F) \cap H_{n}: F \in \mathcal{F}, n \in \mathbb{N}\right\} \subset \mathcal{T}^{*}(X)
$$

es centrada. En efecto, consideremos $\left(f^{-1}\left(G_{n_{1}}\right) \cap H_{n_{1}}\right) \cap\left(f^{-1}\left(G_{n_{2}}\right) \cap H_{n_{2}}\right) \cap \cdots \cap$ $\left(f^{-1}\left(G_{n_{p}}\right) \cap H_{n_{p}}\right)=f^{-1}\left(\bigcap_{j=1}^{p} G_{n_{j}}\right) \cap H_{n_{k}} \operatorname{con} p \in \mathbb{N}, G_{n_{j}} \in \mathcal{F}$ y $n_{k}=\max \left\{n_{j}\right\}_{j \leq p}$. Para cada $n_{j}$ escojamos $F_{n_{j}} \in \mathcal{F} \cap f\left(\mathcal{H}_{n_{j}}^{\prime}\right)$, así que $G=G_{n_{1}} \cap G_{n_{2}} \cap \cdots \cap G_{n_{p}} \cap$ $F_{n_{1}} \cap F_{n_{2}} \cap \cdots \cap F_{n_{p}}=\bigcap_{j=1}^{p} G_{n_{j}} \cap F_{n_{k}} \neq \emptyset$.

Tomemos un $y \in G$, luego $y=f\left(h_{n_{k}}\right)$ para algún $h_{n_{k}} \in H_{n_{k}}$ y como $y \in \bigcap_{j=1}^{p} G_{n_{j}}$ obtenemos que $h_{n_{k}} \in f^{-1}\left(\bigcap_{j=1}^{p} G_{n_{j}}\right) \cap H_{n_{k}}$, es decir, la familia $\mathcal{D}$ es centrada.

De $H_{n} \subset f^{-1}\left(F_{n}\right)$ y por (i) obtenemos que $H_{n} \in \mathcal{D} \cap \mathcal{H}_{n}$ para todo $n$ y por ser $\left\{\mathcal{H}_{n}\right\}_{n \in \mathbb{N}}$ abierta-completa, existe un $x \in \cap \overline{\mathcal{D}}^{X}$. Por lo tanto, para todo $F \in \mathcal{F}$ tenemos que $x \in \overline{\left(f^{-1}(F) \cap H_{1}\right)}$, y en consecuencia $f(x) \in \overline{f\left(f^{-1}(F) \cap H_{1}\right)} \subset \bar{F}$. De aquí concluimos que la sucesión $\left\{f\left(\mathcal{H}_{n}^{\prime}\right)\right\}_{n \in \mathbb{N}}$ es abierta-completa. En virtud del Teorema 1.31, el espacio $Y$ es casi Čech completo.

1.34. Ejemplo. [CČN] Existe un espacio no Čech completo que posee una cubierta abierta y puntualmente finita de subespacios Čech completos.

Demostración. Sean los conjuntos $\mathcal{C}=\left\{f: \mathbb{N} \rightarrow \omega_{1}: f\right.$ es creciente $\}$ y $\mathcal{Y}=\{\delta \subset$ $\mathcal{C}:|f(\mathbb{N}) \cap g(\mathbb{N})|<\omega$ para $f, g \in \delta$ con $f \neq g\}$. Introduzcamos un orden parcial 
en $\mathcal{Y}$. Para cualesquiera $\delta_{1}, \delta_{2} \in \mathcal{Y}$, diremos que $\delta_{1} \leq \delta_{2}$ si y sólo si $\delta_{1} \subset \delta_{2}$. Es evidente que $\leq$ es un orden parcial en $\mathcal{Y}$. Afirmamos que cada cadena es acotada. En efecto, sea $C \subset \mathcal{Y}$ una cadena. Probemos que existe $\delta \in \mathcal{Y}$ tal que $\gamma \leq \delta$ para todo $\gamma \in C$. Sea $\delta=\cup C$. Es claro que $\gamma \leq \delta$ para cualquier $\gamma \in C$ y también lo es el hecho que $\delta \subset \mathcal{C}$. Sean $f, g \in \delta$ con $f \neq g$. Luego, $f \in \gamma_{1}$ y $g \in \gamma_{2}$ para determinados $\gamma_{1}, \gamma_{2} \in C$. Como $C$ es cadena, podemos suponer que $\gamma_{1} \subset \gamma_{2}$, de donde $f, g \in \gamma_{2}$. Por lo tanto, el conjunto $f(\mathbb{N}) \cap g(\mathbb{N})$ es finito. De lo anterior se desprende que $\delta \in \mathcal{Y}$.

Sea $Y$ un elemento maximal de $\mathcal{Y}$. Tomemos el conjunto $X=Y \cup V$ donde

$$
V=\omega_{1} \times[Y]^{<\omega}=\left\{(\alpha, \phi): \alpha \in \omega_{1} \text { y } \phi \text { es un subconjunto finito de } Y\right\} .
$$

Dados $y \in Y$ y $n \in \mathbb{N}$ consideremos los conjuntos

$$
\begin{aligned}
D(y, n) & =y(\{m \in \mathbb{N}: m \geq n\}) \subset \omega_{1}, \\
U(y, n) & =\{y\} \cup\{(\alpha, \phi) \in X: y \in \phi \text { y } \alpha \in D(y, n)\}, \\
\mathcal{U}_{n} & =\{U(y, n): y \in Y\} \cup\{\{v\}: v \in V\} .
\end{aligned}
$$

Dado $y \in Y$, hagamos $\mathcal{B}_{y}=\{U(y, n): n \in \mathbb{N}\}$. Si $y \in V$ hacemos $\mathcal{B}_{y}=\{\{y\}\}$. Es fácil ver que las familias $\left\{\mathcal{B}_{x}: x \in X\right\}$ generan una única topología $\tau$ como bases locales.

Observemos que para cada $n \in \mathbb{N}$, la cubierta abierta $\mathcal{U}_{n}$ es puntualmente finita y está constituida de subespacios Čech completos. En efecto, sea $x \in X$. Verifiquemos que $\left|\left\{U \in \mathcal{U}_{n}: x \in U\right\}\right|<\omega$. Consideremos el caso cuando $x \in V$. Luego, $x=\left(\alpha, \phi_{0}\right)$ con $\alpha \in \omega_{1}$ y $\phi_{0}$ un subconjunto finito de $Y$. De la definición de $U(y, n)$ se sigue que $x \notin U(y, n)$ si $y \notin \phi_{0}$. Por lo tanto $\left\{U \in \mathcal{U}_{n}: x \in U\right\} \subset$ $\left\{U(y, n): y \in \phi_{0}\right\} \cup\{\{x\}\}$. Ahora bien, si $x \in Y$, entonces $x=y$ para alguna función $y \in Y$. De modo que $\left\{U \in \mathcal{U}_{n}: x \in U\right\}=\{U(y, n)\}$. En ambas posibilidades se obtiene que $\left|\left\{U \in \mathcal{U}_{n}: x \in U\right\}\right|<\omega$. Para demostrar que los elementos de $\mathcal{U}_{n}$ son subespacios Cech completos de $X$, basta con probarlo para cada vecindad $U(y, n)$. Notemos que todos los puntos de $U(y, n)$ son aislados, excepto la función $y$. La familia $\left\{B_{k}\right\}_{k \in \mathbb{N}} \subset U(y, n)$, donde $B_{k}=\{y\} \cup\left\{\left(\alpha_{k}, \phi\right) \in X: y \in \phi\right.$ y $\alpha_{k}=$ $y(n+k)\}$, es base en $y$. Por lo tanto, la Čech completitud de $U(y, n)$ se sigue del teorema 1.18 .

Probemos que $X$ no es Čech completo. Supongamos lo contrario. Sea $\left\{\lambda_{n}\right\}_{n \in \mathbb{N}}$ una sucesión completa de cubiertas abiertas de $X$ tal que $\lambda_{n} \subset \mathcal{B}$ para todo $n \in \mathbb{N}$ (proposición1.17). Sin perder la generalidad, podemos suponer que $\lambda_{n} \subset \bigcup_{k=n}^{\infty} \mathcal{U}_{k}$. En efecto, para cada $n \in \mathbb{N}$ sea $\lambda_{n}^{\prime}=\lambda_{n} \wedge \mathcal{B}_{n}=\left\{U \cap V: U \in \lambda_{n}, V \in \mathcal{B}_{n}\right\}$, donde $\mathcal{B}_{n}=\bigcup_{k=n}^{\infty} \mathcal{U}_{k}$. Es inmediato que $X=\bigcup \lambda_{n}^{\prime}$ y $\lambda_{n}^{\prime} \subset \mathcal{T}(X)$ para toda $n \in \mathbb{N}$. Afirmamos que la sucesión $\lambda^{\prime}=\left\{\lambda_{n}^{\prime}\right\}_{n \in \mathbb{N}}$ de cubiertas abiertas de $X$ es completa. Sea $\mathcal{F} \subset \exp (X)$ una familia centrada. Supóngase que existen $\left\{F_{n}\right\} \subset \mathcal{F}$ y $\left\{W_{n}\right\} \subset$ 
$\lambda^{\prime}$ con $W_{n} \in \lambda_{n}^{\prime}$, tales que $F_{n} \subset W_{n}$ para todo $n \in \mathbb{N}$. Como $\lambda_{n}^{\prime} \subset \lambda_{n}$, obtenemos que $F_{n} \subset U_{n}$ para algún $U_{n} \in \lambda_{n}$ y todo $n \in \mathbb{N}$. Debido a que la sucesión $\left\{\lambda_{n}\right\}_{n \in \mathbb{N}}$ es completa, concluimos que $\bigcap \overline{\mathcal{F}} \neq \emptyset$. Haciendo $\lambda_{n}=\lambda_{n}^{\prime}$ para todo $n$, obtenemos lo deseado.

Para cada $n \in \mathbb{N}$ consideremos el conjunto $\mathcal{D}_{n}=\left\{D(y, m): U(y, m) \in \lambda_{n}, y \in Y\right\}$. Sea $D=\bigcap_{n \in \mathbb{N}}^{\infty} \cup \mathcal{D}_{n} \subset \omega_{1}$. Afirmamos que $D \neq \emptyset$. Si $D=\emptyset$ entonces $\omega_{1}=$ $\bigcup_{n \in \mathbb{N}}\left(\omega_{1}-\bigcup \mathcal{D}_{n}\right)$. Existe $n_{0} \in \mathbb{N}$ para el cual $\left|\omega_{1}-\cup \mathcal{D}_{n_{0}}\right|=\omega_{1}$. Luego, existe una función creciente $z: \mathbb{N} \rightarrow \omega_{1}$ tal que $z(\mathbb{N}) \cap\left(\cup \mathcal{D}_{n_{0}}\right)=\emptyset$. Por la maximalidad de $Y$, elegimos $y \in Y$ tal que $|y(\mathbb{N}) \cap z(\mathbb{N})|=\omega$. Probemos que $y \notin \bigcup \lambda_{n_{0}}$, lo que contradice que $\lambda_{n_{0}}$ sea cubierta. Si $y \in \bigcup \lambda_{n_{0}}$ entonces $y \in U(y, m)$ para algún $m \geq n_{0}$. Dado que $U(y, m) \in \lambda_{n_{0}}$ se sigue que $D(y, m) \in \mathcal{D}_{n_{0}}$. Luego, $D(y, m) \cap z(\mathbb{N})=\emptyset$. Así que $|y(\mathbb{N}) \cap z(\mathbb{N})|<\omega$, lo cual es contradictorio con la elección de $y$. Por lo tanto $D \neq \emptyset$.

Sea $\beta \in D$. Para cada $k \in \mathbb{N}$ existen $y_{k} \in Y$ y $n_{k} \geq k$ tales que $\beta \in D\left(y_{k}, n_{k}\right)$ y $U\left(y_{k}, n_{k}\right)=H_{k} \in \lambda_{k}$. Notemos que la sucesión $\left\{y_{k}\right\}_{k \in \mathbb{N}}$ es infinita. De lo contrario, existe $k_{0} \in \mathbb{N}$ tal que $y=y_{k}$ para todo $k \geq k_{0}$. De aquí resulta que $\beta \in$ $D(y, m)$ para todo $m \geq \max \left\{n_{1}, n_{2}, \ldots, n_{k_{0}}\right\}$ lo que contradice que $\bigcap_{n \in \mathbb{N}} D(y, n)=$ $\emptyset$. Consideremos la familia centrada de cerrados $\left\{F_{n}\right\}_{n \in \mathbb{N}}$ donde $F_{n}=\left\{\left(\beta, \phi_{j}\right): j \geq\right.$ $n\}$ y $\phi_{j}=\left\{y_{i}\right\}_{i \leq j}$. Es claro que $F_{n} \subset H_{n}$ para todo $n$. Puesto que $\bigcap_{n \in \mathbb{N}} F_{n}=\emptyset$, se contradice la completitud de $\left\{\lambda_{n}\right\}_{n \in \mathbb{N}}$.

1.35. Lema. Sea $f: X \rightarrow Y$ una función abierta y sobre, donde $Y$ es un espacio paracompacto y $X$ un espacio de Tychonoff. Consideremos un subespacio abierto $G$ de $Z=F^{-1}(Y)$ para el cual $f(X \cap G)=Y$ donde $F$ es la extensión continua de $f$ sobre $\beta X$ y $\beta Y$. Entonces existe $H \in \mathcal{T}^{*}(Z)$ tal que $f(X \cap H)=Y$ y cl $(H) \subset G$.

Demostración. Usaremos la convención $\operatorname{cl}_{Z}(G)=\bar{G}$ para todo $G \subset Z$. Fijémos $y \in Y$. Sea $z_{y} \in f^{-1}(y) \cap X \cap G$. Por regularidad, existe $V(y) \in \mathcal{T}(Z)$ para el cual $z_{y} \in V(y) \subset \overline{V(y)} \subset G$.

Observemos que la colección $\mathcal{V}=\{f(X \cap V(y)): y \in Y\}$ es una cubierta abierta de $Y$. En efecto, como $y \in Y$ y $z_{y} \in V(y) \subset \overline{V(y)} \subset G$ vemos que $y=f\left(z_{y}\right) \in$ $f(X \cap V(y))$. Puesto que el espacio $Y$ es paracompacto, existe un refinamiento abierto localmente finito $\mathcal{U}=\left\{U_{s}: s \in S\right\}$ de $\mathcal{V}$. Para cada $s \in S$ fijemos $y(s) \in Y$ tal que $U_{s} \subset f(X \cap V(y(s))$ y consideremos el conjunto

$$
H=\bigcup_{s \in S}\left(V(y(s)) \bigcap g^{-1}\left(U_{s}\right)\right)
$$

Probemos que $H$ es el conjunto deseado. Es evidente que $H \in \mathcal{T}^{*}(Z)$. Para cerciorarnos de la igualdad $f(X \cap H)=Y$, tomemos $y \in Y$. Luego $y \in U_{s} \subset$ $f(X \cap V(y(s))$ para algún $s \in S$. De modo que $y=f(z)$ para algún $z \in X \cap V(y(s))$. 
Como $z \in X \cap V(y(s)) \cap f^{-1}(y)$ obtenemos que $z \in X \cap V(y(s)) \cap g^{-1}\left(U_{s}\right) \subset X \cap H$, y por lo tanto $Y \subset f(X \cap H)$.

Finalmente, obtengamos la contención $\bar{H} \subset G$. Nótese que $\left\{g^{-1}\left(U_{s}\right): s \in S\right\}$ es una familia abierta localmente finita de $Z$, pues $\left\{U_{s}: s \in S\right\}$ lo es de $Y$, y por lo tanto también lo es la familia $\left\{V(y(s)) \cap g^{-1}\left(U_{s}\right): s \in S\right\}$. De ésto se desprende que

$$
\bar{H}=\bigcup_{s \in S} \overline{\left(V(y(s)) \bigcap g^{-1}\left(U_{s}\right)\right)} \subset \bigcup_{s \in S} \overline{V(y(s))} \subset G .
$$

1.36. Teorema (Pasynkov). Sea $f: X \rightarrow Y$ una función abierta y sobre, donde el espacio $X$ es Čech completo y $Y$ es paracompacto. Entonces, existe $A \subset X$ cerrado y $G_{\delta}$ tal que la restricción $f\lceil A: A \rightarrow Y$ es una función perfecta y sobre.

Demostración. Consideremos la extensión $F: \beta X \rightarrow \beta Y$ de la función $f$ y la función perfecta $g=F_{Y}: Z \rightarrow Y$ donde $Z=F^{-1}(Y)$. Al igual que en la demostración del lema anterior, cuando explícitamente no se indique otra cosa, dado cualquier $G \subset Z$, usaremos la convención $\operatorname{cl}_{Z}(G)=\bar{G}$. Como $X$ es Čech completo y $Z \subset \beta X$, podemos expresar al espacio $X$ de la forma $X=\bigcap_{i \in \mathbb{N}} G_{i}$ donde $G_{i} \in \mathcal{T}^{*}(Z)$ para todo $i \in \mathbb{N}$. Ahora, definamos inductivamente una sucesión $\left\{H_{i}\right\}_{i \in \omega} \subset \mathcal{T}^{*}(Z)$ donde $H_{0}=Z$, tal que para todo $i \in \mathbb{N}$ se cumplan las siguientes condiciones

(i) $\overline{H_{i}} \subset G_{i} \cap H_{i-1} \mathrm{y}$

(ii) $f\left(X \cap H_{i}\right)=Y$.

Supongamos que hemos construido $H_{i-1}$ que satisface (i) y (ii). Dado que el conjunto $G=G_{i} \cap H_{i-1}$ es un abierto no vacío en $Z$ y se cumple la igualdad

$$
Y=f\left(X \cap H_{i-1}\right)=f\left(X \cap G_{i} \cap H_{i-1}\right)=f(X \cap G),
$$

el lema anterior nos proporciona un $H_{i} \in \mathcal{T}^{*}(Z)$ con $f\left(X \cap H_{i}\right)=Y$ y $\overline{H_{i}} \subset$ $G_{i} \cap H_{i-1}$. De las contenciones

$$
H_{i} \subset \overline{H_{i}} \subset G_{i} \cap H_{i-1} \subset H_{i-1}
$$

obtenemos $\bigcap_{i \in \mathbb{N}} H_{i} \subset \bigcap_{i \in \mathbb{N}} \overline{H_{i}} \subset \bigcap_{i \in \mathbb{N}} H_{i}$, de donde $\bigcap_{i \in \mathbb{N}} H_{i}=\bigcap_{i \in \mathbb{N}} \overline{H_{i}}$. Chequemos que el conjunto $A=\bigcap_{i \in \mathbb{N}} H_{i}=\bigcap_{i \in \mathbb{N}} \overline{H_{i}}$ satisface la conclusión del teorema.

Tomando en cuenta que $H_{i} \subset G_{i}$ para toda $i \in \mathbb{N}$, obtenemos que $A$ es un subconjunto cerrado y $G_{\delta}$ de $X$.

La restricción $f\lceil A$ es una función perfecta, debido a que $g\lceil A$ es perfecta y a la igualdad $f\lceil A=g\lceil A$, pues $A \subset X \subset Z$. 
Finalmente, deduzcamos la igualdad $f(A)=Y$. Sea $y \in Y$. De (ii) se desprende que $f^{-1}(y) \cap \overline{H_{i}} \neq \emptyset$ para todo $i \in \mathbb{N}$, y por $(*)$ la familia $\left\{f^{-1}(y) \cap \overline{H_{i}}: i \in \mathbb{N}\right\}$ es centrada en $f^{-1}(y)$. Por lo tanto

$$
\emptyset \neq \bigcap_{i \in \mathbb{N}}\left(f^{-1}(y) \bigcap \overline{H_{i}}\right)=f^{-1}(y) \bigcap\left(\bigcap_{i \in \mathbb{N}} \overline{H_{i}}\right)=f^{-1}(y) \bigcap A
$$

lo cual implica que $y \in f(A)$.

\section{El espacio $\beta \omega$}

Consideremos $\omega$ con la topología discreta.

Sea $\beta \omega=\omega \cup\{\xi: \xi$ es ultrafiltro en $\omega$ con $\bigcap \xi=\emptyset\}$ y $\omega^{*}=\beta \omega-\omega$. Definiremos una topología en el conjunto $\beta \omega$.

Para cualquier $U \subset \omega$, consideremos $[U]=\left\{\xi \in \omega^{*}: U \in \xi\right\}$ y $\widehat{U}=U \cup[U]$.

1.37. Lema. Para cualesquiera $U, V \subset \omega$ se cumplen las siguientes propiedades

(a) $\widehat{U \cap V}=\widehat{U} \cap \widehat{V}$.

(b) $\widehat{U \cup V}=\widehat{U} \cup \widehat{V}$.

(c) $\widehat{\omega-U}=\beta \omega-\widehat{U}$.

Demostración. Para verificar (a) y (b), basta probar las igualdades (a') $[U \cap$ $V]=[U] \cap[V]$ y $\left(b^{\prime}\right)[U \cup V]=[U] \cup[V]$. Estas propiedades se obtienen de las correspondientes equivalencias

$\xi \in[U \cap V] \Longleftrightarrow U \cap V \in \xi \Longleftrightarrow U \in \xi \mathrm{y} V \in \xi \Longleftrightarrow \xi \in[U] \cap[V] \mathrm{y}$

$\xi \in[U \cup V] \Longleftrightarrow U \cup V \in \xi \Longleftrightarrow U \in \xi \circ V \in \xi \Longleftrightarrow \xi \in[U] \cup[V]$.

De forma análoga, para (c) es suficiente con verificar $[\omega-U]=\omega^{*}-[U]$, la cual se sigue de $\xi \in[\omega-U] \Longleftrightarrow \omega-U \in \xi \Longleftrightarrow U \notin \xi \Longleftrightarrow \xi \in \omega^{*}-[U]$.

Nótese que también es válida la igualdad $\beta \omega=\bigcup_{U \subset \omega} \widehat{U}$. De aquí y por la propiedad (a), consideremos la única topología $\tau$ en $\beta \omega$ tal que la familia $\{\widehat{U}\}_{U \subset \omega}$ es base para $\tau$. El siguiente teorema proporciona algunas de las propiedades básicas de $\beta \omega$.

1.38. Teorema. El espacio $\beta \omega$ satisface lo siguiente:

(i) $\omega$ es denso en $\beta \omega$ y los elementos de $\omega$ son los únicos puntos aislados de $\beta \omega$;

(ii) $\beta \omega$ es un espacio compacto;

(iii) $\widehat{U}=\bar{U}$ para todo $U \subset \omega$;

(iv) Los conjuntos de la forma $[U]$ con $U \subset \omega$, son los conjuntos abiertos-cerrados de $\omega^{*}$; 
(v) Si $A, B \subset \omega$ con $A \cap B=\emptyset$, entonces $\bar{A} \cap \bar{B}=\emptyset$;

(vi) Si $G \in \mathcal{T}(\beta \omega)$ entonces $\bar{G} \in \mathcal{T}(\beta \omega)$.

Demostración. Sea $\widehat{U} \in \mathcal{T}^{*}(\beta \omega)$. Luego, $\widehat{U} \cap \omega=U$, de donde $\bar{\omega}=\beta \omega$. Si $x \in \omega$, entonces $\{x\}=\widehat{\{x\}}$, así que $\{x\} \in \mathcal{T}(\beta \omega)$. Recíprocamente, si $\{x\} \in \mathcal{T}(\beta \omega)$ entonces $\{x\} \cap \omega \neq \emptyset$. Por lo tanto $x \in \omega, \mathrm{y}$ (i) queda probado.

Para verificar (ii), probemos primero que $\beta \omega$ es de Hausdorff. Sean $x, y \in \beta \omega$ con $x \neq y$. Debido al inciso anterior, basta considerar los casos $(a) x, y \in \omega^{*} \mathrm{y}$ (b) $x \in \omega, y \in \omega^{*}$. Para la primera posibilidad, sea $U \in x-y$. Luego, $\omega-U \in y$. Por lo tanto, los abiertos ajenos $\widehat{U}$ y $(\widehat{\omega-U})$ contienen a $x$ y a $y$ respectivamente. En el caso $(b)$, tenemos que $\{x\} \notin y$. Así que $x \in\{x\}, y \in(\omega \widehat{-\{x\}})$ y los abiertos $\{x\},(\omega-\widehat{-\{} x\})$ son ajenos.

Para probar que $\beta \omega$ es compacto, tomemos una familia centrada de cerrados $\left\{F_{s}\right\}_{s \in S}$ en $\beta \omega$. Para cada $s \in S$ se cumple que $\beta \omega-F_{s}=\bigcup_{t \in T_{s}} \widehat{U_{t}}$. Por lo tanto

$$
F_{s}=\bigcap_{t \in T_{s}}\left(\beta \omega-\widehat{U_{t}}\right)=\bigcap_{t \in T_{s}}\left(\widehat{\omega-U_{t}}\right) \operatorname{con} s \in S
$$

Notemos que $\left\{\left(\widehat{\omega-U_{t}}\right)\right\}_{t \in \cup_{s} \in S T_{s}}$ es centrada, pues

$\left(\omega-\widehat{U}_{t_{1}}\right) \cap\left(\omega-\widehat{U}_{t_{2}}\right) \cap \cdots \cap\left(\omega \widehat{-U}_{t_{k}}\right)=\left(\beta \omega-{\widehat{U_{t_{1}}}}\right) \cap\left(\beta \omega-{\widehat{U_{t_{2}}}}\right) \cap \cdots \cap\left(\beta \omega-{\widehat{U_{t_{k}}}}\right) \supset$ $F_{s_{1}} \cap F_{s_{2}} \cap \cdots \cap F_{s_{k}} \neq \emptyset$. En la igualdad hemos usado la parte $(c)$ del lema 1.37. De aquí, la familia $\left\{\left(\omega-U_{t}\right)\right\}_{t \in \cup_{s \in S} T_{s}}$ es centrada. Sea $\xi$ un ultrafiltro en $\omega$ para el cual $\left\{\left(\omega-U_{t}\right)\right\}_{t \in \cup_{s} \in S T_{s}} \subset \xi$. Si $\bigcap \xi \neq \emptyset$ entonces existe $x \in \omega$ tal que $\xi=\{A \subset \omega: x \in$ $A\}$. De aquí concluimos que $x \in \bigcap_{\substack{t \in \cup T_{s} \\ s \in S}}\left(\omega-U_{t}\right) \subset \bigcap_{\substack{t \in \cup T_{s} \\ s \in S}}\left(\widehat{\omega-U_{t}}\right)=\bigcap_{s \in S} F_{s}$. Para el caso $\bigcap \xi=\emptyset$, tenemos que $\xi \in \bigcap_{\substack{t \in \cup T_{s} \\ s \in S}}\left(\widehat{\omega-U_{t}}\right)=\bigcap_{s \in S} F_{s}$. En ambos casos obtenemos que $\bigcap_{s \in S} F_{s} \neq \emptyset$. Por lo tanto, el espacio $\beta \omega$ es compacto.

Probemos (iii). Sea $x \in \bar{A}$. Dado que $A \subset \widehat{A}$, el caso $x \in A$ es claro. Luego, podemos suponer que $x \in \bar{A}-A$. Si $x \notin \widehat{A}$ entonces $A \notin x$. De aquí que $\omega-A \in x$, es decir, $x \in[\omega-A]$. Por lo tanto $x \in(\widehat{\omega-A})$. Como $(\widehat{\omega-A}) \cap A=\emptyset$, se contradice que $x \in \bar{A}$.

Para verificar $\widehat{A} \subset \bar{A}$, tomemos $x \in \widehat{A}$ y un elemento básico $\widehat{U} \in \mathcal{T}(\beta \omega, x)$. El caso no trivial es cuando $x \in \widehat{A}-A$. De modo que podemos suponer que $x \in[A]$, o bien, $A \in x$. Dado que $U \in x$ y $A \in x$, se desprende que $A \cap U \neq \emptyset$. Por lo tanto $\widehat{U} \cap A \neq \emptyset$.

Verifiquemos (iv). Dado que $\omega$ es un subconjunto abierto de $\beta \omega$, el residuo $\omega^{*}=$ $\beta \omega-\omega$ es cerrado y por lo tanto un subespacio compacto de $\beta \omega$. Por el inciso anterior, todo conjunto $[U]$ es abierto y cerrado en $\omega^{*}$. Ahora supongamos que $W$ 
es un subconjunto abierto y cerrado de $\omega^{*}$. La familia $\mathcal{A}=\{[U]: U \subset \omega$ y $[U] \subset W\}$ es una cubierta abierta de $W$. Como $W$ es un subconjunto cerrado de $\omega^{*}$, se sigue que es compacto. Existe $\left\{\left[U_{i}\right]: U_{i} \subset \omega,\left[U_{i}\right] \subset W, i=1,2, \ldots, k\right\} \subset \mathcal{A}$ tal que $W=\bigcup_{i=1}^{k}\left[U_{i}\right]$. Del lema 1.37 (b) se desprende que $W=\left[\bigcup_{i=1}^{k} U_{i}\right]$, es decir tiene la forma deseada.

Para la propiedad (v) se sigue del lema 1.37 (a) y del inciso (iii) que $\bar{A} \cap \bar{B}=$ $\overline{A \cap B}=\bar{\emptyset}=\emptyset$.

Sea $G \in \mathcal{T}(\beta \omega)$. Por ser $\omega$ un subespacio denso de $\beta \omega$, obtenemos que $\bar{G}=\overline{G \cap \omega}$. De (iii) se desprende que $\bar{G}=\widehat{G}$, con lo que queda establecido (vi).

De acuerdo al teorema 1.4, el siguiente resultado nos garantiza que $\beta \omega$ es la compactificación de Stone-Čech del espacio discreto $\omega$.

1.39. Teorema. Sea $f: \omega \rightarrow Z$ una función continua, donde $Z$ un espacio compacto. Existe una función $F: \beta \omega \rightarrow Z$ continua tal que $F\lceil\omega=f$.

Demostración. Sean $B_{1}$ y $B_{2}$ subconjuntos cerrados y ajenos de $Z$. De acuerdo a la proposición 1.2, es suficiente demostrar que $\overline{f^{-1}\left(B_{1}\right)} \cap \overline{f^{-1}\left(B_{2}\right)}=\emptyset$. Como $f^{-1}\left(B_{1}\right)$ y $f^{-1}\left(B_{2}\right)$ son subconjuntos ajenos de $\omega$, del teorema $1.38(\mathrm{v})$ obtenemos que $\overline{f^{-1}\left(B_{1}\right)} \cap \overline{f^{-1}\left(B_{2}\right)}=\emptyset$.

1.40. Teorema. El espacio $\beta \omega$ satisface $|\beta \omega|=2^{\mathfrak{c}}$ y $w(\beta \omega)=\mathfrak{c}$.

Demostración. Consideremos el espacio compacto $I^{\mathfrak{c}}$, donde $I=[0,1]$. Dado que $d(I)=\omega$, del teorema de Hewitt-Marczewski-Pondiczery se sigue que $d\left(I^{\mathfrak{c}}\right)=\omega$. Tomemos un conjunto $A \subset I^{\mathfrak{c}}$ tal que $\bar{A}=I^{\mathfrak{c}}$ y $|A|=\omega$. Sea $f: \omega \rightarrow I^{\mathfrak{c}}$ cualquier sobreyección. Como $\omega$ es discreto, $f$ es continua y por lo tanto, existe una función $F: \beta \omega \rightarrow I^{\mathfrak{c}}$ que verifica $F\lceil\omega=f$. Puesto que $\overline{F(\beta \omega)}=F(\beta \omega) \supset F(\omega)=A$, tenemos que $F(\beta \omega)=\overline{F(\beta \omega)} \supset \bar{A}=I^{\mathfrak{c}}$. Así que $F(\beta \omega)=I^{\mathfrak{c}}$, y por lo tanto $|\beta \omega| \geq\left|I^{\mathfrak{c}}\right|=2^{\mathfrak{c}}$. Por otro lado sabemos que $|\beta \omega| \leq 2^{2^{d(\beta \omega)}}=2^{2^{\omega}}=2^{\mathfrak{c}}$. De ambas desigualdades concluimos que $|\beta \omega|=2^{\mathfrak{c}}$. Para la segunda parte del teorema, observemos que de la continuidad de $F$ se desprende que $\mathrm{w}(\beta \omega) \geq \mathrm{w}\left(I^{\mathfrak{c}}\right)=\mathfrak{c}$. Tomando en cuenta que la familia $\{\widehat{U}\}_{U \subset \omega}$ es una base para $\mathcal{T}(\beta \omega)$, obtenemos la desigualdad $\mathrm{w}(\beta \omega) \leq \mathfrak{c}$. Por lo tanto $\mathrm{w}(\beta \omega)=\mathfrak{c}$.

1.41. Lema. Sea $X$ un espacio regular y $F \subset X$ infinito. Luego, existe una familia ajena $\left\{V_{i}\right\}_{i \in \omega} \subset \mathcal{T}(X)$, tal que $\overline{V_{i}} \cap \overline{V_{j}}=\emptyset$ con $i \neq j$ y $V_{i} \cap F \neq \emptyset$ para todo $i \geq 1$.

Demostración. Usaremos inducción. Sea $V_{0}=\emptyset$. Supongamos que tenemos los subconjuntos abiertos $V_{0}, V_{1}, \ldots, V_{n-1}$, tales que la familia $\left\{\overline{V_{0}}, \overline{V_{1}}, \ldots, \overline{V_{n-1}}\right\}$ es ajena, $V_{k} \cap F \neq \emptyset$ con $1 \leq k \leq n-1$ y $F_{n-1}=F-\bigcup_{k=0}^{n-1} \overline{V_{k}}$ es un conjunto infinito. 
Elijamos $a, b \in F_{n-1}$ con $a \neq b$. Por regularidad de $X$ existen $U, V \in \mathcal{T}^{*}(X)$ para los cuales

$$
a \in U \subset \bar{U} \subset X-\left(\bigcup_{k=0}^{n-1} \overline{V_{k}} \cup\{b\}\right) \quad \text { y } \quad b \in V \subset \bar{V} \subset X-\left(\bigcup_{k=0}^{n-1} \overline{V_{k}} \cup \bar{U}\right)
$$

Sea $V_{n}= \begin{cases}U & \text { si } \bar{U} \cap F \text { es finito } \\ V & \text { de otra forma. }\end{cases}$

Luego, la sucesión $\left\{V_{i}\right\}_{i \in \omega}$ así obtenida satisface lo requerido.

1.42. Proposición. Todo conjunto cerrado e infinito $F \subset \beta \omega$ contiene un subconjunto homeomorfo a $\beta \omega$. En particular, todo subconjunto cerrado e infinito de $\beta \omega$ tiene cardinalidad $2^{\mathfrak{c}}$.

Demostración. Consideremos la sucesión $\left\{V_{i}\right\}_{i \in \mathbb{N}}$ proporcionada por el lema previo y el conjunto $A=\left\{a_{i}\right\}_{i \in \mathbb{N}}$ donde $a_{i} \in F \cap V_{i}$ para todo $i \in \mathbb{N}$. Como $A$ es homeomorfo a $\omega$, se tiene que $\beta A$ y $\beta \omega$ son homeomorfos. Debido a que $\bar{A} \subset F$, basta con probar que $\bar{A}$ es homeomorfo a $\beta A$.

Sea $f: A \rightarrow I$ una función continua. Consideremos la función $\widehat{f}: \omega \rightarrow I$ dada por

$$
\widehat{f}(n)= \begin{cases}f\left(a_{i}\right) & \text { si } n \in V_{i} \cap \omega \\ 0 & \text { de otra forma. }\end{cases}
$$

Sea $\widehat{\phi}: \beta \omega \rightarrow I$ la extensión continua de $\widehat{f}$. Afirmamos que la función $\widehat{\phi}$ también extiende a $f$. En efecto, sea $a_{i} \in A$. Para todo $i$ tenemos que $a_{i} \in \overline{V_{i}}=\overline{V_{i} \cap \omega}$, así que

$$
\widehat{\phi}\left(a_{i}\right) \in \widehat{\phi}\left(\overline{V_{i} \cap \omega}\right) \subset \overline{\widehat{\phi}\left(V_{i} \cap \omega\right)}=\overline{\widehat{f}\left(V_{i} \cap \omega\right)}=\left\{f\left(a_{i}\right)\right\},
$$

por lo tanto $\widehat{\phi}\lceil A=f$. Ésto muestra en particular que $f$ se extiende continuamente sobre $\bar{A}$; pues si $\phi=\widehat{\phi}\lceil\bar{A}$ entonces $\phi\lceil A=\widehat{\phi}\lceil A=f$. Por lo tanto la compactificación $\bar{A}$ de $A$ es equivalente a $\beta A$ (corolario 1.9).

1.43. Lema. Para cualesquiera $A, B \subset \omega$ tenemos que $[A] \subset[B] \Longleftrightarrow|A-B|<\omega$.

Demostración. Supongamos que $A-B$ es infinito. Existe un ultrafiltro $\xi$ en $\omega$ tal que $A-B \in \xi$. Luego, $A \in \xi$. Puesto que $(A-B) \cap B=\emptyset$, se tiene que $B \notin \xi$. Por lo tanto $[A] \not \subset[B]$. Para probar la otra implicación, tomemos $\xi \in[A]$. Puesto que el conjunto $C=A-B$ es finito, vemos que $A-C \in \xi$. De aquí resulta que $B \in \xi$, es decir, $\xi \in[B]$. 
1.44. Teorema. Si $G$ es un subespacio no vacío y $G_{\delta}$ de $\omega^{*}$, entonces $G$ tiene interior no vacío en $\omega^{*}$.

Demostración. Sea $G$ un subespacio no vacío y $G_{\delta}$ de $\omega^{*}$. Dado que $\beta \omega$ tiene una base de subconjuntos abiertos-cerrados, se deduce que $\omega^{*}$ tiene una base subconjuntos abiertos y cerrados. De aquí podemos suponer, sin perder la generalidad, que $G=\bigcap_{n \in \mathbb{N}} U_{n}$ con $U_{n+1} \subset U_{n}, U_{n}$ abierto y cerrado en $\omega^{*}$ para todo $n \in \mathbb{N}$.

Para cada $n$ tomemos $U_{n}=\left[A_{n}\right]$ donde $A_{n} \subset \omega$ es infinito. De acuerdo al lema 1.43 podemos afirmar que $\left|A_{n+1}-A_{n}\right|<\omega$. Consideremos la familia $\left\{A_{n}^{\prime}\right\}_{n \in \mathbb{N}}$, donde $A_{n}^{\prime}=\bigcap_{i \leq n} A_{i}$. Dado que $A_{n}^{\prime} \cap A_{n+1}^{\prime} \subset A_{n+1}^{\prime}$ y $\left|A_{n+1}^{\prime}-A_{n}^{\prime}\right|<\omega$, cada conjunto $A_{n}^{\prime}$ es infinito. Sea $A=\left\{x_{n}\right\}_{n \in \mathbb{N}}$ donde $x_{n} \in A_{n}^{\prime}$ y $x_{n} \neq x_{m}$ para $n \neq m$. Si tomamos en cuenta que $\left|A-A_{n}^{\prime}\right|<\omega$, el lema nos garantiza que $[A] \subset\left[A_{n}^{\prime}\right]$ para todo $n \in \mathbb{N}$. Puesto que $\left[A_{n}\right] \subset\left[A_{n}\right]$, tenemos que $[A] \subset\left[A_{n}\right]$ para todo $n \in \mathbb{N}$. Por lo tanto $G \supset[A] \in \mathcal{T}^{*}\left(\omega^{*}\right)$.

Sea $X$ un espacio topológico. El conjunto $A \subset X$ se llama denso en ninguna parte en $X$ si int $\bar{A}=\emptyset$.

1.45. Lema. Supongamos válida la Hipótesis del Continuo. Si $F_{\alpha}$ es un subconjunto cerrado de $\omega^{*}$ e int $F_{\alpha}=\emptyset$ para todo $\alpha<\omega_{1}$ entonces $\omega^{*} \neq \bigcup_{\alpha<\omega_{1}} F_{\alpha}$.

Demostración. Dado que $F_{\alpha}$ es denso en ninguna parte en $\omega^{*}$ para todo $\alpha<\omega_{1}$ y por el teorema 1.27 , el conjunto $G_{\alpha}=\omega^{*}-\bigcup_{\beta \leq \alpha} F_{\beta}=\bigcap_{\beta \leq \alpha}\left(\omega^{*}-F_{\beta}\right)$ es denso en $\omega^{*}$ para todo $\alpha<\omega_{1}$.

Sea $\alpha<\omega_{1}$. Tomemos $U_{0}=G_{0}$ y supongamos que tenemos la familia $\left\{U_{\beta}: \beta<\right.$ $\alpha\} \subset \mathcal{T}\left(\omega^{*}\right)$ tal que $\bar{U}_{\gamma} \subset U_{\beta}$ donde $\beta<\gamma<\alpha$. Luego, el conjunto $\bigcap_{\beta<\alpha} U_{\beta}=$ $\bigcap_{\beta<\alpha} \bar{U}_{\beta}$ es un subconjunto $G_{\delta}$ y no vacío de $\omega^{*}$. El teorema 1.44 garantiza la existencia de abiertos no vacíos $U_{\alpha}^{\prime}$, $U_{\alpha}^{\prime \prime}$ para los cuales $U_{\alpha}^{\prime} \subset \bigcap_{\beta<\alpha} U_{\beta}$ y $U_{\alpha}^{\prime \prime} \subset$ $G_{\alpha} \cap U_{\alpha}^{\prime}$. Tomemos un $U_{\alpha} \in \mathcal{T}^{*}\left(\omega^{*}\right)$ tal que $\overline{U_{\alpha}} \subset U_{\alpha}^{\prime \prime}$ y por lo tanto $\bar{U}_{\alpha} \subset U_{\beta}$ para todo $\beta<\alpha$. Luego, tenemos la familia centrada $\left\{U_{\alpha}: \alpha<\omega_{1}\right\}$ tal que $\bar{U}_{\beta} \subset U_{\alpha}$ para $\alpha<\beta<\omega_{1}$. Como $\bigcap_{\alpha<\omega_{1}} U_{\alpha}=\bigcap_{\alpha<\omega_{1}} \bar{U}_{\alpha} \neq \emptyset$, elijamos un $x \in \bigcap_{\alpha<\omega_{1}} U_{\alpha}$. Puesto que $U_{\alpha} \cap F_{\alpha}=\emptyset$ para todo $\alpha<\omega_{1}$, tenemos que $x \notin F_{\alpha}$ para todo $\alpha<\omega_{1}$. Por lo tanto $x \notin \bigcup_{\alpha<\omega_{1}} F_{\alpha}$, es decir, $\omega^{*} \neq \bigcup_{\alpha<\omega_{1}} F_{\alpha}$.

Un punto $x$ en un espacio topológico es llamado punto $P$ si la intersección de cualquier familia numerable de vecindades de $x$ es una vecindad de $x$.

1.46. Teorema. La Hipótesis del Continuo implica que existen puntos $P$ en $\omega^{*}$.

Demostración. Denotemos por $\mathcal{A C}$ la familia de subconjuntos abiertos y cerrados de $\omega^{*}$. Sabemos por el teorema 1.38 (iv) que $|\mathcal{A C}|=\omega_{1}$. Sea $\mathcal{A C}_{\omega}=\left\{\left\{U_{i}\right\}_{i \in \omega}\right.$ : $\left.U_{i} \in \mathcal{A C}\right\}$. Luego, $\left|\mathcal{A C}_{\omega}\right|=|\mathcal{A C}|^{\omega}=\omega_{1}^{\omega}=\omega_{1}$. Sea $\mathcal{A C}_{\omega}=\left\{\gamma_{\beta}: \beta<\omega_{1}\right\}$. Para cada $\gamma_{\alpha} \in \mathcal{A C}_{\omega}$, tomemos $F_{\alpha}=$ Frontera $\left(\bigcup \gamma_{\alpha}\right)$. Como $F_{\alpha}$ es la frontera de un conjunto 
abierto, tenemos que $F_{\alpha}$ es denso en ninguna parte en $\omega^{*}$ para todo $\alpha<\omega_{1}$. Por el lema 1.45, existe $x \in \omega^{*}-\bigcup_{\alpha<\omega_{1}} F_{\alpha}$. Probemos que $x$ es un punto $P$ en $\omega^{*}$. Sea $U_{n} \in$ $\mathcal{T}\left(x, \omega^{*}\right)$ con $n \in \mathbb{N}$. Podemos suponer sin pérdida de generalidad que $U_{n} \in \mathcal{A C}$ y $U_{n+1} \subset U_{n}$ para todo $n \in \mathbb{N}$. Sea $W_{n}=\omega^{*}-U_{n}$. Luego $\gamma_{\sigma}=\left\{W_{n}\right\}_{n \in \mathbb{N}} \in \mathcal{A C}_{\omega}$ para algún $\sigma<\omega_{1}$. De aquí resulta que $x \notin \bigcup_{n \in \mathbb{N}} W_{n}$ y $x \notin$ Frontera $\left(\bigcup \gamma_{\sigma}\right)$. Lo anterior implica que $x \notin \overline{\bigcup_{n \in \mathbb{N}} W_{n}}$. Por lo tanto, $x \in \omega^{*}-\overline{\bigcup_{n \in \mathbb{N}} W_{n}}=\omega^{*}-$ $\left(\overline{\omega^{*}-\bigcap_{n \in \mathbb{N}} U_{n}}\right)=\operatorname{int}\left(\bigcap_{n \in \mathbb{N}} U_{n}\right)$, de donde $x$ es un punto $P$.

1.47. Teorema. Sean $A$ y $B$ subconjuntos numerables de $\omega^{*}$. Si $A \cap \bar{B}=\bar{A} \cap B=\emptyset$ entonces $\bar{A} \cap \bar{B}=\emptyset$.

Demostración. Sean $A=\left\{a_{n}\right\}_{n \in \mathbb{N}}$ y $B=\left\{b_{n}\right\}_{n \in \mathbb{N}}$. Por ser $\beta \omega$ un espacio normal, para cada $n \in \mathbb{N}$ existen $T_{n}, U_{n}, V_{n}, W_{n} \in \mathcal{T}(\beta \omega)$ tales que $T_{n} \cap U_{n}=$ $V_{n} \cap W_{n}=\emptyset, a_{n} \in T_{n}, \bar{B} \subset U_{n}, \bar{A} \subset V_{n}$ y $b_{n} \in W_{n}$. Tomemos los abiertos $G_{n}=$ $T_{n} \cap\left(\bigcap_{k \leq n} V_{k}\right)$ y $H_{n}=W_{n} \cap\left(\bigcap_{k \leq n} U_{k}\right)$, con $n \in \mathbb{N}$. Así que $a_{n} \in G_{n}, b_{n} \in H_{n}$ y para cualesquier $n, m \in \mathbb{N}$ obtenemos que $G_{n} \cap H_{m}=\emptyset$. Sean $C=\bigcup_{n \in \mathbb{N}} G_{n}$, $D=\bigcup_{n \in \mathbb{N}} H_{n}$. Luego, $C \cap D=\emptyset, A \subset C$ y $B \subset D$. Puesto que $D \cap \bar{C}=\emptyset$ y como $\bar{C}$ es abierto, obtenemos que $\bar{D} \cap \bar{C}=\emptyset$. De aquí concluimos que $\bar{A} \cap \bar{B}=\emptyset$.

Diremos que un subconjunto $A$ de un espacio topológico $X$ es un conjunto cero de $X$ si $A=f^{-1}(0)$ para alguna función continua $f: X \rightarrow[0,1]$. Obviamente todo conjunto cero de $X$ es cerrado en $X$. El complemento de un conjunto cero de $X$ recibe el nombre de conjunto cocero de $X$.

Dado un espacio topológico $X$, consideremos el conjunto $C^{*}(X)=\{f: X \rightarrow$ $\mathbb{R}:$ fes continua y acotada $\}$. Un subconjunto $A \subset X$ se dice que es $C^{*}$ encajado en $X$ si para toda $f \in C^{*}(A)$, existe $F \in C^{*}(X)$ tal que $F\lceil A=f$. Un espacio topológico $X$ es llamado espacio $F$ si todo conjunto cocero $A \subset X$ es $C^{*}$ encajado en $X$.

1.48. Lema. Un subconjunto $A \subset X$ es $C^{*}$ encajado en $X$ si y sólo si cualesquiera subconjuntos $U, V \subset A$ completamente separados en $A$ son completamente separados en $X$.

Demostración. Sean los subconjuntos $U, V \subset A$ completamente separados en $A$. Luego, existe una función continua $f: A \rightarrow[0,1]$ tal que $f(U)=\{0\}$ y $f(V)=\{1\}$. Por hipótesis existe $F: X \rightarrow[0,1]$ para la cual $F\lceil A=f$. Como $F(U)=\{0\}$ y $F(V)=\{1\}$, tenemos que los conjuntos $U$ y $V$ son completamente separados en $X$. Para probar la otra implicación, consideremos una $f \in C^{*}(A)$. Existe $m \in \mathbb{R}$ tal que $|f| \leq m$. Tomemos $f_{1}=f$. Construyamos una sucesión $\left\{f_{n}\right\}_{n \in \mathbb{N}} \subset C^{*}(A)$ tal que $\left|f_{n}\right| \leq 3 r_{n}$ donde $r_{n}=\frac{m}{2}\left(\frac{2}{3}\right)^{n}$. La función $f_{1}$ satisface $\left|f_{1}\right| \leq 3 r_{1}$. Supongamos que tenemos las funciones $f_{i} \in C^{*}(A)$ con $\left|f_{i}\right| \leq 3 r_{i}$ para $i \leq n$. Fijémonos en los conjuntos $A_{n}=\left\{a \in A: f_{n}(a) \leq-r_{n}\right\}$ y $B_{n}=\left\{a \in A: f_{n}(a) \geq r_{n}\right\}$. Luego, $A_{n}$ y $B_{n}$ son completamente separados en $A$. En efecto, la función $F_{n}=\min \{1$, 
$\left.\max \left\{0, \frac{f_{n}}{r_{n}}\right\}\right\}$ verifica $F_{n}\left(A_{n}\right)=\{0\}$ y $F_{n}\left(B_{n}\right)=\{1\}$. Por hipótesis, los conjuntos $A_{n}$ y $B_{n}$ son completamente separados en $X$. Luego, para cada $n \in \mathbb{N}$ existe una función continua $g_{n}: X \rightarrow\left[-r_{n}, r_{n}\right]$ tal que $g_{n}\left(A_{n}\right)=\left\{-r_{n}\right\}$ y $g_{n}\left(B_{n}\right)=\left\{r_{n}\right\}$. Sea $f_{n+1}=f_{n}-g_{n}\left\lceil A\right.$. Tenemos $f_{n+1}(a) \in\left[-2 r_{n}, 0\right]$ cuando $a \in A_{n} ;$ si $a \in B_{n}$ entonces $f_{n+1}(a) \in\left[0,2 r_{n}\right]$; y en el caso $a \notin A_{n} \cup B_{n}$, obtenemos $f_{n+1}(a) \in\left[-2 r_{n}, 2 r_{n}\right]$. De aquí se desprende la desigualdad $\left|f_{n+1}\right| \leq 2 r_{n}$ y como $\frac{r_{n+1}}{r_{n}}=\frac{2}{3}$ concluimos que $\left|f_{n+1}\right| \leq 3 r_{n+1}$. Ésto completa la inducción. Consideremos la función $g$ : $X \rightarrow \mathbb{R}$, dada por $g(x)=\Sigma_{n \in \mathbb{N}} g_{n}(x)$. En virtud de la condición suficiente de Weierstrass, esta serie converge uniformemente. Por lo tanto $g$ es una función continua. Probemos que $g\left\lceil A=f\right.$. Observemos que $\left(g_{1}+g_{2}+\cdots+g_{n}\right)\left\lceil A=\left(f_{1}-\right.\right.$ $\left.f_{2}\right)+\left(f_{2}-f_{3}\right)+\cdots+\left(f_{n}-f_{n+1}\right)=f_{1}-f_{n+1}$ y dado que $f_{n+1}(a) \rightarrow 0$ para todo $a \in A$ concluimos que $g\left\lceil A=f_{1}=f\right.$.

1.49. Teorema. Sea $X$ un espacio normal. Las siguientes condiciones son equivalentes:

(i) Si $U, V \in \mathcal{T}^{*}(X)$ son ajenos y conjuntos $F_{\sigma}$, entonces $\bar{U} \cap \bar{V}=\emptyset$.

(ii) Dada cualquier función continua $f: X \rightarrow \mathbb{R}$, los conjuntos cocero

$A=\operatorname{pos} f=\{x: f(x)>0\}=X-\{x: f(x) \leq 0\}=X-\{x:(f+|f|)(x)=0\} y$

$B=\operatorname{neg} f=\{x: f(x)<0\}=X-\{x: f(x) \geq 0\}=X-\{x:(f-|f|)(x)=0\}$

son completamente separados.

(iii) $X$ es un espacio $F$.

Demostración. Probemos (i) $\Longrightarrow$ (ii). Como los conjuntos abiertos y ajenos $A, B$ son conjuntos cocero en el espacio normal $X$, tenemos que $A$ y $B$ son subconjuntos $F_{\sigma}$ de $X$. Por hipótesis $\bar{A} \cap \bar{B}=\emptyset$. Por ser $X$ un espacio normal, los conjuntos $A$ y $B$ son completamente separados.

Ahora demostremos la implicación (ii) $\Longrightarrow$ (iii).

Sea $A \subset X$ un conjunto cocero de $X$. Consideremos los subconjuntos $U, V \subset A$ completamente separados en $A$. Debido al lema recién probado, basta demostrar que los conjuntos $U$ y $V$ son completamente separados en $X$. Existe $h: X \rightarrow[0,1]$ tal que $A=h^{-1}(0,1]$. Por ser $U, V$ completamente separados en $A$, existe una función acotada $k: A \rightarrow \mathbb{R}$ para la cual $k(U)>0$ y $k(V)<0$. Consideremos la función $f: X \rightarrow \mathbb{R}$ dada por $f(x)=k(x) h(x)$ si $x \in A$; en caso contrario, hagamos $f(x)=0$. Como $k$ es acotada, tenemos que $f$ es una función continua. Claramente se cumple que $U \subset$ pos $f$ y $V \subset$ neg $f$. De la hipótesis se desprende que $U$ y $V$ son completamente separados en $X$. Por el lema, el conjunto $A \subset X$ es $C^{*}$ encajado en $X$.

Ahora probemos la implicación (iii) $\Longrightarrow($ i). Sean $U, V$ subconjuntos abiertos, no vacíos y $F_{\sigma}$ de $X$. Como $X$ es un espacio normal, tenemos que $U$ y $V$ son conjuntos cocero de $X$. Consideremos la función continua $f: U \cup V \rightarrow[0,1]$, dada por 
$f(U)=\{0\}$ y $f(V)=\{1\}$. Por ser $X$ un espacio $F$ y $U \cup V$ un conjunto cocero de $X$, existe una función continua $g: X \rightarrow[0,1]$ tal que $g \mid U \cup V=f$. Luego $U \subset g^{-1}(0)$ y $V \subset g^{-1}(1)$. De aquí concluimos que $\bar{U} \cap \bar{V}=\emptyset$.

1.50. Proposición. Sean $X$ un espacio normal y $A \subset X$ un subespacio cerrado. Si $X$ es un espacio $F$ entonces $A$ es un espacio $F$.

Demostración. Consideremos una función continua $f: A \rightarrow \mathbb{R}$. Probemos que los conjuntos pos $f$ y neg $f$ son separados en $X$. Debido a que $X$ es un espacio normal, existe una función continua $g: X \rightarrow \mathbb{R}$ tal que $g\lceil A=f$. Luego pos $f \subset$ $\operatorname{pos} g$ y neg $f \subset \operatorname{neg} g$. Dado que $X$ es un espacio $F$, existe una función continua $h: X \rightarrow[0,1]$ para la cual $h(\operatorname{pos} g)=\{0\}$ y $h(\operatorname{neg} g)=\{1\}$. La función $H=h\lceil A$ satisface $H(\operatorname{pos} f)=\{0\}$ y $H(\operatorname{neg} f)=\{1\}$. Por lo tanto $A$ es un espacio $F$.

La siguiente proposición es una consecuencia inmediata de 1.38(vi) y 1.49(i)

1.51. Proposición. $\beta \omega$ es un espacio $F$.

De las dos últimas proposiciones obtenemos el siguiente resultado

1.52. Corolario. $\omega^{*}$ es un espacio $F$.

\section{Ultracompletitud}

El siguiente teorema es la base de la definición de los espacios ultracompletos. Sea $X$ un espacio topológico de Tychonoff. Consideremos un subconjunto $A \subset$ $X$. Recordemos que el carácter de $A$ en $X$ está dado por $\chi(A, X)=\min \{|\mathcal{B}|$ : $\mathcal{B}$ es una base para $A$ en $X\}$ (ver pag. 16).

1.52. Teorema. Sea $X$ un espacio de Tychonoff. Las siguientes condiciones son equivalentes:

(a) $\chi(X, c X) \leq \omega$ para toda compactificación $c X$ de $X$;

(b) $\chi(X, \beta X) \leq \omega$ donde $\beta X$ es la compactificación de Stone-Čech de $X$;

(c) $\chi(X, c X) \leq \omega$ para alguna compactificación $c X$ de $X$.

Demostración. Las implicaciones $(\mathrm{a}) \Longrightarrow(\mathrm{b}) \Longrightarrow(\mathrm{c})$ son evidentes.

Probemos $(\mathrm{c}) \Longrightarrow(\mathrm{b})$. En virtud de los Teoremas 1.4 y 1.12, existe una función perfecta $f: \beta X \rightarrow c X$ tal que $f \circ \beta=c$ y $f^{-1}(c X-c(X))=\beta X-c(X)$. Sea $\mathcal{B}=$ $\left\{U_{n}\right\}_{n \in \mathbb{N}}$ una base para $c(X)$ en $c X$. Tomemos la familia $\mathcal{B}^{\prime}=\left\{f^{-1}\left(U_{n}\right)\right\}_{n \in \mathbb{N}} \subset$ $\mathcal{T}(\beta X)$. Mostremos que $\mathcal{B}^{\prime}$ es base para $\beta(X)$ en $\beta X$. Es claro que $\beta(X) \subset f^{-1}\left(U_{n}\right)$ para todo $n \in \mathbb{N}$. Tomemos un $V \in \mathcal{T}(\beta X)$ para el cual $\beta(X) \subset V$. Consideremos el conjunto cerrado $F=\beta X-V$. Luego, el cerrado $f(F)$ satisface $c(X) \subset c X-f(F)$. Existe $U_{n_{0}} \in \mathcal{B}$ tal que $c(X) \subset U_{n_{0}} \subset c X-f(F)$. De aquí se desprende que $\beta(X) \subset f^{-1}\left(U_{n_{0}}\right) \subset f^{-1}(c X-f(F)) \subset \beta X-F=V$. Ahora verifiquemos (b) $\Longrightarrow$ (a). 
Consideremos $\mathcal{B}=\left\{U_{n}\right\}_{n \in \mathbb{N}} \subset \mathcal{T}(\beta X)$ una base para $\beta(X)$ en $\beta X$ y tomemos cualquier compactificación $c X$ de $X$. Existe una función perfecta $f: \beta X \rightarrow c X$ tal que $f \circ \beta=c$ y $f(\beta X-\beta(X))=c X-c(X)$. Sea $n \in \mathbb{N}$. Consideremos $F_{n}=\beta X-U_{n}$. Como la función $f$ es cerrada, tenemos que $V_{n}=c X-f\left(F_{n}\right) \in \mathcal{T}(c X)$. La familia $\left\{V_{n}\right\}_{n \in \mathbb{N}}$ es base para $c(X)$ en $c X$. Es claro que $c(X) \subset V_{n}$ para todo $n \in \mathbb{N}$. Tomemos un $V \in \mathcal{T}(c X)$ para el cual $c(X) \subset V$ De aquí $\beta(X) \subset f^{-1}(V) \in \mathcal{T}(\beta X)$. Existe $U_{n_{0}} \in \mathcal{B}$ tal que $\beta(X) \subset U_{n_{0}} \subset f^{-1}(V)$, de donde $f\left(\beta X-f^{-1}(V)\right) \subset$ $f\left(\beta X-U_{n_{0}}\right)$. Por lo tanto

$$
V_{n_{0}}=c X-f\left(F_{n_{0}}\right)=c X-f\left(\beta X-U_{n_{0}}\right) \subset c X-f\left(\beta X-f^{-1}(V)\right) \subset V .
$$

Un espacio $X$ de Tychonoff es llamado ultracompleto si $X$ satisface cualquiera de las condiciones en el teorema 1.52 ( ver [BY1], [BY2] y [PT]). Es claro de la definición que son válidas las implicaciones: localmente compacto $\Longrightarrow$ ultracompleto $\Longrightarrow$ Čech completo. En [BY1] se proporcionan los siguientes ejemplos que muestran que las implicaciones inversas no son ciertas. La demostración del primero de ellos, también puede obtenerse como consecuencia inmediata de 3.5.

1.53. Ejemplo. Sea $I=[0,1]$ con la topología heredada de $\mathbb{R}$. Consideremos el subespacio $X=I-\left\{\frac{1}{n}: n \geq 2\right\} \subset I$. El espacio $X$ es ultracompleto y no es localmente compacto.

Demostración. Dado que $X$ no es un subconjunto abierto de $I$, se desprende que $X$ no es localmente compacto. Para cada $k \in \mathbb{N}$, sea $U_{k}=I-\left\{\frac{1}{n}: n=2,3, \ldots, k+1\right\}$. Verifiquemos que $\left\{U_{k}\right\}_{k \in \mathbb{N}}$ es base para $X$ en $I$. Claramente, el conjunto $U_{k}$ es abierto en $I$ y $X \subset U_{k}$ para todo $k \in \mathbb{N}$. Sea $X \subset U$ donde $U$ es abierto en $I$. Dado que $0 \in X$, existe $k_{0} \geq 2$ tal que $\left[0, \frac{1}{k_{0}+1}\right] \subset U$. Por lo tanto, obtenemos que $X \subset U_{k_{0}} \subset U$.

Los números irracionales con la topología heredada de $\mathbb{R}$ es un espacio Čech completo (ver teorema 1.13). Del siguiente resultado, y también como consecuencia de 3.5, se desprende que este espacio no es ultracompleto.

1.54. Lema. Sea $A \subset \mathbb{R}$ tal que $\bar{A}=\overline{\mathbb{R}-A}=\mathbb{R}$. Entonces $A$ no es ultracompleto.

Demostración. Supongamos que $A$ es ultracompleto. Probemos primero que $\chi(A, \mathbb{R}) \leq \omega$. Para ello, consideremos la compactificación de Alexandroff $\alpha \mathbb{R}$ de $\mathbb{R}$. Como $A$ es denso en $\mathbb{R}$, tenemos que $\alpha \mathbb{R}$ también es una compactificación de $A$. Por lo tanto $\chi(A, \alpha \mathbb{R}) \leq \omega$. De aquí resulta que $\chi(A, \mathbb{R}) \leq \omega$.

Ahora consideremos una base $\left\{U_{n}\right\}_{n \in \mathbb{N}}$ para $A$ en $\mathbb{R}$. Puesto que $\overline{U_{n}}=\mathbb{R}$, tenemos que $V_{n}=U_{n} \cap\left(n, n+\frac{1}{3}\right) \neq \emptyset$ para todo $n \in \mathbb{N}$. Dado que también se satisface $\overline{\mathbb{R}-A}=\mathbb{R}$, existe algún $a_{n} \in \mathbb{R}-A$ tal que $a_{n} \in V_{n}$ para todo $n \in \mathbb{N}$. Tomemos el subespacio cerrado $D=\left\{a_{n}: n \in \mathbb{N}\right\} \subset \mathbb{R}-A$. Luego, $\mathbb{R}-D$ es un abierto que 
contiene a $A$ y no contiene a $U_{n}$ para todo $n \in \mathbb{N}$, pues $V_{n} \not \subset$ y $V_{n} \subset U_{n}$ con $n \in \mathbb{N}$, lo cual es una contradicción.

La definición de la ultracompletitud del espacio $X$ es una definición que depende de otros espacios: sus compactificaciones. El siguiente teorema proporciona caracterizaciones internas de un espacio ultracompleto.

Dadas dos cubiertas $\mathcal{U}, \mathcal{V} \subset \exp (X)$ de un espacio $X$, decimos que $\mathcal{U}$ es un refinamiento de $\mathcal{V}$, denotándolo por $\mathcal{U} \prec \mathcal{V}$, si para todo $U \in \mathcal{U}$ existe $V \in \mathcal{V}$ tal que $U \subset V$. Para una cubierta abierta $\mathcal{V}$ de $X$ escribiremos $\mathcal{V}^{f}=\{\bigcup \mathcal{W}: \mathcal{W} \subset$ $\mathcal{V}$ y $|\mathcal{W}|<\omega\}$. Se dice que una familia $\mathcal{F} \subset \exp (X)$ se enreda con una sucesión de cubiertas abiertas $\mathcal{U}=\left\{\mathcal{U}_{n}\right\}_{n \in \mathbb{N}}$, si existe una sucesión $\left\{U_{n}\right\}_{n \in \mathbb{N}}$ donde $U_{n} \in \mathcal{U}_{n}$, tal que para todo $F \in \mathcal{F}$ y todo $n \in \mathbb{N}$ se cumple que $F \cap U_{n} \neq \emptyset$.

1.55. Teorema. Sea $X$ un espacio de Tychonoff. Las siguientes condiciones son equivalentes:

(i) $X$ es ultracompleto;

(ii) Existe una sucesión $\mathcal{U}=\left\{\mathcal{U}_{n}\right\}_{n \in \omega}$ de cubiertas abiertas de $X$ tales que si $\mathcal{F}$ es base de filtro que se enreda con $\mathcal{U}$ entonces $\bigcap \overline{\mathcal{F}} \neq \emptyset$;

(iii) Existe una sucesión $\left\{\mathcal{U}_{n}\right\}_{n \in \omega}$ de cubiertas abiertas de $X$ tales que para toda cubierta abierta $\mathcal{V}$ de $X$ existe $n \in \omega$ para el cual $\mathcal{U}_{n} \prec \mathcal{V}^{f}$.

Demostración. (i) $\Longrightarrow$ (ii). Tomemos una compactificación $c X$ de $X$ y una base $\mathcal{B}=\left\{U_{n}\right\}_{n \in \omega} \subset \mathcal{T}(c X)$ para $X$ en $c X$. Sean $x \in X$ y $V_{n}(x) \in \mathcal{T}(x, c X)$ tales que

$$
V_{n}(x) \subset{\overline{V_{n}(x)}}^{c X} \subset U_{n} .
$$

Luego, la familia $\mathcal{V}_{n}=\left\{X \cap V_{n}(x): x \in X\right\}$ es una cubierta abierta de $X$ para todo $n \in \omega$. Afirmamos que la sucesión $\mathcal{V}=\left\{\mathcal{V}_{n}\right\}_{n \in \omega}$ satisface (ii). Sea $\mathcal{F}$ una base de filtro en $X$ que se enreda con $\mathcal{V}$. Tenemos que demostrar que $\cap \overline{\mathcal{F}} \neq \emptyset$.

Notemos que $\overline{\mathcal{F}}^{c X}=\left\{\bar{F}^{c X}: F \in \mathcal{F}\right\}$ es base de filtro en $c X$. Por ser $c X$ compacto tenemos que $\bigcap^{\overline{\mathcal{F}}^{c X}} \neq \emptyset$. Sea $H=\bigcap^{\overline{\mathcal{F}}^{c X}}$. Basta demostrar que $H \cap X \neq \emptyset$. Supongamos lo contrario. Existe $U_{n} \in \mathcal{B}$, para el cual $X \subset U_{n} \subset c X-H$. Dado que existen puntos $x_{k} \in X, k \in \omega$ tales que $F \cap\left(X \cap V_{k}\left(x_{k}\right)\right) \neq \emptyset$ para todo $F \in \mathcal{F}$ y todo $k \in \omega$, se obtiene

$$
\emptyset \neq{\overline{F \cap\left(X \cap V_{n}\left(x_{n}\right)\right)}}^{c X} \subset \bar{F}^{c X} \cap{\overline{\left(X \cap V_{n}\left(x_{n}\right)\right)}}^{c X}=\bar{F}^{c X} \cap{\overline{V_{n}\left(x_{n}\right)}}^{c X}
$$

Como $\left\{\bar{F}^{c X} \cap{\overline{V_{n}\left(x_{n}\right)}}^{c X}: F \in \mathcal{F}\right\}$ es base de filtro en $c X$ se tiene

$$
\bigcap_{F \in \mathcal{F}}\left(\bar{F}^{c X} \cap{\overline{V_{n}\left(x_{n}\right)}}^{c X}\right) \neq \emptyset .
$$


Lo anterior implica que $H \cap U_{n} \neq \emptyset$, lo que es una contradicción.

(ii) $\Longrightarrow$ (iii) Sea $\left\{\mathcal{U}_{n}\right\}_{n \in \omega}$ una sucesión de cubiertas abiertas de $X$ que constata (ii). Demostremos que esta sucesión satisface (iii). Consideremos una cubierta abierta $\mathcal{V}$ de $X$. Si $\mathcal{V}$ contiene una subcubierta finita de $X$ entonces $\mathcal{U}_{n} \prec \mathcal{V}^{f}$ para todo $n \in \omega$. Luego, supongamos que para toda $\mathcal{V}^{\prime} \in \mathcal{V}^{f}$ se cumple que $X-\mathcal{V}^{\prime} \neq \emptyset$. De lo anterior se sigue que la familia de subconjuntos cerrados $\mathcal{F}=\left\{X-\mathcal{V}^{\prime}: \mathcal{V}^{\prime} \in \mathcal{V}^{f}\right\}$ es base de filtro en $X$.

Supóngase que no existe $n \in \omega$ tal que $\mathcal{U}_{n} \prec \mathcal{V}^{f}$. Por lo tanto, para toda $n \in \omega$ existe $U \in \mathcal{U}_{n}$ tal que $U \cap\left(X-\mathcal{V}^{\prime}\right) \neq \emptyset$ para todo $\mathcal{V}^{\prime} \in \mathcal{V}^{f}$. Así que, de la hipótesis obtenemos que

$$
\emptyset \neq \bigcap \mathcal{F}=\bigcap_{\mathcal{V}^{\prime} \in \mathcal{V}^{f}}\left(X-\mathcal{V}^{\prime}\right)=X-\bigcup_{\mathcal{V}^{\prime} \in \mathcal{V}^{f}} \mathcal{V}^{\prime}
$$

Lo anterior contradice que $X=\bigcup \mathcal{V}^{f}$.

(iii) $\Longrightarrow$ (i). Sea $c X$ una compactificación de $X$. Tomemos $\left\{\mathcal{U}_{n}\right\}_{n \in \omega}$ una sucesión que garantiza la validez de (iii). Observemos que para para todo $U \in \mathcal{U}_{n} \operatorname{con} n \in \omega$, se cumple que $U \subset \operatorname{int}_{c X} \bar{U}^{c X}$. En efecto, sea $V \in \mathcal{T}(c X)$ para el cual $U=X \cap V$. Luego $\bar{U}^{c X}=\bar{V}^{c X}$. Por lo tanto $U \subset V \subset \bar{U}^{c X}$, concluyéndose de esta forma que $U \operatorname{cint}_{c X} \bar{U}^{c X}$.

Para cada $n \in \mathbb{N}$ hagamos $U_{n}=\bigcup_{U \in \mathcal{U}_{n}}\left(\right.$ int $\left._{c X} \bar{U}^{c X}\right)$. Es claro que $U_{n} \in \mathcal{T}(c X)$ para toda $n$ y puesto que $X \subset \bigcup_{U \in \mathcal{U}_{n}} U$ obtenemos $X \subset \bigcup_{U \in \mathcal{U}_{n}} U_{n}$. Afirmamos que la familia $\left\{U_{n}\right\}_{n \in \mathbb{N}}$ es base para $X$. En efecto, Sea $V \in \mathcal{T}(c X)$ para el cual $X \subset V$.

Para cada $x \in X$ existen abiertos ajenos $W_{x}$ y $V_{x}$ tales que $x \in W_{x}$ y $c X-V \subset V_{x}$. Tenemos que $\mathcal{W}=\left\{X \cap W_{x}: x \in X\right\}$ es una cubierta abierta de $X$. Por hipótesis existe $n \in \omega$ para el cual $\mathcal{U}_{n} \prec \mathcal{W}^{f}$. De aquí se sigue que para todo $U \in \mathcal{U}_{n}$ existen $n(U) \in \omega, W_{i}(U) \in \mathcal{W}$ y abiertos $V_{i}(U)$ para $i \leq n(U)$ tales que

$$
\begin{gathered}
U \subset \bigcup_{i=0}^{n(U)} W_{i}(U) \quad c X-V \subset \bigcap_{i=0}^{n(U)} V_{i}(U) \mathrm{y} \\
\left(\bigcup_{i=0}^{n(U)} W_{i}(U)\right) \bigcap\left(\bigcap_{i=0}^{n(U)} V_{i}(U)\right)=\emptyset
\end{gathered}
$$


De aquí $\bar{U}^{c X} \subset V$ para todo $U \in \mathcal{U}_{n}$. Por lo anterior y como $U \subset$ int ${ }_{c X} \bar{U}^{c X} \subset \bar{U}^{c X}$ obtenemos

$$
U_{n}=\bigcup_{U \in \mathcal{U}_{n}} \widehat{U} \subset \bigcup_{U \in \mathcal{U}_{n}} \bar{U}^{c X} \subset V
$$

Diremos que la sucesión $\mathcal{U}=\left\{\mathcal{U}_{n}\right\}_{n \in \mathbb{N}}$ de cubiertas abiertas de $X$ es ultracompleta si satisface la condición (ii) del teorema 1.55. Por lo tanto, un espacio $X$ es ultracompleto si posee una sucesión ultracompleta de cubiertas abiertas.

1.56. Proposición. La ultracompletitud se hereda a subconjuntos cerrados.

Demostración. Sea $X$ un espacio ultracompleto. Consideremos un subespacio cerrado $F \subset X$ y una sucesión $\mathcal{U}=\left\{\mathcal{U}_{n}\right\}_{n \in \mathbb{N}}$ de cubiertas abiertas de $X$ que satisface la propiedad (iii) del teorema 1.55. Sea $\mathcal{U}_{F}=\left\{\mathcal{U}_{n}^{F}\right\}_{n \in \mathbb{N}}$, donde $\mathcal{U}_{n}^{F}=$ $\left\{F \cap U: U \in \mathcal{U}_{n}\right\}$. Verifiquemos que la sucesión de cubiertas abiertas $\mathcal{U}_{F}$ de $F$ satisface la propiedad mencionada. Tomemos una cubierta abierta $\mathcal{V}$ de $F$ y consideremos la cubierta abierta $\mathcal{W}=\{U \in \mathcal{T}(X): F \cap U \in \mathcal{V}\} \cup\{X-F\}$ de $X$. Por hipótesis, existe un $n_{0} \in \mathbb{N}$ tal que $\mathcal{U}_{n_{0}} \prec \mathcal{W}^{f}$. Luego, para cualquier $U \in \mathcal{U}_{n_{0}}$ con $F \cap U \neq \emptyset$, existen $m \in \mathbb{N}$ y $W_{i} \in \mathcal{W}, i \leq m$, tales que $U \subset \bigcup_{i \leq m} W_{i}$. De lo anterior resulta que $\mathcal{U}_{n_{0}}^{F} \ni F \cap U \subset\left(\bigcup_{i \leq m} F \cap W_{i}\right) \in \mathcal{V}^{f}$ y por lo tanto $\mathcal{U}_{n_{0}}^{F} \prec \mathcal{V}^{f}$.

1.57. Teorema. Sea $f: X \rightarrow Y$ una función abierta y sobre. Si el espacio $X$ es ultracompleto entonces $Y$ es ultracompleto.

Demostración. Sea $\mathcal{U}=\left\{\mathcal{U}_{n}\right\}_{n \in \mathbb{N}}$ una sucesión ultracompleta de cubiertas abiertas de $X$. Tomemos $\mathcal{W}=\left\{\mathcal{W}_{n}\right\}_{n \in \mathbb{N}}$ donde $\mathcal{W}_{n}=\left\{W \in \mathcal{T}^{*}(Y): W \subset f\left(U^{n}\right)\right.$ con $\left.U^{n} \in \mathcal{U}_{n}\right\}$. Es claro que $\mathcal{W}_{n} \subset \mathcal{T}(Y)$ y $Y=\bigcup \mathcal{W}_{n}$ para todo $n \in \mathbb{N}$. Verifiquemos que la sucesión $\mathcal{W}$ es ultracompleta. Sea $\mathcal{F}$ una base de filtro que se enreda $\operatorname{con} \mathcal{W}$. Podemos suponer, sin pérdida de generalidad que todos los elementos de $\mathcal{F}$ son subconjuntos cerrados de $Y$. Existe una sucesión $\left\{W_{n}\right\}_{n \in \mathbb{N}}$ con $W_{n} \in \mathcal{W}_{n}$ para la cual $F \cap W_{n} \neq \emptyset$ para todo $F \in \mathcal{F}$ y $n \in \mathbb{N}$. Luego, la base de filtro $f^{-1}(\mathcal{F})$ se enreda con $\mathcal{U}$. Por hipótesis, existe $x \in \bigcap_{F \in \mathcal{F}} f^{-1}(F) \neq \emptyset$. Por lo tanto, $f(x) \in \bigcap_{F \in \mathcal{F}} F$.

1.58. Teorema. Sea $f: X \rightarrow Y$ una función perfecta y sobre. El espacio $X$ es ultracompleto si y sólo si $Y$ es ultracompleto.

Demostración. Supongamos que el espacio $Y$ es ultracompleto. Existe una base numerable $\left\{V_{n}\right\}_{n \in \mathbb{N}}$ de $Y$ en $\beta Y$. Del teorema 1.23, la extensión continua $F: \beta X \rightarrow$ $\beta Y$ de $f$ satisface $F(\beta X-X) \subset \beta Y-Y$. Luego, obtenemos que $X \subset F^{-1}\left(V_{n}\right)$ para todo $n \in \mathbb{N}$. Sea $G \in \mathcal{T}(\beta X)$ tal que $X \subset G$. Como $F(\beta X-G) \subset \beta Y-Y$, tenemos que $Y \subset \beta Y-F(\beta X-G)$. Existe $m \in \mathbb{N}$ para el cual $Y \subset V_{m} \subset Y-F(\beta X-G)$. De aquí se desprende que $X \subset F^{-1}\left(V_{m}\right) \subset G$. Por lo tanto $\left\{F^{-1}\left(V_{n}\right)\right\}_{n \in \mathbb{N}}$ es 
base para $X$ en $\beta X$. Ahora bien, si $X$ es ultracompleto entonces existe una base numerable $\left\{U_{n}\right\}_{n \in \mathbb{N}}$ de $X$ en $\beta X$. Para cada $n \in \mathbb{N}$, sea $K_{n}=\beta X-U_{n}$. Luego, $Y \subset \beta Y-F\left(K_{n}\right)=W_{n}$, donde $F$ es la función mencionada con anterioridad. Cada conjunto $W_{n}$ es abierto y contiene a $Y$. Sea $V$ un abierto en $\beta Y$ que contiene a $Y$. Luego $X \subset \beta X-F^{-1}(\beta Y-V)$. Existe $m \in \mathbb{N}$ para el cual $X \subset U_{m} \subset$ $\beta X-F^{-1}(\beta Y-V)$. Por lo tanto $F^{-1}(\beta Y-V) \subset K_{m} \subset \beta X-X$. Esto implica que $Y \subset W_{m} \subset V$. Es decir, la familia $\left\{W_{n}\right\}_{n \in \mathbb{N}}$ es base para $Y$ en $\beta Y$. 


\section{Capítulo 2}

\section{Aditividad y algunas otras propiedades de la Čech completitud}

En este capítulo, proporcionamos algunos resultados que hemos obtenido con respecto a la aditividad de la Čech completitud. La Čech completitud no es una propiedad aditiva. En el ejemplo 2.7 mostramos un espacio topológico $X$ que es la unión de dos subespacios Čech completos sin ser Čech completo. Probamos que si un espacio $X$ posee una cubierta abierta localmente finita de subespacios Cech completos entonces $X$ es Čech completo (teorema 2.8). En el caso de los grupos topológicos, demostramos que si un grupo topológico es unión finita de subespacios Čech completos entonces es Čech completo (teorema 2.29). Se dice que una propiedad $\mathcal{P}$ es $n$-aditiva (en la potencia $n \in \mathrm{N}$ ) si $X \in \mathcal{P}$ siempre que $X^{n}=X_{1} \cup X_{2} \cup \ldots \cup X_{n}$, donde $X_{i} \in \mathcal{P}$ cuando $i \leq n$. La propiedad $\mathcal{P}$ es aditiva en potencias finitas si es $n$-aditiva para todo $n \in \mathbb{N}$. Establecemos que la propiedad de tipo puntual numerable es aditiva en potencias finitas (corolario 2.5) y proporcionamos un ejemplo que muestra que es consistente con ZFC que la Cech completitud no es aditiva en potencias finitas (ejemplo 2.26). Existen muchos resultados referentes a la aditividad en potencias numerables. Tkachuk demostró en $[\mathrm{TkV}]$ que la metrizabilidad es finitamente aditiva en potencias numerables. En [OY] se prueba que la paracompacidad también cumple con esta propiedad. En el presente capítulo demostramos que la completitud según Čech también es finitamente aditiva en potencias numerables (teorema 2.20).

\section{Aditividad finita de la Čech completitud.}

En esta sección estableceremos algunos resultados acerca de la aditividad finita de la Čech completitud en potencias finitas. Probaremos que la Čech completitud es aditiva en espacios metrizables. También demostramos que la Čech completitud es finitamente aditiva en $X^{\omega}$. El primer resultado que proporcionamos se refiere a la aditividad en potencias finitas de la propiedad de tipo puntual numerable. Para ello, necesitaremos de los siguientes resultados (véase [Ar2]). 
2.1. Teorema. Sea $X$ un espacio topológico.

(i) Si $X$ es de tipo puntual numerable entonces $X$ es la unión de subconjuntos $G_{\delta}$ en cualquier compactificación $c X$.

(ii) Sea $K$ un espacio compacto que contiene a $X$. Si $X$ es la unión de subconjuntos $G_{\delta}$ en $K$ entonces $X$ es de tipo puntual numerable.

Demostración. Probemos (i). Como $X$ es de tipo puntual numerable, se sigue que $X=\cup_{x \in X} K_{x}$, donde $x \in K_{x}$ y $K_{x}$ es compacto tal que $\chi\left(K_{x}, X\right) \leq \omega$. Sea $c X$ una compactificación de $X$. Dado que $K_{x}$ es un conjunto $G_{\delta}$ de $X$ también lo es en $c X$. En efecto, verifiquemos que $\chi\left(K_{x}, c X\right) \leq \omega$. Sea $K_{x}=\bigcap_{i \in \mathbb{N}} U_{i}$ con $U_{i} \in \mathcal{T}(X), i \in \mathbb{N}$. Consideremos los conjuntos $V_{i} \in \mathcal{T}(c X)$ tal que $U_{i}=X \cap V_{i}$ para todo $i \in \mathbb{N}$. Ahora bien, verifiquemos que la familia $\mathcal{V}=\left\{V_{i}\right\}_{i \in \mathbb{N}} \subset \mathcal{T}(c X)$ es base para $K_{x}$. Sea $V \in \mathcal{T}\left(K_{x}, c X\right)$. Por ser $K_{x}$ compacto, existe $U \in \mathcal{T}(c X)$ para el cual $K_{x} \subset U \subset \bar{U} \subset V$. Luego $K_{x} \subset X \cap U$ y por lo tanto, existe $V_{i_{0}} \in \mathcal{V}$ para algún $i_{0} \in \mathbb{N}$ tal que $K_{x} \subset X \cap V_{i_{0}} \subset X \cap U$. De aquí resulta que

$$
K_{x} \subset V_{i_{0}} \subset \overline{V_{i_{0}}}=\overline{X \cap V_{i_{0}}} \subset \overline{X \cap U}=\bar{U} \subset V .
$$

Con lo que $\mathcal{V}$ es base para $K_{x}$ y por lo tanto $\chi\left(K_{x}, c X\right) \leq \omega$.

Ahora probemos (ii). Sea $K$ un compacto que contiene a $X$ y supongamos que $X=\cup_{\alpha \in \mathcal{I}} M_{\alpha}$ donde para cada $\alpha \in \mathcal{I}$ se tiene que $M_{\alpha}=\bigcap_{i \in \mathbb{N}} U_{i}^{\alpha} \operatorname{con} U_{i}^{\alpha} \in \mathcal{T}(K)$ e $i \in \mathbb{N}$. Fijemos un $x \in X$. Luego $x \in M_{\alpha_{x}}$ para algún $\alpha_{x} \in \mathcal{I}$. Por regularidad, podemos obtener la sucesión $\left\{V_{i}^{\alpha_{x}}\right\}_{i \in \mathbb{N}} \subset \mathcal{T}(K)$ que satisface las propiedades

(a) $x \in V_{i}^{\alpha_{x}} \subset U_{i}^{\alpha_{x}}$ para todo $i \in \mathbb{N}$ y

(b) $\overline{V_{j}^{\alpha_{x}}} \subset V_{i}^{\alpha_{x}}$ para $j>i$.

Consideremos el conjunto compacto $F=\bigcap_{i \in \mathbb{N}} V_{i}^{\alpha_{x}}=\bigcap_{i \in \mathbb{N}} \overline{V_{i}^{\alpha_{x}}}$. Nótese que $x \in F$. De la propiedad (a) se desprende que $F \subset X$ y como $F$ es un conjunto $G_{\delta}$ de $K$, también lo es de $X$. Concluyendo de esta forma que $\chi(F, X) \leq \omega$.

2.2. Coroloario. La propiedad de tipo puntual numerable se hereda a subconjuntos cerrados y a subconjuntos $G_{\delta}$.

Demostración. Sean $X$ un espacio de tipo puntual numerable y $c X$ una compactificación de $X$. Consideremos un subconjunto cerrado $F \subset X$. Por el teorema 2.1 (i), tenemos que $X=\bigcup_{\alpha \in \mathcal{I}} H_{\alpha}$ donde $H_{\alpha}$ es un subconjunto $G_{\delta}$ de $c X$. Para cada $\alpha \in \mathcal{I}$, tomemos $F_{\alpha}=F \cap H_{\alpha}$. Como $F$ es cerrado, tenemos que $F_{\alpha}=\bar{F}^{c X} \cap H_{\alpha}, \mathrm{y}$ por lo tanto $F_{\alpha}$ es un subconjunto $G_{\delta}$ de $\bar{F}^{c X}$. Dado que $F=\bigcup_{\alpha \in \mathcal{I}} F_{\alpha}$, del teorema 2.1 (ii) se desprende que $F$ es de tipo puntual numerable. Ahora, sea $G \subset X$ un subconjunto $G_{\delta}$ de $X$. Consideremos $G=\bigcap_{i \in \mathbb{N}} U_{i}$ donde $U_{i} \in \mathcal{T}(X)$. Para cada $i \in \mathbb{N}$, escojamos $V_{i} \in \mathcal{T}(c X)$ tal que $V_{i}=X \cap U_{i}$. Dado que para cada $\alpha \in \mathcal{I}$, el conjunto $H_{\alpha}$ es $G_{\delta}$ en $c X$, tenemos que $W_{\alpha}=H_{\alpha} \cap G=H_{\alpha} \cap\left(\bigcap_{i \in \mathbb{N}} X \cap V_{i}\right)=$ 
$H_{\alpha} \bigcap\left(\bigcap_{i \in \mathbb{N}} V_{i}\right)$ es un subconjunto $G_{\delta}$ de $c X$. De la igualdad $G=\bigcup_{\alpha \in \mathcal{I}} W_{\alpha}$ y del teorema 2.1 (ii), se sigue que $G$ es de tipo puntual numerable.

2.3. Lema. Si $X=A_{1} \cup A_{2} \cup \ldots \cup A_{n}$ donde $A_{i}$ es de tipo puntual numerable para toda $i \in\{1,2, \ldots, n\}$, entonces $\bigcap_{i=1}^{n} \overline{A_{i}}$ es de tipo puntual numerable.

Demostración. Por el teorema 2.1 (ii), es suficiente probar que $\bigcap_{i=1}^{n} \overline{A_{i}}$ es unión de subconjuntos $G_{\delta}$ del compacto $P=\bigcap_{i=1}^{n}{\overline{A_{i}}}^{\beta X}$.

Como $A_{i}$ es de tipo puntual numerable para toda $i \in \mathbb{N}$, se cumple que $A_{i}=$ $\bigcup_{\alpha \in \mathcal{I}_{i}} G_{\alpha}^{i}$ donde $G_{\alpha}^{i}$ es un subespacio $G_{\delta}$ de ${\overline{A_{i}}}^{\beta X}$ para toda $\alpha \in \mathcal{I}_{i}$ e $i=1,2, \ldots, n$. Consideremos $A_{i}^{\prime}=A_{i} \cap P$ para cada $i \leq n$. Luego $A_{i}^{\prime}=\bigcup_{\alpha \in \mathcal{I}_{i}}\left(G_{\alpha}^{i} \cap P\right)$, es decir, el subespacio $A_{i}^{\prime}$ es unión de subconjuntos $G_{\delta}$ del compacto $P$ para cada $i \leq n$. De la igualdad

$$
\bigcup_{i=1}^{n} A_{i}^{\prime}=\bigcup_{i=1}^{n}\left(A_{i} \bigcap P\right)=P \bigcap\left(\bigcup_{i=1}^{n} A_{i}\right)=X \bigcap P=X \bigcap\left(\bigcap_{i=1}^{n}{\overline{A_{i}}}^{\beta X}\right)=\bigcap_{i=1}^{n} \overline{A_{i}}
$$

se desprende que $\bigcap_{i=1}^{n} \overline{A_{i}}$ es unión de conjuntos $G_{\delta}$ en $P$ y por lo tanto es de tipo puntual numerable.

2.4. Teorema. Sea $Y_{1} \times Y_{2} \times \cdots \times Y_{n}=X_{1} \cup X_{2} \cup \ldots \cup X_{n}$ donde $X_{i}$ es de tipo puntual numerable para toda $i \in\{1,2, \ldots, n\}$. Luego, existe $i_{0}$ tal que el espacio $Y_{i_{0}}$ es de tipo puntual numerable.

Demostración. Procederemos por inducción. El caso $n=1$ es claro. Supongamos que nuestro teorema se demostró para toda $m<n$.

Supongamos que $Y_{j}$ no es de tipo puntual numerable para toda $j \leq n-1$. Luego, para cada $j \leq n-1$ el espacio $Y_{j}$ no es localmente de tipo puntual numerable, es decir, existen $y_{j} \in Y_{j}$ tales que cualquier $U \in \mathcal{T}\left(y_{j}, Y_{j}\right)$ no es de tipo puntual numerable con $j \leq n-1$.

Consideremos el subespacio de tipo puntual numerable $F=\bigcap_{i=1}^{n} \overline{X_{i}}$ (lema 2.3) y el cerrado $Y=\left\{\left(y_{1}, y_{2}, \ldots, y_{n-1}, y\right): y \in Y_{n}\right\}$. Supongamos que $Y-F \neq \emptyset$. Tomemos un $z \in Y-F$. Luego $z=\left(y_{1}, y_{2}, \ldots, y_{n-1}, y_{n}\right)$ para algún $y_{n} \in Y_{n}$. Dado que $z \notin F$ existen $j_{0}$ y $U_{j} \in \mathcal{T}\left(y_{j}, Y_{j}\right)$ para todo $j \leq n$ tales que $V \cap X_{j_{0}}=\emptyset$ donde $V=U_{1} \times U_{2} \times \cdots \times U_{n}$. Sin pérdida de generalidad podemos suponer que $j_{0}=1$. Puesto que $V \subset X_{2} \cup X_{3} \cup \ldots \cup X_{n}$, obtenemos $V=X_{2}^{\prime} \cup X_{3}^{\prime} \cup \ldots \cup X_{n}^{\prime}$ donde $X_{j}^{\prime}=$ $X_{j} \cap V$ es de tipo puntual numerable. Sea $V^{\prime}=U_{1} \times U_{2} \times \cdots \times U_{n-1}$. Como $V^{\prime} \times\left\{y_{n}\right\}$ es un subespacio cerrado de $V$, se desprende que $V^{\prime} \times\left\{y_{n}\right\}=X_{2}^{\prime \prime} \cup X_{3}^{\prime \prime} \cup \ldots \cup X_{n}^{\prime \prime}$ donde $X_{j}^{\prime \prime}=X_{j}^{\prime} \cap\left(V^{\prime} \times\left\{y_{n}\right\}\right)$ es de tipo puntual numerable con $2 \leq j \leq n$. Dado que $V^{\prime} \times\left\{y_{n}\right\}$ y $V^{\prime}$ son homeomorfos, obtenemos $V^{\prime}=X_{2}^{\prime \prime \prime} \cup X_{3}^{\prime \prime \prime} \cup \ldots \cup X_{n}^{\prime \prime \prime}$ de tal 
forma que cada $X_{j}^{\prime \prime \prime}$ es de tipo puntual numerable. Por hipótesis inductiva, existe $i_{0} \in\{1,2, \ldots, n-1\}$ tal que $U_{i_{0}} \ni y_{i_{0}}$ es de tipo puntual numerable, lo cual es una contradicción.

Por lo tanto $Y \subset F$. En virtud de 2.2 el subespacio $Y$ es de tipo puntual numerable. Por ser $Y$ y $Y_{n}$ homeomorfos, se sigue que $Y_{n}$ es de tipo puntual numerable.

2.5. Corolario. Sea $X^{n}=X_{1} \cup X_{2} \cup \ldots \cup X_{n}$ donde $X_{i}$ es de tipo puntual numerable para toda $i \in\{1,2, \ldots, n\}$. Luego, el espacio $X$ es de tipo puntual numerable.

2.6. Corolario. Si $X^{n}=X_{1} \cup X_{2} \cup \ldots \cup X_{n}$ donde $X_{i}$ es localmente Čech completo para toda $i \leq n$, entonces el espacio $X$ es de tipo puntual numerable.

Demostración. Por el corolario 2.5, es suficiente demostrar que todo espacio localmente Čech completo es de tipo puntual numerable. Sea $X$ un espacio localmente Čech completo y $x \in X$. Tomemos un abierto Čech completo $U \ni x$. La proposición 1.25 garantiza la existencia de un compacto $K$ tal que $x \in K \subset U$ y $\chi(K, U) \leq \omega$. Luego, $\chi(K, X) \leq \omega$ y por lo tanto $X$ es de tipo puntual numerable.

En el siguiente ejemplo se establece que la Čech completitud no es aditiva.

2.7. Ejemplo. Existe un espacio no Čech completo que es la unión de dos subespacios Čech completos.

Demostración. Sea $X$ un espacio discreto y no numerable. Consideremos un punto $* \notin X$ y el conjunto $L(X)=X \cup\{*\}$. Definamos una topología en $L(X)$ de la siguiente forma: $\exp (X) \subset \mathcal{T}(L(X))$ y $\mathcal{T}(*, L(X))=\{\{*\} \cup(X-A):|A| \leq$ $\omega\}$. El espacio $(L(X), \mathcal{T}(L(X)))$ recibe el nombre de Extensión de Lindelöf de $X$. Claramente, los subespacios $X$ y $\{*\}$ de $L(X)$ son Cech completos. Probemos que $L(X)$ no es Čech completo. De acuerdo a la proposición 1.25, es suficiente verificar que $L(X)$ no es de tipo puntual numerable. Supóngase que existe un compacto $K \subset L(X)$ tal que $* \in K$ y $\chi(K, L(X)) \leq \omega$. Notemos que $K$ es finito, pues de lo contrario, si $|K| \geq \omega$ entonces la familia $\{\{k\}: k \in K-\{*\}\} \cup\{(X-K) \cup\{*\}\}$ es una cubierta abierta de $K$ que no posee subcubierta finita, lo cual contradice que $K$ sea compacto. Luego, podemos suponer sin pérdida de generalidad que $K=\{*\}$. Tomemos $\left\{U_{n}\right\}_{n \in \mathbb{N}} \subset \mathcal{T}(L(X))$ una base para $*$. Para cada $n \in \mathbb{N}$, sea $U_{n}=$ $\{*\} \cup\left(X-V_{n}\right)$ donde $\left|V_{n}\right| \leq \omega$. Dado que $\bigcap_{n \in \mathbb{N}} U_{n}=\{*\} \cup\left(X-\bigcup_{n \in \mathbb{N}} V_{n}\right)$ y como $\left|\bigcup_{n \in \mathbb{N}} V_{n}\right| \leq \omega$, obtenemos que $\bigcap_{n \in \mathbb{N}} U_{n} \in \mathcal{T}(*, L(X))$. De aquí se desprende que $\left|\bigcap_{n \in \mathbb{N}} U_{n}\right|>\omega$, lo cual contradice la igualdad $\{*\}=\bigcap_{n \in \mathbb{N}} U_{n}$.

Como se muestra en este ejemplo, el espacio $L(X)$ no es de tipo puntual numerable y es la unión de dos subespacios de tipo puntual numerable, de modo que si en el teorema 2.4 reemplazamos la hipótesis $\Pi_{i=1}^{n} Y_{i}=\bigcup_{i=1}^{n} X_{i}$ por $\prod_{i=1}^{n} Y_{i}=\bigcup_{i=1}^{m} X_{i}$ con $m>n$, entonces la conclusión es falsa. Observemos también que $L(X) \times\{*\}=$ 
$(X \times\{*\}) \cup\{(*, *)\}$ y dado que los subespacios $X \times\{*\}$ y $\{(*, *)\}$ son de tipo puntual numerable, en 2.4 tampoco se puede establecer que todos los espacios $Y_{i}$ sean de tipo puntual numerable.

Sea $X$ un espacio topológico. Una familia $\gamma \subset \exp (X)-\{\emptyset\}$ se llama localmente finita, si para todo $x \in X$ existe un $U \in \mathcal{T}(x, X)$ tal que $U$ intersecta sólo un número finito de elementos de $\gamma$. La familia $\gamma$ se llama puntualmente finita si la colección $\gamma_{x}=\{V \in \gamma: x \in V\}$ es finita para todo $x \in X$. En el ejemplo 1.34 proporcionamos un espacio no Čech completo con una cubierta abierta y puntualmente finita de subespacios Čech completos.

2.8. Teorema. Si un espacio $X$ tiene una cubierta abierta localmente finita $\mathcal{U}=$ $\left\{U_{t}\right\}_{t \in T}$ tal que $U_{t}$ es Čech completo para todo $t \in T$, entonces el espacio $X$ es Čech completo.

Demostración. Sea $U_{t}=X \cap V_{t}$ donde $V_{t} \in \mathcal{T}(\beta X), t \in T$. Consideremos la familia $\mu=\left\{V_{t}\right\}_{t \in T}$ y el conjunto

$$
V=\{x \in \beta X: \mu \text { es localmente finita en } x\} .
$$

El subespacio $V$ satisface las siguientes condiciones

(i) $V \in \mathcal{T}(\beta X)$;

(ii) $X \subset V$.

Demostremos (i). Dado un $y \in V$, existe $W \in \mathcal{T}(y, \beta X)$ tal que

$$
\left|\left\{t \in T: V_{t} \cap W \neq \emptyset\right\}\right|<\omega
$$

Afirmamos que $W \subset V$. Sea $z \in W$. Entonces $W \in \mathcal{T}(z, \beta X)$ y se cumple (1) por lo cual $z \in V$.

Para verificar la parte (ii), tomemos un $x \in X$. Sabemos que existe $W \in \mathcal{T}(x, X)$ tal que

$$
\left|\left\{t \in T: W \cap U_{t} \neq \emptyset\right\}\right|<\omega
$$

Tomemos un $G \in \mathcal{T}(x, \beta X)$ para el cual $W=X \cap G$. Así que

$$
\begin{aligned}
& \left|\left\{t \in T: G \cap V_{t} \neq \emptyset\right\}\right|=\left|\left\{t \in T: X \cap G \cap V_{t} \neq \emptyset\right\}\right|= \\
& \left|\left\{t \in T: G \cap U_{t} \neq \emptyset\right\}\right|=\left|\left\{t \in T: W \cap U_{t} \neq \emptyset\right\}\right|<\omega
\end{aligned}
$$

Por lo tanto $x \in V$.

Ahora ya estamos listos para probar que $X$ es Čech completo. Observemos primero que para todo $t \in T$ se tiene que $U_{t}=\bigcap_{n \in \mathbb{N}} V_{t}^{n}$, donde $V_{t}^{n} \in \mathcal{T}\left(V_{t}\right)$, para todo $n \in \mathbb{N}$, debido a que $U_{t}$ es Čech completo y $\overline{U_{t}}=\overline{X \cap V_{t}}=\overline{V_{t}}$ para cada $t \in T$. Sin pérdida de generalidad podemos suponer que para cada $t \in T$ se cumple

$$
V_{t}^{n+1} \subset V_{t}^{n}, \quad n \in \mathbb{N} \text {. }
$$


Sea $V_{n}=\bigcup_{t \in T} V_{t}^{n}$ para todo $n \in \mathbb{N}$. Probemos que $X=\bigcap_{n \in \mathbb{N}} V_{n}$.

Sea $n \in \mathbb{N}$. De $U_{t} \subset V_{t}^{n}$, se sigue que $X \subset \bigcup_{t \in T} V_{t}^{n}$, de donde $X \subset V_{n}$. Ésto implica que $X \subset \bigcap_{n \in \mathbf{N}} V_{n}$.

Ahora demostremos que $\bigcap_{n \in \mathbb{N}} V_{n} \subset X$. Sea $z \in \bigcap_{n \in \mathbb{N}} V_{n}$. Como $z \in V_{n}$ para todo $n \in \mathbb{N}$ existe $t_{n} \in T$ tal que $z \in V_{t_{n}}^{n}$ para cada $n \in \mathbb{N}$. Tenemos que $V_{t_{n}}^{n} \subset V_{t_{n}} \subset V$ y por lo tanto $z \in V$, lo cual implica que existe $W \in \mathcal{T}(z, V)$ tal que

$$
\left|\left\{t \in T: W \cap V_{t} \neq \emptyset\right\}\right|<\omega .
$$

Como $W \cap V_{t_{n}}^{n} \neq \emptyset$, tenemos que $W \cap V_{t_{n}} \neq \emptyset$ y por lo tanto el conjunto $\left\{t_{n} \in T\right.$ : $n \in \mathbb{N}\}$ es finito. De aquí se sigue que existe $n_{0} \in \mathbb{N}$ tal que para todo $m \geq n_{0}$, se tiene que $t_{m}=t_{n_{0}}$. De modo que $z \in V_{t_{n_{0}}}^{m}$ para todo $m \geq n_{0}$. La condición (2) implica que $z \in \bigcap_{m \in \mathbb{N}} V_{t_{n_{0}}}^{m}=U_{t_{n_{0}}} \subset X$.

Dado que toda cubierta finita es localmente finita, obtenemos como consecuencia inmediata de este resultado, el siguiente Corolario.

2.9. Corolario. Si $X=A_{1} \cup A_{2} \cup \ldots \cup A_{n}$ donde $A_{i}$ es abierto y Čech completo para $1 \leq i \leq n$, entonces $X$ es Čech completo.

2.10. Lema. Si $X=A_{1} \cup A_{2} \cup \ldots \cup A_{n}$ donde $A_{i}$ es Čech completo para todo $i \in\{1,2, \ldots, n\}$, entonces $\bigcap_{i=1}^{n} \overline{A_{i}}$ es Čech completo.

Demostración. Es suficiente demostrar que $\bigcap_{i=1}^{n} \overline{A_{i}}$ es un subconjunto $G_{\delta}$ del compacto $P=\bigcap_{i=1}^{n}{\overline{A_{i}}}^{\beta X}$.

Como cada subespacio $A_{i}$ es Čech completo para $1 \leq i \leq n$, deducimos que $\bar{A}_{i}^{\beta X}-A_{i}=\bigcup_{j \in \mathbb{N}} F_{j}$ donde $F_{j}$ es compacto para todo $j \in \mathbb{N}$. Sea $A_{i}^{\prime}=A_{i} \cap P$. Luego $P-A_{i}^{\prime}=P-A_{i}=P \bigcap\left({\overline{A_{i}}}^{\beta X}-A_{i}\right)=P \bigcap\left(\bigcup_{j \in \mathbb{N}} F_{j}\right)=\bigcup_{j \in \mathbb{N}}\left(F_{j} \cap P\right)$.

De aquí se desprende que $P-A_{i}^{\prime}$ es $F_{\sigma}$ en $P$ y por lo tanto $A_{i}^{\prime}$ es un subconjunto $G_{\delta}$ de $P$. Como

$\bigcup_{i=1}^{n} A_{i}^{\prime}=\bigcup_{i=1}^{n}\left(A_{i} \bigcap P\right)=P \bigcap\left(\bigcup_{i=1}^{n} A_{i}\right)=X \bigcap P=X \bigcap\left(\bigcap_{i=1}^{n}{\overline{A_{i}}}^{\beta X}\right)=\bigcap_{i=1}^{n} \overline{A_{i}}$ tenemos que $\bigcap_{i=1}^{n} \overline{A_{i}}$ es unión finita de conjuntos $G_{\delta}$ en $P$ y por lo tanto es $G_{\delta}$ en $P$.

2.11. Corolario. Si $X=A_{1} \cup A_{2} \cup \ldots \cup A_{n}$ donde $A_{i}$ es Čech completo y denso en $X$ para todo $i \in\{1,2, \ldots, n\}$, entonces $X$ es un espacio Čech completo.

2.12. Teorema. Si $X=A_{1} \cup A_{2} \cup \ldots \cup A_{n}$ donde $A_{i}$ es localmente Čech completo para todo $i \in\{1,2, \ldots, n\}$, entonces $X$ contiene un subespacio abierto no vacío $y$ Čech completo.

Demostración. Usemos la inducción sobre $n$. Si $n=1$ entonces $X$ es localmente Čech completo. Tomemos un $x \in X$. Como $X$ es localmente Čech completo, existe 
un abierto $U \subset X$ tal que $x \in U$ y $U$ es Čech completo. Es claro que $U \neq \emptyset$, así que en este caso tenemos el conjunto prometido. Supongamos que nuestro teorema se probó para todo $n<k$ y $X=A_{1} \cup A_{2} \cdots \cup A_{k}$, donde cada $A_{i}$ es localmente Čech completo. Si $\overline{A_{i_{0}}} \neq X$ para algún $i_{o} \leq k$ entonces $Y=X-\overline{A_{i_{0}}}$ es abierto y no vacío. Como $A_{i} \cap Y$ es localmente Čech completo, el espacio $Y$ se representa como unión de $\leq k-1$ subespacios localmente Čech completos. La hipótesis inductiva nos garantiza la existencia de un $U \in \mathcal{T}^{*}(Y)$ que es Čech completo. Es evidente que $U \in \mathcal{T}^{*}(X)$, así que en este caso queda demostrado el teorema.

Supongamos ahora que $\overline{A_{i_{0}}}=X$ para todo $i \leq k$. Notemos que en el caso de $n=1$ probamos que cada espacio no vacío localmente Čech completo tiene un subespacio abierto no vacío Čech completo. Sea $W_{1} \in \mathcal{T}^{*}\left(A_{1}\right)$ un espacio Čech completo. Tomemos un $U_{1} \in \mathcal{T}(X)$ con $U_{1} \cap A_{1}=W_{1}$. Supongamos que tenemos $U_{1}, U_{2}, \ldots, U_{m} \in \mathcal{T}^{*}(X)$ tales que $U_{i} \cap A_{j}$ es Čech completo para todo $j \leq i \mathrm{y}$ todo $i \leq m$. Como $U_{m} \cap A_{m+1} \neq \emptyset$ es localmente Čech completo, existe $W_{m+1} \in$ $\mathcal{T}^{*}\left(U_{m} \cap A_{m+1}\right)$ que es Čech completo. Tomemos cualquier $U_{m+1} \in \mathcal{T}\left(U_{m}\right)$ tal que $U_{m+1} \cap A_{m+1}=W_{m+1}$. Entonces $U_{m+1} \cap A_{m+1}$ es Čech completo por construcción. Si $i<m+1$ entonces $U_{m+1} \cap A_{i}$ es un subespacio abierto de $U_{m} \cap A_{i}$ el cual es Čech completo. Por lo tanto $U_{m+1} \cap A_{i}$ es Čech completo para todo $i \leq m+1$. Esta construcción inductiva hace posible realizar $k$ pasos construyendo así el conjunto $U_{k} \in \mathcal{T}^{*}(X)$. De aquí y del corolario 2.11 vemos que $U_{k}$ es un subconjunto abierto Čech completo, ya que

$U_{k}=\left(U_{k} \cap A_{1}\right) \cup\left(U_{k} \cap A_{2}\right) \cup \cdots \cup\left(U_{k} \cap A_{n}\right) \quad$ y $\quad \overline{U_{k} \cap A_{i}}=\overline{U_{k}} \quad$ para $\quad 1 \leq i \leq k$.

2.13. Corolario. Si $X=A_{1} \cup A_{2} \cup \ldots \cup A_{n}$ donde $A_{i}$ es localmente Čech completo para todo $i \in\{1,2, \ldots, n\}$, entonces existe un abierto Čech completo y denso en $X$.

Demostración. Sea $U \in \mathcal{T}^{*}(X)$, luego $U=\left(A_{1} \cap U\right) \cup \ldots \cup\left(A_{n} \cap U\right)$ donde $A_{i} \cap U$ es localmente Čech completo para todo $1 \leq i \leq n$. En virtud del teorema anterior, existe un $V \in \mathcal{T}^{*}(X)$, tal que $V \subset U$ y $V$ es Čech completo. Por lo tanto el conjunto

$$
\mathcal{P}=\left\{V \in \mathcal{T}^{*}(X): V \text { es Čech completo }\right\}
$$

es una $\pi$-base en $X$. Consideremos la familia $\gamma=\{\delta \subset \mathcal{P}: \delta$ es ajena $\}$. Para cualesquiera $\delta_{1}, \delta_{2} \in \gamma$, decimos que $\delta_{1} \leq \delta_{2}$ si $\delta_{1} \subset \delta_{2}$. Es rutina verificar que $(\gamma, \leq)$ tiene cadenas acotadas. Tomemos una subfamilia maximal $\mu \subset \gamma$. Consideremos el abierto $G=\cup \mu$ que es Čech completo por ser homeomorfo a la suma discreta de los elementos de $\mu$ (teorema 1.20). Tenemos que $\bar{G}=X$, pues de lo contrario $X-\bar{G} \in \mathcal{T}^{*}(X)$ y por lo tanto existiría $U \in \mathcal{P}$ tal que $U \subset X-\bar{G}$. Si $\mu^{*}=\mu \cup\{U\}$, entonces $\mu \subset \mu^{*}$ y $\mu^{*}$ es ajena contradiciendo la maximalidad de $\mu$. 
2.14. Corolario. Si un espacio $X$ es la unión de una familia localmente finita de subespacios Čech completos, entonces existe un subespacio abierto no vacío y Čech completo de $X$.

2.15. Corolario. Si $X=A_{1} \cup A_{2} \cup \ldots \cup A_{n}$ donde $A_{i}$ es Čech completo para $i \in$ $\{1,2 \ldots, n\}$, entonces en $X$ existe un subespacio abierto no vacío y $\breve{C}$ ech completo.

2.16. Corolario. Si $X=A_{1} \cup A_{2} \cup \ldots \cup A_{n}$ donde $A_{i}$ es casi Čech completo para todo $i \in\{1,2, \ldots, n\}$ entonces $X$ es casi Čech completo.

Demostración. Para cada $i=1,2, \ldots, n$ sean los subespacios Čech completos $B_{i} \subset A_{i}$ tales que $\overline{B_{i}}=\overline{A_{i}}$. Luego, $Y=\bigcup_{i=1}^{n} B_{i}$ es un subespacio denso de $X$. Por el corolario 2.13, existe un subespacio $Z$ Cech completo y denso en $Y$. Es claro que $Z$ también es denso en $X$.

La siguiente proposición afirma que la Čech completitud es finitamente aditiva en espacios metrizables. La demostración original fue bastante laboriosa, motivo por el cual presentamos aquí una demostración corta sugerida por el Dr. O. Okunev al enterarse del resultado.

2.17. Proposición. Sea $X$ un espacio metrizable. Si $X=A_{1} \cup A_{2} \cup \ldots \cup A_{n}$ donde $A_{i}$ es Čech completo para toda $i \leq n$, entonces $X$ es Čech completo.

Demostración. Consideremos la completación $\widetilde{X}$ del espacio $X$. Como $\widetilde{X}$ es un espacio métrico cada subespacio $A_{i}$ con $i \leq n$ es un subconjunto $G_{\delta}$ de $\widetilde{X}$. Por lo tanto, el espacio $X$ es una unión finita de subconjuntos $G_{\delta}$ de $\widetilde{X}$, así que $X$ es un subconjunto $G_{\delta}$ de $\widetilde{X}$. De aquí resulta que $X$ es Čech completo.

2.18. Lema. Si $X^{\omega}$ contiene un subespacio abierto, no vacío y Čech completo, entonces $X^{\omega}$ es Čech completo.

Demostración. Tomemos un $U \in \mathcal{T}^{*}\left(X^{\omega}\right)$ Čech completo. Existe un elemento $B$ de la base canónica en $X^{\omega}$ tal que $B \subset U$. Sea $B=\left\{x \in X^{\omega}: x\left(\alpha_{i}\right) \in B_{i}, i=\right.$ $1,2 \ldots, n\}$ con $n \in \mathbb{N}$ y $B_{i} \in \mathcal{T}^{*}(X)$ para $i \leq n$. Fijémos un $x \in X^{A}$ donde $A=\left\{\alpha_{1}, \alpha_{2}, \ldots, \alpha_{n}\right\}$ y $x\left(\alpha_{i}\right) \in B_{i}$, con $i \leq n$. Entonces $Y=\{x\} \times X^{\omega-A}$ es un subespacio cerrado de $X^{\omega}$ tal que $Y \subset B \subset U$. De aquí resulta que $Y$ es cerrado en $U$ y dado que $U$ es Čech completo, el subespacio $Y$ también lo es. Como $Y$ es homeomorfo a $X^{\omega}$, tenemos que $X^{\omega}$ es Čech completo.

Dado que la Čech completitud es una propiedad que se hereda a subconjuntos cerrados, la demostración anterior es esencialmente la misma si reemplazamos $X^{\omega}$ por un producto $\Pi_{\lambda<\alpha} X_{\lambda}$. Así que, tenemos una generalización del lema 2.18: 
(P1) Sean $P$ una propiedad topológica que es hereditaria respecto a subconjuntos cerrados y $\alpha$ un cardinal infinito. Para cada ordinal $\lambda<\alpha$ sea $X_{\lambda}$ un espacio topológico con $\left|X_{\lambda}\right|>1$. Si el producto $X=\Pi_{\lambda<\alpha} X_{\lambda}$ contiene un subconjunto abierto que satisface $\mathcal{P}$ entonces $X$ también posee la propiedad $\mathcal{P}$.

2.19. Corolario. Si $X^{\omega}$ es localmente Čech completo entonces $X^{\omega}$ es Čech completo.

Aplicando 2.12 y 2.18 obtenemos

2.20. Teorema. Si $X^{\omega}=A_{1} \cup A_{2} \cup \ldots \cup A_{n}$ donde $A_{i}$ es localmente Čech completo para todo $i \in\{1,2, \ldots, n\}$, entonces $X$ es un espacio Čech completo.

Otra forma de llegar a este resultado, es obtenerlo como caso particular de la propiedad siguiente, la cual se sigue de 2.12 y (P1)

(P2) Sean $\alpha, \lambda$ y $X_{\lambda}$ como en (P1). Si el producto $X=\Pi_{\lambda<\alpha} X_{\lambda}$ satisface $X=A_{1} \cup A_{2} \cup \ldots \cup A_{n}$ donde $A_{i}$ es localmente Čech completo para todo $i \in\{1,2, \ldots, n\}$, entonces $X$ es Čech completo y cada $X_{\lambda}$ también lo es.

2.21. Corolario. Si $X^{\omega}=A_{1} \cup A_{2} \cup \ldots \cup A_{n}$ donde $A_{i}$ es Čech completo para $1 \leq i \leq n$ entonces $X$ es un espacio Čech completo.

2.22. Teorema. Si $X^{n}=A_{1} \cup A_{2} \cup \ldots \cup A_{n}$ donde $A_{i}$ es Čech completo para $1 \leq i \leq n$ entonces $X$ es un espacio localmente Čech completo.

Demostración. Utilizaremos la inducción sobre $n$. Es evidente que el teorema es cierto para $n=1$. Supongamos que se estableció el teorema para todo $n \leq m-1 \mathrm{y}$ $X^{m}=A_{1} \cup A_{2} \cup \ldots \cup A_{m}$ donde cada $A_{i}$ es Čech completo. Tomemos $x_{0} \in X$. En virtud del lema 2.10 el subespacio $F=\bigcap_{i=1}^{m} \overline{A_{i}} \subset X^{m}$ es Čech completo. Fijémonos en el cerrado $Y=\left\{\left(x_{0}^{m-1}, y\right): y \in X\right\}$ donde $x_{0}^{m-1}=\left(x_{0}, x_{0}, \ldots, x_{0}\right) \in X^{m-1}$. Dividamos la prueba en dos casos. Si $Y \subset F$, se deduce que $Y$ es Čech completo. Como $Y$ es homeomorfo a $X$, el espacio $X$ es localmente Čech completo.

En el caso $Y-F \neq \emptyset$ tomemos un punto $\left(x_{0}^{n-1}, y\right) \in Y-F$. Existen $U \in \mathcal{T}\left(x_{0}, X\right)$, $V \in \mathcal{T}(y, X)$ y $i_{0} \in\{1,2, \ldots, m\}$ tales que $\left(U^{m-1} \times V\right) \bigcap A_{i_{0}}=\emptyset$. Sin pérdida de generalidad podemos suponer que $i_{0}=1$. Puesto que $U^{m-1} \times\{y\} \subset U^{m-1} \times V$ y $U^{m-1} \times\{y\}$ es homeomorfo a $U^{m-1}$, podemos afirmar que $U^{m-1}=A_{2}^{\prime} \cup A_{3}^{\prime} \cup \ldots \cup A_{m}^{\prime}$ donde $A_{j}^{\prime}$ es Čech completo para $2 \leq j \leq m$. Por la hipótesis inductiva el subespacio $U$ es localmente Čech completo. Siendo $x_{0}$ un punto arbitrario, el espacio $X$ es localmente Čech completo. 


\section{La no aditividad de la Čech completitud en potencias finitas}

¿En el teorema anterior se puede concluir que el espacio $X$ es Čech completo? La respuesta es negativa. En el ejemplo 2.26 mostraremos que hay modelos de ZFC en los cuales la Čech completitud no es aditiva en potencias finitas. Para ello necesitaremos algunos resultados previos.

2.23. Definición. Un espacio $X$ es llamado un espacio escalera sobre un subconjunto $S$ de un ordinal $\kappa$ si

(a) $X=\kappa$;

(b) Los puntos de $\kappa-S$ son aislados;

(c) Para cada $\alpha \in S$, existe un subconjunto cofinal $L_{\alpha} \subset \alpha-S$ tal que

$$
\left\{(\beta, \alpha] \cap\left(L_{\alpha} \cup\{\alpha\}\right): \beta<\alpha\right\}
$$

es una base en $\alpha$.

El siguiente lema [BGT] nos será de gran utilidad.

2.24. Lema. Sea $X$ un espacio escalera sobre un subconjunto $S$ de un ordinal $\kappa$. Entonces, para cualquier $n \in \mathbb{N}$ tenemos que $X^{n}=A_{0} \cup A_{1}$ donde $A_{0}$ y $A_{1}$ son la unión discreta de subespacios de cardinalidad menor que $\kappa$.

Demostración. Sean

$$
A_{0}=\left\{\left(\alpha_{i}\right)_{i<n} \in X^{n}: \max \left\{\alpha_{i}: i<n\right\} \in S\right\} \quad \text { y } \quad A_{1}=X^{n}-A_{0} .
$$

Para cada $\delta<\kappa$, consideremos el conjunto

$$
M_{\delta}=\left\{\left(\alpha_{i}\right)_{i<n} \in X^{n}: \max \left\{\alpha_{i}: i<n\right\}=\delta\right\} .
$$

Afirmamos que $M_{\delta}$ es un subconjunto abierto-cerrado de $A_{0}$ si $\delta \in S$. Sea $\left(\alpha_{i}\right)_{i<n} \in$ $M_{\delta} \subset A_{0}$. Tomemos $\beta=\max \left\{\alpha_{i}: i<n, \alpha_{i}<\delta\right\}$. Para cada $i<n$ escojamos una vecindad básica $U_{i}$ de $\alpha_{i}$ para la cual $\alpha_{i}=\sup U_{i}$ y si $\alpha_{i}=\delta$ entonces $\inf U_{i}>\beta$. El abierto $V=A_{0} \cap\left(U_{0} \times U_{1} \times \cdots \times U_{n-1}\right) \ni\left(\alpha_{i}\right)_{i<n}$ verifica $V \subset M_{\delta}$. En efecto, sea $\left(\gamma_{i}\right)_{i<n} \in V$. Tomemos $\gamma^{*}=\max \gamma_{i}$. Luego, $\gamma^{*} \leq \delta$. Tenemos que $\gamma^{*} \in U_{i} \cap S$ para algún $i<n$. Ésto significa que $\gamma^{*}=\alpha_{i}$ y por lo tanto $\gamma^{*} \leq \beta$ ó $\gamma^{*}=\delta$. Como $\gamma^{*}$ es la coordenada máxima, tenemos que $\gamma^{*}>\beta$ y por lo tanto $\gamma^{*}=\delta$, lo que implica $\left(\gamma_{i}\right)_{i<n} \in M_{\delta}$. De modo que $M_{\delta}$ es un subconjunto abierto de $A_{0}$. Ahora verifiquemos que $M_{\delta}$ es cerrado en $A_{0}$. Sea $\left(\gamma_{i}\right)_{i<n} \in A_{0}-M_{\delta}$. Tenemos que $\left(\gamma_{i}\right)_{i<n} \in M_{\gamma^{*}}$ donde $\gamma^{*}=\max \gamma_{i} \neq \delta$. Dado que $\gamma^{*} \in S$, el conjunto $M_{\gamma^{*}}$ es abierto en $A_{0}$. Como $\left(\gamma_{i}\right)_{i<n} \in M_{\gamma^{*}} \subset A_{0}-M_{\delta}$, se desprende que $M_{\delta}$ es cerrado en $A_{0}$.

Ahora supongamos que $\delta \notin S$ y probemos que $M_{\delta}$ es un subconjunto abierto-cerrado de $A_{1}$. Sea $\left(\alpha_{i}\right)_{i<n} \in M_{\delta} \subset A_{1}$. Para cada $i<n$, tomemos una vecindad básica 
$U_{i}$ de $\alpha_{i}$ tal que $\sup U_{i}=\alpha_{i}$ y si $\alpha_{i}=\delta$ entonces $U_{i}=\{\delta\}$. Es inmediato que $\left(\alpha_{i}\right)_{i<n} \in A_{1} \cap\left(U_{0} \times U_{1} \times \cdots \times U_{n-1}\right) \subset M_{\delta}$ y por lo tanto $M_{\delta}$ es abierto en $A_{1}$. De forma similar al caso $\delta \in S$, se prueba que $M_{\delta}$ también es cerrado en $A_{1}$.

Por lo anterior, deducimos que $A_{0}=\bigoplus_{\delta \in S} M_{\delta}$ y $A_{1}=\bigoplus_{\delta \notin S} M_{\delta}$. Como $\left|M_{\delta}\right|<\kappa$ para todo $\delta<\kappa$, se cumple lo pedido.

Un conjunto $C \subset \omega_{1}$ se llama $c l u b$ si es cerrado y no acotado en $\omega_{1}$. Decimos que $S \subset \omega_{1}$ es estacionario si $S \cap C \neq \emptyset$ para todo club $C$. Denotemos por $L$ el conjunto de los ordinales límite en $\omega_{1}$, es decir, $L=\left\{\alpha \in \omega_{1}: \alpha=\lim \alpha\right\}$. Recordemos el axioma $\diamond^{+}$que es consistente con ZFC, ver [Ku, definición 7.9]:

Existe una sucesión $\left\{\mathcal{A}_{\alpha}: \alpha<\omega_{1}\right\}$, donde $\mathcal{A}_{\alpha}$ es una familia numerable de subconjuntos de $\alpha$, para la cual dado cualquier $A \subset \omega_{1}$ existe un club $C \subset \omega_{1}$ tal que para todo $\alpha \in C$ se cumplen $A \cap \alpha \in \mathcal{A}_{\alpha}$ y $C \cap \alpha \in \mathcal{A}_{\alpha}$.

2.25. Lema. Existen subconjuntos $E, G \subset L$, tales que

(a) E y $G$ son estacionarios;

(b) $E \cap G=\emptyset$;

(c) Para todo $\alpha \in G$ existe $\left\{\alpha_{n}\right\}_{n \in \omega} \subset E$ con $\alpha_{n+1}>\alpha_{n}$ para todo $n \in \omega y$ $\alpha_{n} \longrightarrow \alpha$.

Demostración. De [Ku, corolario 6.12] sabemos que existen conjuntos estacionarios y ajenos $E, G^{\prime} \subset L$. Sea

$P=\left\{\alpha \in \omega_{1}\right.$ : existe $\left\{\alpha_{n}\right\}_{n \in \omega} \subset E$ con $\alpha_{n+1}>\alpha_{n}$ para todo $n \in \omega$ y $\left.\alpha_{n} \longrightarrow \alpha\right\}$.

Probemos que $P$ es club. Dado $\alpha \in \omega_{1}$, notemos que $E$ es no acotado, y por lo tanto existe una sucesión creciente $\left\{\alpha_{n}\right\}_{n \in \omega} \subset E$ con $\alpha_{n}>\alpha$ para todo $n \in \omega$. La sucesión $\left\{\alpha_{n}\right\}_{n \in \omega}$ converge, digamos a un $\beta<\omega_{1}$. Es claro que $\beta>\alpha$ y $\beta \in P$. Por lo tanto $P$ es un conjunto no acotado. Para verificar que $P$ es cerrado, consideremos $\left\{\alpha_{n}\right\}_{n \in \omega} \subset P$ con $\alpha_{n+1}>\alpha_{n}$ para todo $n$ y $\alpha_{n} \longrightarrow \beta$. Luego, para cada $n \in \omega$ existe $\left\{\beta_{i}^{n}\right\}_{i \in \omega} \subset E$ con $\beta_{i}^{n} \longrightarrow \alpha_{n}$. Sea $\gamma_{n} \in\left(\alpha_{n-1}, \alpha_{n}\right) \cap\left\{\beta_{i}^{n}\right\}_{i \in \omega}$. De aquí resulta que $\left\{\gamma_{n}\right\}_{n \in \omega} \subset E, \gamma_{n+1}>\gamma_{n}$ para todo $n$ y $\gamma_{n} \longrightarrow \beta$, por lo cual $\beta \in P$. Continuando con nuestra demostración, observemos que el conjunto $G=G^{\prime} \cap P$ es estacionario, de tal manera que $E$ y $G$ cumplen (a), (b) y (c).

2.26. Ejemplo. Bajo el axioma $\diamond^{+}$existe un espacio $X$ que no es $\breve{C}$ ech completo $y$ tal que para todo $n \in \omega$ se tiene que $X^{n}=A_{0} \cup A_{1}$, donde $A_{0}$ y $A_{1}$ son subespacios Čech completos.

Demostración. Consideremos los conjuntos $E$ y $G$ dados en el lema anterior. Dado $\alpha \in G$, construyamos un conjunto $A_{\alpha} \subset \alpha-G$. Como $\alpha$ es un ordinal límite, tenemos que existe una sucesión creciente $\left\{\beta_{n}\right\}_{n \in \omega} \subset \omega_{1}-L$ convergente a $\alpha$. Tomemos la familia $\mathcal{A}_{\alpha}=\left\{A_{\alpha}^{n}\right\}_{n \in \omega}$ dada por el axioma $\diamond^{+}$. 
Para cada $n \in \omega$ construyamos una sucesión $s_{n}^{\alpha}$ de la siguiente forma: si $A_{n}^{\alpha} \cap E$ es cofinal en $\alpha$, entonces $s_{n}^{\alpha}$ es una sucesión de elementos de $A_{n}^{\alpha} \cap E \cap\left(\beta_{n}, \alpha\right)$ convergente a $\alpha$; en caso contrario, tomemos $s_{n}^{\alpha}=\emptyset$. Sea $s(\alpha)=\bigcup_{n \in \omega} s_{n}^{\alpha}$. Observemos que si $s(\alpha) \neq \emptyset$ entonces $s(\alpha) \longrightarrow \alpha$. En efecto, sea $\beta<\alpha$. Como $\beta_{n}>\beta$ para algún $n \in \omega$, se puede suponer que $\beta=\beta_{n}$. Por nuestra construcción $s_{i}^{\alpha} \subset\left(\beta_{i}, \alpha\right]$. Como $\left\{\beta_{i}\right\}_{i \in \omega}$ es creciente, tenemos que $s_{i}^{\alpha} \subset\left(\beta_{i}, \alpha\right] \subset(\beta, \alpha]$ para todo $i>n$. Ésto nos conduce a que solamente los puntos de $s_{j}^{\alpha}$ para $0 \leq j \leq n$ pueden no pertenecer a $\left(\beta_{n}, \alpha\right]$. Como todas las sucesiones $s_{i}^{\alpha}$ para $i \leq n$, son vacías ó convergen a $\alpha$, se sigue que cada una de ellas, y por lo tanto su unión, puede tener a lo más un número finito de elementos en $\omega_{1}-\left(\beta_{n}, \alpha\right]$. De aquí concluimos que $s(\alpha) \longrightarrow \alpha$. Denotemos $s(\alpha)=\left\{s_{k}: k \in \omega, s_{k+1}>s_{k}\right\}$.

Dado $\beta \in E$, consideremos la sucesión $\gamma(\beta)=\left\{\gamma_{n}(\beta): n \in \omega\right\} \subset \omega_{1}-L$, con $\gamma_{n+1}(\beta)>\gamma_{n}(\beta)$ para todo $n$ y $\gamma_{n}(\beta) \longrightarrow \beta$. Como $s(\alpha) \subset E$, para cada $k \in \omega$ existe $m_{k} \in \omega$ tal que $\gamma_{l}\left(s_{k}\right) \in\left(s_{k-1}, s_{k}\right)$ para todo $l \geq m_{k}$. Hagamos

$$
A_{\alpha}=\bigcup_{k \in \omega}\left\{\gamma_{l}\left(s_{k}\right): l \geq m_{k}\right\}
$$

Sea $X$ el espacio escalera del conjunto $G$ de $\omega_{1}$, cuya "escalera" en cada $\alpha \in G$ es el conjunto $A_{\alpha}$ dado en (3). Mostremos que $X$ es un espacio de Tychonoff, probando que cada vecindad básica $V=\left((\beta, \alpha] \cap A_{\alpha}\right) \cup\{\alpha\}$ de $\alpha \in G$ es un subespacio cerrado.

Sea $\delta \in \omega_{1}-V$. Consideremos los $\operatorname{casos} \delta>\alpha$ y $\delta<\alpha$.

En el primer caso, tenemos que $\delta$ pertenece al intervalo $U=(\alpha, \delta]$ y $U \cap V=\emptyset$. Cuando $\delta<\alpha$, si $\delta \notin G$ entonces $\{\delta\} \cap V=\emptyset$ y si $\delta \in G$ se sigue que $\delta \notin E$ de donde $\delta \notin s(\alpha)$. Sea $s_{-1}=0$. Elijamos $k \in \omega$ para el cual $\delta \in\left(s_{k-1}, s_{k}\right)$. Si $k=0$ tomemos $U=(0, \delta]$. Luego $U \cap V=\emptyset$. Consideremos el caso $k \geq 1$. Sea el conjunto $B=\left\{j \in \omega: s_{k-1}<\gamma_{j}\left(s_{k}\right)<\delta\right\}$. Notemos que $|B|<\omega$. Si $B=\emptyset$ tomemos $U=\left(s_{k-1}, \delta\right]$. Si $B \neq \emptyset$ sea $U=\left(\gamma_{j_{0}}\left(s_{k}\right), \delta\right]$, donde $j_{0}=\max B$. Para ambas posibilidades obtenemos que $U \cap V=\emptyset$. De lo anterior se desprende que $V$ es cerrado. Puesto que $X$ es un espacio de Hausdorff, concluimos que $X$ es de Tychonoff.

Supóngase que $X$ es Čech completo. Entonces existe una sucesión completa $\left\{\mathcal{U}_{n}\right\}_{n \in \omega}$ de cubiertas abiertas de $X$. Por la proposición1.17, podemos suponer que todo $U \in \mathcal{U}_{n}$ es igual a $\{\alpha\}$ si $\alpha \notin G$ ó es de la forma $\left((\beta, \alpha] \cap A_{\alpha}\right) \cup\{\alpha\}$ para el caso en que $\alpha \in G$. Además, puesto que para todo $\alpha \in X$, existe $U \in \mathcal{U}_{n}$ con $\alpha \in U$, se puede suponer que $\mathcal{U}_{n}=\left\{U_{\alpha}: \alpha \in \omega_{1}\right\}$ donde $U_{\alpha}=\{\alpha\}$ ó $U_{\alpha}=\left(\left(\beta_{n}^{\alpha}, \alpha\right] \cap A_{\alpha}\right) \cup\{\alpha\}$ $\operatorname{con} \beta_{n+1}^{\alpha}>\beta_{n}^{\alpha}$ para todo $n \in \omega$.

2.27. Definición. Sea $\alpha \in G$ y $U$ una vecindad básica de $\alpha$. Decimos que $\beta \in E$ casi pertenece a $U$ si existe $\gamma<\alpha$ tal que $U=\left((\gamma, \alpha] \cap A_{\alpha}\right) \cup\{\alpha\}$ y $\beta \in s(\alpha) \cap(\gamma, \alpha)$.

Afirmación. Existen $\beta \in E$ y $\left\{U_{n}\right\}_{n \in \omega}$ con $U_{n} \in \mathcal{U}_{n}$ para todo $n \in \omega$ tales que $\beta$ casi pertenece a $U_{n}$ para todo $n \in \omega$. 
Demostración. Supóngase lo contrario. Entonces para todo $\beta \in E$ existe $n_{\beta} \in \omega$ para el cual $\beta$ no casi pertenece a ningún $U \in \mathcal{U}_{n_{\beta}}$. Consideremos la función $\phi: E \rightarrow \omega$, dada por $\phi(\beta)=n_{\beta}$. Dado que $E=\bigcup \phi^{-1}\left(n_{\beta}\right)$, y por ser $E$ un conjunto estacionario, existe $\beta \in E$ para el cual $E^{\prime}=\phi^{-1}\left(n_{\beta}\right) \subset E$ es estacionario. Por lo tanto, $n_{\beta}=n_{\beta^{\prime}}=n$ para todos $\beta, \beta^{\prime} \in E^{\prime}$ y alguna $n \in \omega$. Sea

$P^{\prime}=\left\{\alpha \in \omega_{1}\right.$ : existe $\left\{\alpha_{n}\right\}_{n \in \omega} \subset E^{\prime}$ con $\alpha_{n+1}>\alpha_{n}$ para todo $n \in \omega$ y $\left.\alpha_{n} \longrightarrow \alpha\right\}$. De manera análoga que en la demostración del lema 2.25 para $P$, deducimos que $P^{\prime}$ es club. El axioma $\diamond^{+}$nos garantiza la existencia de un club $C$ tal que $E^{\prime} \cap \alpha \in \mathcal{A}_{\alpha}$ para todo $\alpha \in C$. De lo anterior se desprende que el $P^{\prime} \cap C$ es club, por lo que $P^{\prime} \cap C \cap G$ es un conjunto estacionario.

Sea $\alpha \in P^{\prime} \cap C \cap G$. Entonces $E^{\prime} \cap \alpha=A_{\alpha}^{k} \in \mathcal{A}_{\alpha}$ para algún $k \in \omega$. Dado que $\alpha \in P^{\prime}$, para cualquier $\delta<\alpha$ existe $k \in \omega$ tal que $\alpha_{k} \in E^{\prime}$ y $\delta \leq \alpha_{k}<\alpha$. De aquí resulta que el conjunto $A_{\alpha}^{k}$ es cofinal en $\alpha$. Por lo tanto, existe una sucesión $s_{k}^{\alpha} \subset A_{\alpha}^{k} \cap E \cap\left(\beta_{k}, \alpha\right]$ convergente a $\alpha$. Como $A_{\alpha}^{k} \cap E=E^{\prime} \cap \alpha$ tenemos que $s_{k}^{\alpha} \subset E^{\prime}$. Si $U \in \mathcal{U}_{n}$ es tal que $\left(\beta_{n}, \alpha\right] \subset U$ entonces $s_{k}^{\alpha} \cap\left(\beta_{n}, \alpha\right] \neq \emptyset$. Por lo tanto cualquier $\gamma \in s_{k}^{\alpha} \cap\left(\beta_{n}, \alpha\right] \subset E$ casi pertenece a $U$, lo cual es una contradicción.

Sean $\beta \in E$ y $\left\{U_{n}\right\}_{n \in \omega}$ como en la afirmación. Consideremos la familia centrada de cerrados $\left\{F_{n}\right\}_{n \in \omega}$, donde $F_{i}=U_{i} \cap\left\{\gamma_{n}(\beta): n \geq i\right\}$. Luego $F_{i} \neq \emptyset, F_{i} \subset U_{i} \in \mathcal{U}_{i}$ para todo $i \in \omega$ y $\bigcap_{i \in \omega} F_{i}=\emptyset$, lo cual es contradictorio con la supuesta completitud de $\left\{\mathcal{U}_{n}\right\}_{n \in \omega}$. Por lo tanto el espacio $X$ no es Čech completo.

En virtud del lema 2.24, el espacio $X^{n}$ es la unión de dos subespacios $A_{0}=\bigoplus_{\delta \in G} M_{\delta}$ y $A_{1}=\bigoplus_{\delta \notin G} M_{\delta}$, para todo $n \in \mathbb{N}$

Puesto que $X$ es regular y cada $M_{\delta}$ es segundo numerable, tenemos que cada subespacio $A_{j}$ es metrizable. También se cumple que cada subespacio $M_{\delta}$ es localmente Cech completo. En efecto, para cada punto de $M_{\delta}$ existe una vecindad $V$ primero numerable que contiene a lo más un punto no aislado. Del teorema 1.18 se desprende que el subespacio $V$ es Čech completo. Como consecuencia del teorema de Pasynkov (1.36), obtenemos que $M_{\delta}$ es Čech completo para toda $\delta<\omega_{1}$. El teorema 1.20 constata la Čech completitud de $A_{0}$ y $A_{1}$.

En el próximo teorema mostraremos que para los grupos topológicos, a diferencia de los espacios topológicos, la Čech completitud es finitamente aditiva (véase el ejemplo 2.7). Antes necesitaremos el siguiente resultado.

2.28. Lema. Sea $X$ un grupo topológico. Si $X$ es localmente Čech completo entonces $X$ es un espacio Čech completo.

Demostración. Consideremos el elemento identidad $e \in X$. Sea $U$ una vecindad Čech completa de $e$. Debido a la proposición 1.25, existe un subespacio compacto $K$ de carácter numerable que contiene a $e$. Sea $\left\{U_{i}\right\}_{i \in \omega} \subset \mathcal{T}(X)$ una base para $K$. Tomemos $W_{0}=W_{0}^{-1}, V_{0} \in \mathcal{T}(e, X)$ para los cuales $V_{0}^{2} \subset W_{0} \subset U_{0}$. Para 
cada $i \geq 1$ escojamos $W_{i}=W_{i}^{-1}, V_{i} \in \mathcal{T}(e, X)$ tales que $V_{i}^{2} \subset W_{i} \subset V_{i-1} \cap U_{i}$. De esta forma, obtenemos una familia $\left\{W_{i}\right\}_{i \in \omega} \subset \mathcal{T}(e, X)$ tal que $W_{i+1}^{2} \subset W_{i}=$ $W_{i}^{-1} \subset U_{i}$ para todo $i \in \omega$. De la contención $W_{i+1}^{2} \subset W_{i}$, se desprende que $\overline{W_{i+1}} \subset W_{i}$. En efecto, sea $x \in \overline{W_{i+1}}$. Como $x W_{i+1} \in \mathcal{T}(x, X)$, se sigue que $x W_{i+1} \cap W_{i+1} \neq \emptyset$. Luego, existen $y, w \in W_{i+1}$ tales que $y=x w$, es decir, $x=y w^{-1} \in W_{i+1} W_{i+1}^{-1}=W_{i+1}^{2} \subset W_{i}$. De aquí obtenemos que $\bigcap_{i \in \omega} W_{i}=\bigcap_{i \in \omega} \overline{W_{i}}$. El conjunto compacto $H=\bigcap_{i \in \omega} W_{i} \subset K$ es un subgrupo. Consideremos la función canónica $f: X \rightarrow X / H$ dada por $f(x)=H x$. Como $H$ es compacto se sigue que $f$ es cerrada y también una función perfecta, pues $f^{-1}(H x)=H x$ es un subespacio compacto para toda $x \in X$.

Notemos que $\chi(H, X) \leq \omega$, ya que $\chi(H, K) \leq \omega$ y $\chi(K, X) \leq \omega$. Como consecuencia de lo anterior y por ser $f$ una función abierta, obtenemos que $\chi(\{H\}, X / H) \leq$ $\chi(H, X) \leq \omega$. Dado que $H$ es cerrado, el espacio $X / H$ es homogéneo, y así que $\chi(X / H) \leq \omega$. Luego, $X / H$ es metrizable [Po], por lo tanto es un espacio paracompacto. Empleando el hecho de que la imagen inversa perfecta de un espacio paracompacto es paracompacta, obtenemos que $X$ es paracompacto, pues $X=f^{-1}(X / H)$. De la hipótesis sabemos que $X$ también es localmente Čech completo, de modo que del teorema de Pasynkov (1.36), se desprende que $X$ es un espacio Čech completo.

2.29. Teorema. Sea $X$ un grupo topológico. Si $X=A_{1} \cup A_{2} \cup \ldots \cup A_{n}$ donde $A_{i}$ es localmente Čech completo para todo $i \in\{1,2, \ldots, n\}$, entonces $X$ es Čech completo.

Demostración. Del teorema 2.12 sabemos que existe en $X$ un subespacio abierto, no vacío y Čech completo. Por ser $X$ un espacio homogéneo, concluimos que $X$ es localmente Čech completo. Por el lema anterior, el espacio $X$ es Čech completo.

Se dice que un grupo topológico $X$ es completo en el sentido de Raikov si para todo grupo topológico $H$ tal que $X$ es un subgrupo de $H$, denotado por $X<H$, se cumple que $X$ es un subespacio cerrado de $H$.

2.30. Proposición. Si un grupo topológico $X$ tiene un subespacio denso y Čech completo entonces $X$ es completo en el sentido de Raikov.

Demostración. Sea $H$ un grupo topológico tal que $X<H$. Como $\bar{X}<H$ se puede considerar que $H=\bar{X}$. Tenemos que demostrar que $X=H$. Si $H-X \neq \emptyset$, tomemos un $g \in H-X$. Sabemos que $g X \cap X=\emptyset$. Si $G$ es un denso Čech completo en $X$, entonces $G \cap g G \subset X \cap g X=\emptyset$. Los subespacios $G$ y $g G$ son densos en $H$ y por consiguiente, tanto ellos como su intersección, son $G_{\delta}$ en $H$. Resulta que una intersección numerable de abiertos densos en $H$ es vacía, lo cual contradice la propiedad de Baire de $H$ (véase 1.27 y 1.28).

De este resultado y de [Ch, teorema 1] obtenemos 
2.31. Corolario. Si un grupo topológico $X$ tiene un subespacio denso y Čech completo entonces $X$ es Čech completo.

Como consecuencia inmediata de este resultado, obtenemos que la Čech completitud se preserva bajo funciones abiertas en los grupos topológicos.

2.32. Lema. Sean $X, Y$ grupos topológicos. Si $f: X \rightarrow Y$ es una función abierta (que no necesariamente es un homomorfismo) y $X$ es Cech completo, entonces $Y$ es un espacio Čech completo.

Demostración. Como $X$ es casi Čech completo y $f$ es abierta, el teorema 1.33 nos asegura que el espacio $Y$ es casi Čech completo. Luego, existe un subespacio Čech completo $D \subset Y$ tal que $\bar{D}=Y$. Del corolario 2.31 se sigue que $Y$ es un espacio Čech completo. 


\section{Capítulo 3}

\section{Ultracompletitud}

En $[\mathrm{TkV}]$ se demostró que muchas propiedades no aditivas manifiestan aditividad en potencias finitas. Se probó, en particular, que el peso, el carácter, el pseudocarácter y la estrechez son aditivas en potencias finitas. En el ejemplo 2.26 se constata que existen modelos de ZFC donde la Čech completitud no es aditiva en potencia 2. De modo que es un planteamiento natural preguntar qué propiedades de tipo completitud son aditivas en potencias finitas. La respuesta es afirmativa para la propiedad de tipo puntual numerable (corolario 2.5); en este capítulo lo probamos para la ultracompletitud en espacios metrizables (teorema 3.6).

Debido a que la ultracompletitud es una propiedad más fuerte que la Čech completitud y es consecuencia de la compacidad local, muchas propiedades categóricas de la completitud son parecidas a las propiedades de la Čech completitud. Para el caso de la potencia $X^{\omega}$, establecemos que la ultracompletitud es numerablemente aditiva en $X^{\omega}$ (teorema 3.7), es decir, si $X^{\omega}=\bigcup_{i \in \omega} X_{i}$ y cada $X_{i}$ es ultracompleto, entonces $X^{\omega}$ es ultracompleto.

Bajo CH damos una respuesta positiva a una pregunta de Buhagiar y Yoshioka [BY2], probando que existen espacios Čech completos numerablemente compactos que no son ultracompletos (ejemplo 3.14). En lo referente a los grupos topológicos, demostramos que la ultracompletitud y la compacidad local son equivalentes (teorema 3.10$)$.

\section{Aditividad en potencias.}

En esta sección probaremos que la ultracompletitud, a diferencia de la Čech completitud, es aditiva en potencias finitas. También estableceremos que la ultracompletitud es numerablemente aditiva en $X^{\omega}$. En el caso de los grupos topológicos obtenemos que la compacidad local es equivalente a la ultracompletitud.

3.1. Teorema. Sea $X=A_{1} \cup A_{2} \cup \ldots \cup A_{n}$ donde $A_{i}$ es un subespacio ultracompleto para toda $i=1,2, \ldots, n$. Si $X=\overline{A_{i}}$ para toda $i \in\{1,2, \ldots, n\}$ entonces $X$ es ultracompleto. 
Demostración. Como $X=\overline{A_{i}}$, obtenemos que ${\overline{A_{i}}}^{\beta X}=\beta X$. Para cada $i \in$ $\{1,2, \ldots, n\}$, escojamos una base numerable $\mathcal{U}_{i} \subset \mathcal{T}(\beta X)$ para $A_{i}$. Fijémonos en la familia

$$
\mathcal{W}=\left\{U_{1} \cup U_{2} \cup \ldots \cup U_{n}: U_{i} \in \mathcal{U}_{i} \text { para cada } i=1,2, \ldots, n\right\} .
$$

Es claro que $\mathcal{W}$ es numerable. Probemos que $\mathcal{W} \subset \mathcal{T}(\beta X)$ es base para $X$ en $\beta X$. Si $V \in \mathcal{T}(X, \beta X)$ entonces $A_{i} \subset V$ para toda $i \leq n$. De aquí se sigue que para cada $i \in\{1,2, \ldots, n\}$ existe $U_{j(i)} \in \mathcal{U}_{i}$ para el cual $A_{i} \subset U_{j(i)} \subset V$. Por lo tanto $\bigcup_{i=1}^{n} U_{j(i)} \in \mathcal{W} \mathrm{y}$

$$
X=\bigcup_{i=1}^{n} A_{i} \subset \bigcup_{i=1}^{n} U_{j(i)} \subset V
$$

Para el caso en que alguno de los subespacios $A_{i}$ no sea denso, tenemos el siguiente resultado.

3.2. Corolario. Supongamos que $X=A_{1} \cup A_{2} \cup \ldots \cup A_{n}$ donde $A_{i}$ es un subespacio ultracompleto para toda $i=1,2, \ldots, n$. Entonces existe $G \in \mathcal{T}^{*}(X)$ tal que $\bar{G}$ es ultracompleto.

Demostración. Procederemos por inducción sobre $n$. Como el caso $n=1$ es evidente, supongamos que $n \geq 2$ y se demostró nuestro teorema para cada $k<n$. Si $X=\overline{A_{i}}$ para toda $i \leq n$, el teorema 3.1 muestra que $X$ es ultracompleto y por lo tanto sirve $G=X$. Supongamos que existe $i_{0} \in\{1,2, \ldots, n\}$ para el cual $X \neq \overline{A_{i_{0}}}$. Podemos suponer sin pérdida de generalidad, que $i_{0}=1$. Dado que $X-\overline{A_{1}} \subset A_{2} \cup A_{3} \cup \cdots \cup A_{n}$ existe $W \in \mathcal{T}^{*}(X)$ para el cual $W \subset \bar{W} \subset X-\overline{A_{1}} \subset$ $A_{2} \cup A_{3} \cup \cdots \cup A_{n}$. Esto nos lleva a que $\bar{W}=A_{2}^{\prime} \cup A_{3}^{\prime} \cup \cdots \cup A_{n}^{\prime}$ donde $A_{j}^{\prime}=A_{j} \cap \bar{W}$ es ultracompleto para $2 \leq j \leq n$. Por hipótesis de inducción existe $H=\bar{W} \cap U \neq \emptyset$ para algún $U \in \mathcal{T}^{*}(X)$ tal que $\bar{H}$ es ultracompleto. Dado que $W \cap U \in \mathcal{T}^{*}(X)$, existe $G \in \mathcal{T}^{*}(X)$ para el cual $G \subset \bar{G} \subset W \cap U \subset \bar{H}$. Como $\bar{H}$ es ultracompleto, el subespacio $\bar{G}$ también lo es.

Se dice que un conjunto $A \subset X$ es acotado en $X$ si para toda función continua $f: X \rightarrow \mathbb{R}$, el conjunto $f(A) \subset \mathbb{R}$ es acotado. Los siguientes tres resultados aparecen en $[\mathrm{PT}]$.

3.3. Lema. Un conjunto $A \subset X$ es acotado en $X$ si y sólo si para cualquier familia discreta $\mathcal{B} \subset \mathcal{T}^{*}(X)$ el conjunto $\mathcal{B}_{A}=\{B \in \mathcal{B}: B \cap A \neq \emptyset\}$ es finito.

Demostración. Sea el conjunto $A \subset X$ acotado en $X$. Consideremos una familia discreta $\mathcal{B} \subset \mathcal{T}^{*}(X)$ y supongamos que $\mathcal{B}_{A}$ es infinito. Tomemos $\left\{U_{n}\right\}_{n \in \mathbb{N}} \subset \mathcal{B}_{A}$ y un $y_{n} \in U_{n} \cap A$ para todo $n \in \mathbb{N}$. Fijémonos en la función continua $f:\left\{y_{n}\right\}_{n \in \mathbb{N}} \rightarrow$ $\mathbb{R}$ dada por $f\left(y_{n}\right)=n$. Para cada $n \in \mathbb{N}$ existe una función continua $f_{n}: X \rightarrow[0,1]$ 
tal que $f_{n}\left(y_{n}\right)=1$ y $f_{n}(x)=0$ para todo $x \in X-U_{n}$. Consideremos la función $\widehat{f}: X \rightarrow \mathbb{R}$ con $\widehat{f}(x)=\Sigma_{n \in \mathbb{N}} n f_{n}(x)$. Claramente $\widehat{f}\left(y_{n}\right)=f\left(y_{n}\right)$ para todo $n$ y por lo tanto $\widehat{f}$ es no acotada. Probemos que $\widehat{f}$ es continua. Tomemos $x \in X$. Por ser $\mathcal{B}$ una familia discreta, existe $U_{x} \in \mathcal{T}(x, X)$ tal que $\left|\left\{U_{x} \cap U_{n} \neq \emptyset: U_{n} \in \mathcal{B}_{A}\right\}\right| \leq 1$. Si $U_{x} \cap U_{n}=\emptyset$ para todo $n \in \omega$ entonces la función $f\left\lceil U_{x}=f_{1}\left\lceil U_{1}\right.\right.$ es continua. Ahora bien, si $U_{x} \cap U_{m} \neq \emptyset$ para un único $m$ entonces $f\left\lceil U_{x}=f_{m}\left\lceil U_{m}\right.\right.$ es una función continua. De aquí resulta que $\widehat{f}$ es una función continua en cada elemento de la cubierta abierta $\left\{U_{x}\right\}_{x \in X}$ de $X$, y por lo tanto $\widehat{f}$ es una función continua en $X$, lo que es contradictorio con el hecho de ser el conjunto $A$ acotado en $X$.

Para probar la suficiencia, supongamos que $A \subset X$ no es acotado en $X$. Luego, existe una función continua $f: X \rightarrow \mathbb{R}$ tal que $f(A) \subset \mathbb{R}$ no es acotado. Consideremos el conjunto no acotado $Y=\left\{y_{n}: y_{n}=f\left(a_{n}\right)\right.$ con $\left.a_{n} \in A\right\} \subset \mathbb{R}$. Sea $x_{1} \in Y$. Para cada $n \in \mathbb{N}$ tomemos $x_{n+1} \in Y$ tal que $\left|x_{n+1}\right|>\left|x_{n}\right|+1$. El conjunto $\left\{x_{n}\right\}_{n \in \mathbb{N}} \subset \mathbb{R}$ es discreto y cerrado. Por lo tanto existe una familia discreta $\left\{V_{n}\right\}_{n \in \mathbb{N}} \subset \mathcal{T}(\mathbb{R})$ para la cual $x_{n} \in V_{n}$. De aquí concluimos que $\mathcal{B}=\left\{U_{n}\right\}_{n \in \mathbb{N}} \subset \mathcal{T}(X)$ donde $U_{n}=f^{-1}\left(V_{n}\right)$, es una familia discreta tal que $\left|\mathcal{B}_{A}\right|=\omega$, de donde $A$ es un conjunto acotado.

3.4. Teorema. Si $X$ es un espacio ultracompleto, entonces el conjunto $Y=\{x \in$ $X: X$ no es localmente compacto en $x\}$ es acotado en $X$.

Demostración. Supongamos que $Y$ no es acotado en $X$. Por el lema, existe una familia discreta numerable $\mathcal{B}=\left\{U_{n}\right\}_{n \in \omega}$ tal que $U_{n} \cap Y \neq \emptyset$ para todo $n \in \omega$. Consideremos la familia $\left\{V_{n}\right\}_{n \in \omega} \subset \mathcal{T}(\beta X)$, tal que $U_{n}=X \cap V_{n}$ para todo $n \in \omega$. Sea $\left\{B_{n}\right\}_{n \in \omega} \subset \mathcal{T}(\beta X)$ una base para $X$. Puesto que $X$ no es localmente compacto para cualquier $y \in Y$, tenemos que $V_{n} \cap(\beta X-X) \neq \emptyset$ para todo $n \in \omega$. Pues de lo contrario, se cumple que $U_{n}=V_{n} \subset X$ para algún $n$. Luego, $y_{n} \in W \subset \bar{W} \subset U_{n} \subset$ $X$ donde $W \in \mathcal{T}(\beta X)$, y por lo tanto $X$ es localmente compacto en $y_{n} \in Y$.

Tomemos la sucesión $Z=\left\{z_{n}\right\}_{n \in \omega}$ donde $z_{n} \in B_{n} \cap V_{n} \cap(\beta X-X)$. Como $Z \cap B_{n} \neq \emptyset$ para todo $n \in \omega$, tenemos que $\bar{Z} \cap X \neq \emptyset$. Sea $x \in \bar{Z} \cap X$. Por ser $\mathcal{B}$ una familia discreta, existe $U \in \mathcal{T}(x, X)$ tal que la intersección de $U$ con los elementos de $\mathcal{B}$ es diferente del vacío, en a lo más un elemento de $\mathcal{B}$. Sea $U=X \cap U_{x}$ donde $U_{x} \in \mathcal{T}(\beta X)$. De la igualdad $U \cap U_{n}=X \cap V_{n} \cap U_{x}$, se desprende que $U_{x} \cap V_{n}=\emptyset$ para casi todo $n \in \mathbb{N}$, lo cual contradice que $x \in \bar{Z}$.

3.5. Teorema. Sea $X$ un espacio metrizable. Luego, $X$ es ultracompleto si y sólo si el conjunto $Y=\{x \in X: X$ no es localmente compacto en $x\}$ es compacto.

Demostración. Supongamos que $X$ es ultracompleto. Dado que $X$ es paracompacto y $Y$ es cerrado en $X$, se desprende que $Y$ es paracompacto. Por otro lado, del teorema 3.4 sabemos que el conjunto $Y$ es acotado en $X$ y por ser $X$ un espacio normal, obtenemos que $Y$ es pseudocompacto, de donde el conjunto $Y$ es compacto. 
Para probar la suficiencia, observemos que $X-Y$ es abierto en $\beta X$ e $Y$ es un subconjunto $G_{\delta}$ de $\beta X$. En efecto, tomemos $z \in X-Y$. Por ser $X-Y$ abierto en $X$, existe $U \in \mathcal{T}(z, X)$ tal que $z \in U \subset \bar{U}^{X} \subset X-Y$ donde $\bar{U}^{X}$ es compacto. Sea $V \in \mathcal{T}(\beta X)$ para el cual $U=X \cap V$. Como $\bar{U}^{X}$ es compacto y denso en $\bar{U}$ tenemos que $\bar{U}^{X}=\bar{U}$. De la igualdad $\bar{U}=\bar{V}$, se sigue que $z \in V \subset X-Y$, de donde $X-Y$ es abierto en $\beta X$. Para probar que el compacto $Y$ es un subconjunto $G_{\delta}$ de $\beta X$, basta verificar que $\chi(Y, \beta X) \leq \omega$. Consideremos una base numerable $\mathcal{B}$ en $Y$. Observemos que para todo $U \in \mathcal{B}$ existe $U_{n} \in \mathcal{T}(X)$ tal que $U=Y \cap U_{n}$ donde $\operatorname{diam} U_{n}<\operatorname{diam} U+\frac{1}{n}$. Verifiquemos que la familia $\mathcal{B}^{\prime}=\left\{U_{n}: U \in \mathcal{B}\right.$ y $\left.n \in \mathbb{N}\right\} \subset \mathcal{T}(X)$ es base exterior para $Y$. Tomemos $y \in Y$ y $V \in \mathcal{T}(y, X)$. Existe $r>0$ tal que $B(y, r) \subset V$. Escojamos $U \in \mathcal{B}$ para el cual $y \in U \subset B\left(y, \frac{r}{4}\right) \cap Y$. Si $n \in \mathbb{N}$ satisface $\frac{1}{n}<\frac{r}{2}$ entonces $y \in U_{n} \subset B(y, r) \subset V$, de donde $\mathcal{B}^{\prime}$ es base exterior para $Y$. Luego, la familia $\gamma=\left\{\bigcup \lambda: \lambda \subset \mathcal{B}^{\prime}, Y \subset \bigcup \lambda\right.$, y $\left.|\lambda|<\omega\right\} \subset \mathcal{T}(X)$ es una base numerable para $Y$ en $X$. Es claro que $|\gamma| \leq \omega$. Sea $W \in \mathcal{T}(X)$ con $Y \subset W$. Como $\mathcal{B}^{\prime}$ es base exterior para $Y$, tenemos que para cada $y \in Y$ existe $U_{y} \in \mathcal{B}^{\prime}$ para el cual $y \in U_{y} \subset W$. Por ser $Y$ compacto, existen $y_{1}, y_{2}, \ldots, y_{k} \in Y$ con $Y \subset \bigcup_{i=1}^{k} U_{y_{i}} \subset W$. Dado que $\bigcup_{i=1}^{k} U_{y_{i}} \in \gamma$, se sigue que $\gamma$ es base para $Y$. Sea $\gamma=\left\{U_{i}\right\}_{i \in \mathbb{N}}$, luego $Y=\bigcap_{i \in \mathbb{N}} U_{i}$. Consideremos los conjuntos $V_{i} \in \mathcal{T}(\beta X)$ tales que $U_{i}=X \cap V_{i}$. La familia $\mathcal{V}=\left\{V_{i}\right\}_{i \in \mathbb{N}} \subset \mathcal{T}(\beta X)$ es base para $Y$. En efecto, sea $V \in \mathcal{T}(Y, \beta X)$. Por ser $Y$ compacto, existe $U \in \mathcal{T}(\beta X)$ para el cual $Y \subset U \subset \bar{U} \subset V$. Luego $Y \subset X \cap U$, así que, existe $V_{i_{0}} \in \mathcal{V}$ para algún $i_{0} \in \mathbb{N}$ con $Y \subset X \cap V_{i_{0}} \subset X \cap U$. De aquí resulta que $Y \subset V_{i_{0}} \subset \overline{V_{i_{0}}}=\overline{X \cap V_{i_{0}}} \subset \overline{X \cap U}=\bar{U} \subset V$, es decir, $\mathcal{V}$ es base para $Y$, y por lo tanto $\chi(Y, \beta X) \leq \omega$. Finalmente, para concluir nuestra demostración, probemos que la familia $\left\{V_{i} \cup(X-Y): V_{i} \in \mathcal{V}, i \in \mathbb{N}\right\} \subset \mathcal{T}(\beta X)$ es base para $X$ en $\beta X$. Sea $V \in \mathcal{T}(X, \beta X)$. Como $Y \subset V$, existe $V_{i} \in \mathcal{V}$ para el cual $Y \subset V_{i} \subset V$ y en consecuencia $X=(X-Y) \cup Y \subset(X-Y) \cup V_{i} \subset(X-Y) \cup V \subset V$.

3.6. Teorema. Sea $X$ un espacio metrizable. Supongamos que $X^{n}=X_{1} \cup X_{2} \cup$ $\ldots \cup X_{n}$ donde cada $X_{i}$ es ultracompleto. Entonces $X$ es ultracompleto.

Demostración. Aplicando el teorema 3.5, para cada $i \leq n$ existe un compacto $K_{i} \subset X_{i}$ tal que $X_{i}-K_{i}$ es localmente compacto. Denotemos por $p_{j}: X^{n} \rightarrow X$ la proyección de $X^{n}$ sobre su $j$-ésimo factor y consideremos $K=\bigcup_{i, j \leq n} p_{j}\left(K_{i}\right)$. El conjunto $K \subset X$ es compacto y además $Y^{n} \cap\left(K_{1} \cup \ldots \cup K_{n}\right)=\emptyset$, donde $Y=X-K$. De aquí resulta que $Y_{i}=X_{i} \cap Y^{n}$ es un subconjunto abierto del espacio localmente compacto $X_{i}-K_{i}$ para todo $i \leq n$. De modo que $Y^{n}=Y_{1} \cup Y_{2} \cup \ldots \cup Y_{n}$ donde cada $Y_{i}$ es localmente compacto. El Corolario 1.4 de $[\mathrm{TkV}]$, nos asegura que $Y$ es localmente compacto. De aquí concluimos que el conjunto de puntos donde el espacio metrizable $X$ no es localmente compacto, es un conjunto compacto. Por lo tanto, del teorema 3.5 obtenemos que $X$ es ultracompleto. 
3.7. Teorema. Si $X^{\omega}$ es unión numerable de subespacios ultracompletos, entonces el espacio $X^{\omega}$ es ultracompleto.

Demostración. Como $X^{\omega}=\left(X^{\omega}\right)^{\omega}=\bigcup_{i \in \omega} X_{i}$, tenemos que los Lemas 1 y 2 de [TkM] garantizan la existencia de $i_{0} \in \omega$ y un subespacio cerrado $Y$ de $X_{i_{0}}$, tal que existe una función abierta de $Y$ sobre $X^{\omega}$. De 1.56 y 1.57 se sigue que $X^{\omega}$ es ultracompleto.

3.8. Ejemplo. Existe un espacio segundo numerable no ultracompleto que es unión de dos subespacios ultracompletos.

Demostración. Sea $H$ el erizo de Kowalsky con un número numerable de espinas. El único punto de no compacidad local de $H$ es el vértice $h$. Por lo tanto, el teorema 3.5 nos garantiza que $H$ es ultracompleto.

Para cada $i \in \omega$, tomemos una copia homeomorfa $H_{i}$ de $H$, con $h_{i}$ la correspondiente copia de $h$ en $H_{i}$. El espacio $X=\bigoplus_{i \in \omega} H_{i}$ es separable y metrizable. El conjunto $\left\{h_{i}: i \in \omega\right\}$ de todos los puntos de no compacidad local de $X$ no es compacto. Aplicando nuevamente el teorema 3.5, vemos que el espacio $X$ no es ultracompleto. Sin embargo, tenemos que $X=A \cup B$ donde $A=\left\{h_{i}: i \in \omega\right\}$ y $B=X-A$ son localmente compactos y por consiguiente ultracompletos.

El siguiente teorema muestra que la ultracompletitud y la compacidad local son propiedades equivalentes para el caso de los grupos topológicos; para su demostración haremos uso del siguiente lema que ha sido probado en 2.28.

3.9. Lema. Sea $G$ un grupo topológico. Si existe un subconjunto compacto $K \subset G$ tal que $\chi(K, G) \leq \omega$, entonces $G$ es paracompacto.

3.10. Teorema. Sea $G$ un grupo topológico. Si existe un subconjunto abierto y no vacío $U \subset G$ tal que $\bar{U}$ es ultracompleto, entonces $G$ es localmente compacto. En particular, cada grupo ultracompleto es localmente compacto.

Demostración. Sea $\mathcal{C}=\{x \in \bar{U}: \bar{U}$ es localmente compacto en $x\}$. Como el conjunto de puntos de compacidad local es abierto en cualquier espacio, tenemos que $\mathcal{C}$ es abierto en $\bar{U}$. Supongamos que $\mathcal{C} \neq \emptyset$. Luego $\mathcal{C} \cap U \neq \emptyset$. Tomemos un $x \in \mathcal{C} \cap U$. Existe $V \in \mathcal{T}(x, \bar{U})$ tal que $\bar{V}$ es compacto. Dado que $V \cap U \in \mathcal{T}(x, G)$, elijamos un $W \in \mathcal{T}(x, G)$ para el cual $W \subset \bar{W} \subset V \cap U$. De la contención $\bar{W} \subset \bar{V}$, se desprende que $\bar{W}$ es compacto, y por lo tanto $G$ es localmente compacto en $x$. Por ser $G$ un espacio homogéneo, se sigue que $G$ es localmente compacto.

Ahora consideremos el caso en que $\mathcal{C}=\emptyset$. Aplicando el teorema 3.4, obtenemos que $\bar{U}$ es acotado en $\bar{U}$, es decir, $\bar{U}$ es pseudocompacto. Puesto que $U$ es Čech completo, pues $U \in \mathcal{T}(\bar{U})$ (véase teorema 1.19), tenemos que existe un 
compacto $K \subset G$ tal que $\chi(K, G) \leq \omega$ (teorema 1.25). Del lema 3.9 se deduce que $G$ es paracompacto. De aquí resulta que el cerrado $\bar{U}$ es paracompacto y pseudocompacto. Por lo tanto $\bar{U}$ es compacto, lo cual es contradictorio con la suposición de que $\mathcal{C}=\emptyset$.

3.11. Corolario. Sea $G$ un grupo topológico. Si existe un subespacio ultracompleto $U \in \mathcal{T}^{*}(G)$, entonces $G$ es localmente compacto.

3.12. Corolario. Si un grupo topológico $G$ es una unión finita de subespacios ultracompletos, entonces $G$ es localmente compacto.

Demostración. El corolario 3.2 garantiza la existencia de un abierto y no vacío $U \subset G$ tal que $\bar{U}$ es ultracompleto. Aplicando el teorema 3.10 obtenemos que $G$ es localmente compacto.

\section{Un ejemplo de un espacio numerablemente compacto, Čech completo y no ultracompleto.}

Suponiendo $\mathrm{CH}$, construiremos un subespacio de $\beta \omega$ que es numerablemente compacto, Čech completo y no ultracompleto, respondiendo de esta forma, a una pregunta planteada en [BY2]. Aquí $\beta \omega$ es la compactificación de Stone-Čech de $\omega$.

Recordemos que $x \in X$ es un punto $P$ de $X$ si todo conjunto $G_{\delta}$ que contiene a $x$ es una vecindad de $x$. Por el teorema 1.46 sabemos que, suponiendo $\mathrm{CH}$, existen puntos $P$ en $\omega^{*}=\beta \omega-\omega$. En lo que sigue, denotaremos por $\mathcal{T}_{c}^{*}(X)$ el conjunto de todos los subconjuntos no vacíos y abierto-cerrados de $X$.

3.13. Lema. Supóngase que es válida la Hipótesis del Continuo. Sea $p \in \omega^{*}$ un punto $P$. Existen familias $\left\{U_{\alpha}: \alpha<\omega_{1}\right\},\left\{V_{\alpha}: \alpha<\omega_{1}\right\} \subset \mathcal{T}_{c}^{*}\left(\omega^{*}\right)$ que satisfacen

(1) $U_{\beta} \subset U_{\beta^{\prime}} V_{\beta} \subset V_{\beta^{\prime}}$ si $\beta<\beta^{\prime}<\omega_{1}$;

(2) $\left(\bigcup_{\beta<\omega_{1}} U_{\beta}\right) \cap\left(\bigcup_{\beta<\omega_{1}} V_{\beta}\right)=\emptyset$;

(3) $\overline{\left(\bigcup_{\beta<\omega_{1}} U_{\beta}\right) \bigcup\left(\bigcup_{\beta<\omega_{1}} V_{\beta}\right)}=\omega^{*}$;

(4) $p \in \overline{\left(\bigcup_{\beta<\omega_{1}} U_{\beta}\right)} \cap \overline{\left(\bigcup_{\beta<\omega_{1}} V_{\beta}\right)}-\left(\left(\bigcup_{\beta<\omega_{1}} U_{\beta}\right) \bigcup\left(\bigcup_{\beta<\omega_{1}} V_{\beta}\right)\right)$.

Demostración. Sea $\mathcal{O}=\left\{O_{\alpha}: \alpha<\omega_{1}\right\} \subset \mathcal{T}_{c}^{*}\left(\omega^{*}\right)$ una $\pi$-base en $\omega^{*}$ con $p \notin O_{\alpha}$ para todo $\alpha<\omega_{1}$. Tomemos una base local $\left\{W_{\alpha}: \alpha<\omega_{1}\right\} \subset \mathcal{T}_{c}^{*}\left(\omega^{*}\right)$ en el punto $p$ para la cual, $W_{\alpha^{\prime}} \subset W_{\alpha}$ en cuanto $\alpha<\alpha^{\prime}$. Sean $x, y \in \omega^{*}-\{p\}$ con $x \neq y$. Consideremos $W_{0}^{\prime}, W_{0}^{\prime \prime} \in \mathcal{T}_{c}^{*}\left(\omega^{*}\right)$ tales que $W_{0}^{\prime} \subset W_{0}, W_{0}^{\prime \prime} \subset W_{0}, W_{0}^{\prime} \cap W_{0}^{\prime \prime}=\emptyset \mathrm{y}$ $\left(W_{0}^{\prime} \cup W_{0}^{\prime \prime}\right) \bigcap\{x, y, p\}=\emptyset$. Existen $U_{0}^{\prime}, V_{0}^{\prime} \in \mathcal{T}_{c}^{*}\left(\omega^{*}\right)$ para los cuales $x \in U_{0}^{\prime}, y \in V_{0}^{\prime}$, $p \notin\left(U_{0}^{\prime} \bigcup V_{0}^{\prime}\right), U_{0}^{\prime} \bigcap V_{0}^{\prime}=\emptyset \mathrm{y}\left(U_{0}^{\prime} \bigcup V_{0}^{\prime}\right) \bigcap\left(W_{0}^{\prime} \bigcup W_{0}^{\prime \prime}\right)=\emptyset$. Sean $U_{0}^{\prime \prime}=U_{0}^{\prime} \bigcup W_{0}^{\prime}$, 
$V_{0}^{\prime \prime}=V_{0}^{\prime} \bigcup W_{0}^{\prime \prime}$. Si $\left(U_{0}^{\prime \prime} \bigcup V_{0}^{\prime}\right) \bigcap O_{0} \neq \emptyset$ tomamos $U_{0}=U_{0}^{\prime \prime}, V_{0}=V_{0}^{\prime \prime}$. En caso contrario hagamos $U_{0}=U_{0}^{\prime \prime} \cup O_{0}$ y $V_{0}=V_{0}^{\prime \prime}$.

Sea $\alpha<\omega_{1}$. Supongamos que tenemos las familias $\left\{U_{\beta}: \beta<\alpha\right\} \subset \mathcal{T}_{c}^{*}\left(\omega^{*}\right)$, $\left\{V_{\beta}: \beta<\alpha\right\} \subset \mathcal{T}_{c}^{*}\left(\omega^{*}\right)$ que satisfacen

$\left(1_{\alpha}\right) \quad U_{\beta} \subset U_{\beta^{\prime}} \quad V_{\beta} \subset V_{\beta^{\prime}}$ si $\beta<\beta^{\prime}<\alpha$;

$\left(2_{\alpha}\right) \quad\left(\bigcup_{\beta<\alpha} U_{\beta}\right) \cap\left(\bigcup_{\beta<\alpha} V_{\beta}\right)=\emptyset$ у $p \notin\left(\bigcup_{\beta<\alpha} U_{\beta}\right) \bigcup\left(\bigcup_{\beta<\alpha} V_{\beta}\right)$;

$\left(3_{\alpha}\right) \quad\left(U_{\beta} \cup V_{\beta}\right) \bigcap O_{\beta} \neq \emptyset$ para todo $\beta<\alpha$;

$\left(4_{\alpha}\right) \quad U_{\beta} \bigcap W_{\beta} \neq \emptyset$ y $V_{\beta} \bigcap W_{\beta} \neq \emptyset$ para todo $\beta<\alpha$.

Sean $F_{\alpha}=\overline{\bigcup_{\beta<\alpha} U_{\beta}}$ y $G_{\alpha}=\overline{\bigcup_{\beta<\alpha} V_{\beta}}$. Por ser $\omega^{*}$ un espacio $F$ (teorema 1.52), tenemos que $F_{\alpha} \cap G_{\alpha}=\emptyset$. Dado que $p \notin\left(\bigcup_{\beta<\alpha} U_{\beta}\right) \cup\left(\bigcup_{\beta<\alpha} V_{\beta}\right)$ y como $p$ es un punto $P$, existe $W \in \mathcal{T}\left(p, \omega^{*}\right)$ con $W \subset\left(\omega^{*}-\bigcup_{\beta<\alpha} U_{\beta}\right) \cap\left(\omega^{*}-\bigcup_{\beta<\alpha} V_{\beta}\right)$. De aquí se desprende que

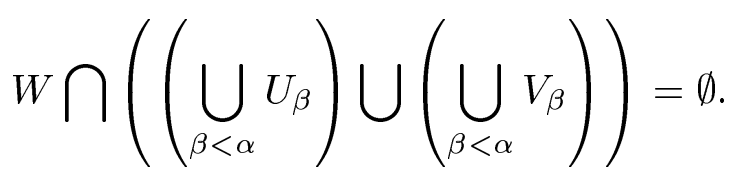

Por lo tanto $p \notin F_{\alpha} \cup G_{\alpha}$. Sea $\beta_{\alpha} \geq \alpha$ tal que $W_{\beta_{\alpha}} \subset W$.

Por normalidad de $\omega^{*}$, escojamos $U_{\alpha}^{\prime}, V_{\alpha}^{\prime} \subset \omega^{*}$ abiertos y ajenos para los cuales $F_{\alpha} \subset U_{\alpha}^{\prime}$ y $G_{\alpha} \subset V_{\alpha}^{\prime}$, con $p \notin\left(U_{\alpha}^{\prime} \cup V_{\alpha}^{\prime}\right)$. Dado que $F_{\alpha}$ y $G_{\alpha}$ son compactos y que $\omega^{*}$ tiene una base de abierto-cerrados, podemos suponer, sin pérdida de generalidad, que los abiertos $U_{\alpha}^{\prime}$ y $V_{\alpha}^{\prime}$ también son conjuntos cerrados. Sean $W^{\prime}, W^{\prime \prime} \subset W_{\beta_{\alpha}}$ ajenos, abierto-cerrados, para los cuales se satisface $\left(W^{\prime} \cup W^{\prime \prime}\right) \cap\left(U_{\alpha}^{\prime} \cup V_{\alpha}^{\prime}\right)=\emptyset$. Hagamos $U_{\alpha}^{\prime \prime}=U_{\alpha}^{\prime} \bigcup W^{\prime}, V_{\alpha}^{\prime \prime}=V_{\alpha}^{\prime} \bigcup W^{\prime \prime}$. Para terminar nuestra construcción, si sucede que $\left(U_{\alpha}^{\prime \prime} \cup V_{\alpha}^{\prime \prime}\right) \bigcap O_{\alpha} \neq \emptyset$ tomemos $U_{\alpha}=U_{\alpha}^{\prime \prime}$ y $V_{\alpha}=V_{\alpha}^{\prime \prime}$. En caso contrario, elijamos $U_{\alpha}=U_{\alpha}^{\prime \prime} \bigcup O_{\alpha}$ y $V_{\alpha}=V_{\alpha}^{\prime \prime}$. Por la construcción de $U_{\alpha}$ y $V_{\alpha}$, es fácil verificar que se satisfacen las propiedades $\left(1_{\alpha+1}\right)-\left(4_{\alpha+1}\right)$, y por lo tanto el proceso de inducción es válido hasta $\omega_{1}$. Como consecuencia de lo anterior, obtenemos las familias $\left\{U_{\alpha}: \alpha<\omega_{1}\right\}$ y $\left\{V_{\alpha}: \alpha<\omega_{1}\right\}$ que cumplen con $\left(1_{\alpha}\right)-\left(4_{\alpha}\right)$ para todo $\alpha<\omega_{1}$.

Para concluir nuestra demostración, verifiquemos que las familias $\left\{U_{\alpha}: \alpha<\omega_{1}\right\}$ y $\left\{V_{\alpha}: \alpha<\omega_{1}\right\}$ obtenidas de esta forma, satisfacen las condiciones del lema. Si $\beta<\beta^{\prime}<\omega_{1}$ entonces, de la condición $\left(1_{\alpha}\right)$ se desprende que $U_{\beta} \subset U_{\beta^{\prime}} \mathrm{y}$ $V_{\beta} \subset V_{\beta^{\prime}}$, y por lo tanto se cumple (1). La validez de (2) es consecuencia inmediata de la constatación de $\left(2_{\alpha}\right)$ para todo $\alpha<\omega_{1}$. Como $\left(3_{\alpha}\right)$ se satisface para todo $\alpha<\omega_{1}$, tenemos que (3) es verdadera. Para verificar la última condición, notemos que de $\left(2_{\alpha}\right)$ se sigue que $p \notin\left(\left(\bigcup_{\beta<\omega_{1}} U_{\beta}\right) \bigcup\left(\bigcup_{\beta<\omega_{1}} V_{\beta}\right)\right)$. Ahora tomemos un $U \in \mathcal{T}\left(p, \omega^{*}\right)$. Luego, $W_{\alpha} \subset U$ para algún $\alpha<\omega_{1}$. De la propiedad $\left(4_{\alpha+1}\right)$ se 
desprende que $U_{\alpha} \cap W_{\alpha} \neq \emptyset$. Por lo tanto $\left(\bigcup\left\{U_{\beta}: \beta<\omega_{1}\right\}\right) \cap U \neq \emptyset$, así que $p \in \overline{\bigcup\left\{U_{\beta}: \beta<\omega_{1}\right\}}$. De manera análoga, obtenemos que $p \in \overline{\bigcup\left\{V_{\beta}: \beta<\omega_{1}\right\}}$ y por consiguiente, se satisface la condición (4).

3.14. Ejemplo. La Hipótesis del Continuo implica que existe un espacio Čech completo, numerablemente compacto y no ultracompleto.

Demostración. Sea $A \subset \omega^{*}$ un conjunto discreto y numerable. Luego, el conjunto $\bar{A}-A$ es homeomorfo a $\omega^{*}$, así que podemos aplicar el lema 3.16 para encontrar un punto $P$ en el subespacio $\bar{A}-A$ y familias $\left\{U_{\alpha}: \alpha<\omega_{1}\right\},\left\{V_{\alpha}: \alpha<\omega_{1}\right\}$ de subconjuntos abierto-cerrados en $\bar{A}-A$ que verifican las condiciones (1),(2),(3) y (4) del mencionado lema.

Tomemos $X=\omega^{*}-(F \cup A)$ donde $F=(\bar{A}-A)-\bigcup_{\alpha<\omega_{1}} V_{\alpha}$. Es evidente que $X$ es Čech completo. Afirmamos que $X$ es numerablemente compacto y no es ultracompleto.

Sea $\left\{W_{\alpha}: \alpha<\omega_{1}\right\} \subset \mathcal{T}_{c}^{*}(\bar{A}-A)$ una base local en $p$ para la cual, si $\alpha<\alpha^{\prime}$ entonces $W_{\alpha^{\prime}} \subset W_{\alpha}$.

Supongamos que $X$ es ultracompleto. Dado que $\omega^{*}$ es una compactificación de $X$ y $\omega^{*}-X=F \cup A$, existe una sucesión de subespacios compactos $\left\{K_{n}: n \in \omega\right\}$ en $F \cup A$ con $K_{n} \subset K_{n+1}$, que constata la ultracompletitud del espacio $X$, es decir, para cualquier compacto $K \subset F \cup A$ existe un $n_{0}$ tal que $K \subset K_{n_{0}}$. Para cada $n \in \omega$, tomemos $A_{n}=A \cap K_{n}$. Como $\overline{A_{n}} \subset A \cup F$, se tiene que $A_{n}^{*}=\overline{A_{n}}-A_{n} \subset F$. Del teorema 1.38 (iv), cada conjunto $A_{n}^{*}$ es abierto-cerrado en $\bar{A}-A$. Afirmamos que $p$ no pertenece a $A_{n}^{*}$ para todo $n \in \omega$. En efecto, supongamos que $p \in A_{n_{0}}^{*}$ para algún $n_{0} \in \omega$. Dado que $A_{n_{0}}^{*}$ es abierto en $\bar{A}-A$, de la condición (4) del lema 3.13 , obtenemos que $A_{n_{0}}^{*} \cap\left(\bigcup_{\alpha<\omega_{1}} V_{\alpha}\right) \neq \emptyset$, lo cual contradice que $A_{n_{0}}^{*} \subset F$. De lo anterior se desprende que dado $n \in \omega$, existe un $\alpha_{n}<\omega_{1}$ tal que $W_{\alpha_{n}} \cap A_{n}^{*}=\emptyset$. Así que, si $\alpha>\alpha_{n}$ para todo $n \in \omega$, entonces $W_{\alpha} \cap\left(\bigcup_{n<\omega} A_{n}^{*}\right)=\emptyset$. Elijamos un conjunto abierto-cerrado $O \subset W_{\alpha} \cap\left(\bigcup_{\alpha<\omega_{1}} U_{\alpha}\right)$. Como $O=\bar{B}-B=B^{*}$ para algún $B \subset A$, obtenemos que $B \cup B^{*}$ es compacto. Existe un compacto $K_{n_{0}}$ para el cual $B \cup B^{*} \subset K_{n_{0}}$ y por consiguiente $B \subset K_{n_{0}} \cap A=A_{n_{0}}$. Por lo tanto $B^{*} \subset A_{n_{0}}^{*}$, lo cual es contradictorio con la elección de $O$.

Para probar que $X$ es numerablemente compacto, supongamos que $D \subset X$ es un subespacio cerrado, discreto y numerable de $X$. De aquí resulta que $\bar{D}-D \subset$ $\omega^{*}-X=F \cup A$.

Consideremos la posibilidad $(\bar{D}-D) \cap A \neq \emptyset$. Si $a \in \bar{D}-D \cap A$, entonces tomemos un subconjunto abierto-cerrado $U \subset \omega^{*}$ tal que $\{a\}=U \cap A$, de donde $\{a\}=U \cap \bar{A}$. Puesto que el subconjunto cerrado $\bar{D} \cap U \subset \omega^{*}$ es no vacío, pues contiene al punto $a$, tenemos que es infinito. Por el teorema 1.42, obtenemos que $\bar{D} \cap U$ tiene cardinalidad $2^{\mathfrak{c}}$, lo cual implica que $(\bar{D} \cap U)-(D \cup\{a\}) \neq \emptyset$. Si $x \in(\bar{D} \cap U)-(D \cup\{a\})$ entonces $x \in(\bar{D}-D)-(F \cup A)$ lo que es una contradicción. 
De lo anterior se desprende que $(\bar{D}-D) \cap A=\emptyset$ y por lo tanto $\bar{D}-D \subset F$. Supongamos que $D^{\prime}=D-\bar{A}$ es infinito. Luego, $D^{\prime} \cap \bar{A}=\emptyset$ y también es válido que $A \cap \overline{D^{\prime}}=\emptyset$, pues $\overline{D^{\prime}}-D^{\prime} \subset \bar{D}-D \subset F \subset \omega^{*}-A$. Aplicando el teorema 1.47 tenemos que $\overline{D^{\prime}} \cap \bar{A}=\emptyset$, lo cual contradice que el conjunto no vacío $\overline{D^{\prime}}-D^{\prime}$ sea un subconjunto de $F \subset \bar{A}$. Por consiguiente, el conjunto $D-\bar{A}$ es finito. Por lo tanto el conjunto $E=D \cap \bar{A}=D \cap(\bar{A}-A) \subset X \cap(\bar{A}-A)=\bigcup_{\alpha<\omega_{1}} V_{\alpha}$ es infinito, ya que $E=D-(D-\bar{A})$. Como la familia $\left\{V_{\alpha}: \alpha<\omega_{1}\right\}$ es creciente y $E$ es numerable, tenemos que existe un $\alpha<\omega_{1}$ tal que $E \subset V_{\alpha}$. Por lo tanto $\bar{E}-E \subset \bar{E} \subset V_{\alpha}$, lo cual es contradictorio con $\bar{E}-E \subset \bar{D}-D \subset F$, ya que $F \cap V_{\alpha}=\emptyset$. Esta última contradicción prueba que el espacio $X$ es numerablemente compacto. 


\section{Conclusiones}

En el transcurso del presente trabajo, hemos obtenido algunos resultados nuevos en lo referente al tema de la completitud en espacios topológicos; además de algunas de sus aplicaciones en Algebra Topológica.

En el ejemplo 2.26 constatamos la no aditividad, en algunos modelos de ZFC, de la Čech completitud en potencias finitas. Nosotros probamos bajo $\mathrm{CH}$ (ejemplo 3.14) que existen espacios Čech completos y numerablemente compactos al mismo tiempo que no son ultracompletos, dando así respuesta a una pregunta planteada en el artículo [BY2].

Como consecuencia de nuestro estudio de estas propiedades, obtuvimos para el caso de los grupos topológicos, que la Čech completitud es invariante respecto a funciones abiertas (lema 2.32), se preserva bajo uniones finitas (teorema 2.29) y es equivalente a la casi Čech completitud (corolario 2.31). En lo que respecta a la ultracompletitud, probamos también para los grupos topológicos, que esta propiedad es equivalente a la compacidad local (teorema 3.10). 


\section{Bibliografía}

[A] R. Arens, A topology for spaces of transformations, Ann. of Math. 47 (1946), 480-495.

[AL] J.M. Aarts and D.J. Lutzer, Completeness properties designed for recognizing Baire spaces, Dissertationes Mathematicae CXVI, Warszawa (1974).

[AP] A.V. Arhangel'skiı̌, V.I. Ponomarev, Fundamentals of General Topology: Problems and Exercises, D. Reidel Publishing Company (1984).

[Ar1] A.V. Arhangel'skiu, On topological spaces which are complete in the sense of Čech, Cep. Mat., no. 2 (1961), 37-40.

[Ar2] A.V. Arhangel'skiur, Bicompact sets and the topology of spaces, Dokl. Akad. Nauk SSSR 150 (1963), 9-12.

[Ar3] A.V. Arhangel'skil, Bicompact sets and the topology of spaces, Trans. Mosc. Math. Soc. 13 (1965), 1-62.

[Ar4] A.V. Arhangel'skiu, Mappings and spaces, English translation: Russian Math. Surveys 21, no. 4 (1966), 115-162.

[Ar5] A.V. Arhangel'skil, Linear homeomorphisms of functions spaces, Dokl. Akad. Nauk SSS, vol 264, no. 6 (1982), 1289-1292.

[BGT] Z. Balogh, G. Gruenhage and V. Tkachuk, Additivity of metrizability and related properties, Topology and its Applications, vol. 84 (1998), 91-103.

[BY1] D. Buhagiar and I. Yoshioka, Ultracomplete topological spaces, en prensa

[BY2] D. Buhagiar and I. Yoshioka, Sums and products of ultracomplete topological spaces, Topology and its Applications, en prensa.

[Co] H. H. Corson, The determination of paracompactness by uniformities, Amer. J. Math. 80 (1958), 185-190.

[Cs] A. Császár, Strongly complete, supercomplete and ultracomplete spaces, Mathematical Structures-Computational Mathematical Modelling, Papers dedicated to Prof. L. Iliev's 60th Anniversary, Sofia, (1975).

[ČP] E. Čech, B. Pospíšil, Sur les espaces compacts, Publ. Fac. Sci. Univ. Masaryk Brno 258 (1938), 3-7. 
[CČN] J. Chaber, M. M. Čoban and K. Nagami, On monotonic generalizations of Moore spaces, Čech complete spaces and p-spaces, Fundamenta Mathematicae, vol. 84 (1974), 107-119.

[Cha] J. Chaber, Conditions which imply compactness in countably compact spaces, Bull. Acad. Pol. Sci., Ser. Sci. Math., vol. 24 (1976), 993-998.

[Ch] M. M. Choban, On completion of topological groups (en ruso), Vestnik Mosk. Univ. Matematika, N1 (1970), 33-38.

[En] R. Engelking, General Topology, Heldermann Verlag Berlin (1989).

[F] M. Fréchet, Sur quelques points du calcul fonctionnel, Rend. del Circ. Mat. di Palermo 22 (1906), 1-74.

[Fr1] Z. Frolík, An example concerning countably compact spaces, Czech. Math. Journ. 10 (1960), 255-257.

[Fr2] Z. Frolík, On the topological product of paracompact spaces, Bulletin de l'Académie Polonaise des Sciences, Série des Sciences Math., Astro. et Phys. VIII (1960), no. 11-12, 747-750.

[Fr3] Z. Frolík, Generalization of the $G_{\delta}$-property of complete metric spaces, Czech. Math. Journ. 10 (1960), 359-379.

[Fr4] Z. Frolík, Applications of complete families of continuos functions to the theory of Q-spaces, Czech. Math. Journ. 11 (1961), 115-133.

[Fr5] Z. Frolík, Locally topologically complete spaces, English translation: Soviet Math. Dokl. 2 (1961), 323-325.

[Fr6] Z. Frolík, Locally $G_{\delta}$-spaces, Czech. Math. Journ. 12 (1962), 346-355.

$[\mathrm{Fu}]$ Z. Furdzik, On absolute $G_{\delta}$ in perfectly normal topological spaces, Zeszyty Nauk. U. J. 167, Prace Mat. 12 (1968), 17-18.

[Ha] F. Hausdorff, Mengenlehre, Berlin (1927).

[Ho1] N. R. Howes, On completeness, Pacific J. Math. 38 (1971), 431-440.

[Ho2] N. R. Howes, Paracompactifications, preparacompactness and some problems of K. Morita and H. Tamano, Q \& A in General Topology 10 (1992), 191-204.

[Ho3] N. R. Howes, Modern Analysis and Topology, Springer-Verlag, (1995).

[Ju] I. Juhász, Cardinal functions in topology, Math. Centre Tracts 34 (1971), Amsterdam.

[Ka] S. Kakutani, Über die Metrisation der topologischen Gruppen, Proc. Imp. Acad. Tokyo 12 (1936), 82-84.

[Ke] J. L. Kelly, General Topology, New York (1955). 
[Ku] Kenneth Kunen, Set Theory. An Introduction to Independence Proofs, Studies in Logic and the Foundations of Mathematics, volume 102 (1980), North Holland Publishing Company.

[Lo] M. López de Luna, Some new results on Čech-complete spaces, Topology Proceedings, volume 24 (1999), Summer.

[Ma] S. Mazurkiewicz, Theory of $G_{\delta^{-}}$sets, Wektor 6 (1917-18), 129-185.

[Ma] S. Mazurkiewicz, Sur un ensemble $G_{\delta}$ punctiforme qui n'est homéomorphe à aucun ensemble linéaire, Fund. Math. 1 (1920), 61-81.

[Mi] E. Michael, Complete spaces and tri-quotient maps, Illinois J. Math. 21 (1977), 716-733.

[Na] J. Nagata, Modern General Topology, Amsterdam (1968).

[OY] H. Otha and K. Yamada, Simple examples showing that various topological properties are not finitely additive in the sense of V. V. Tkachuk, Math. Japonica, Vol. 47, N1, 93-95 (1998).

[Pa] B.A. Pasynkov, Almost metrizable topological groups (en ruso), DAN SSSR, vol 161, N2, 281-284 (1965).

[Po] L. Pontriagin, Topological Groups, Mir. (1977).

[PT] V.I. Ponomarev and V.V. Tkachuk, The countable character of $X$ in $\beta X$ compared with the countable character of the diagonal in $X \times X$, Vestnik Moskovskogo Universiteta. Matematika, Vol. 42, No. 5, 16-19 (1987).

[Ro] S. Romaguera, On cofinally complete metric spaces, Q \& A in General Topology 16 (1998), 165-169.

[Šo] A. P. Šostak, A characterization of the class of Čech-complete spaces as an $\mathcal{E}$-compactness class, Bull. Acad. Pol. Sci. Sér. Math. 22 (1974), 839-844.

[TkV] V.V. Tkachuk, Finite and countable additivity of topological properties in nice spaces, Trans. Amer. Math. Soc., vol. 341, N2 (1994), 585-601.

[TkM] M.G. Tkachenko, On a property of bicompacta, Seminar on General Topology, ed. by P.S. Alexandroff, Mosc. Univ. P.H., Moscow (1981), 149-156.

[TVHR] M.G. Tkachenko, L.M. Villegas Silva, C. Hernández García, O.J. Rendón Gómez, Grupos Topológicos, UAM-Iztapalapa, México, (1997).

[Va] H. Vaughan, On locally compact metrisable spaces, Bull. Amer. Math. Soc. 43 (1937), 532-535.

[Wa] R. C. Walker, The Stone-Čech compactification, Berlin (1974). 
[Wi] H.H. Wicke, On the Hausdorff open continuous images of Hausdorff paracompact p-spaces, Proc. Amer. Math. Soc. 22 (1969), 136-140.

[Ze] P. Zenor, On the completeness of the space of compact subsets, Proc. Amer. Math. Soc. 26 (1970), 190-192. 


\section{Lista de símbolos}

A continuación presentamos algunos símbolos utilizados en este trabajo. Se proporciona una breve descripción de cada símbolo y el número de la página en el cual se introduce.

$\bar{A}^{X}$, cerradura de $A$ en $X, \mathbf{8}$

$\beta \omega$, el espacio $\beta \omega, \mathbf{2 5}$

$\operatorname{cl}_{X}(A)$, cerradura de $A$ en $X, \mathbf{2 3}$

$\beta X$, la compactificación de Stone-Čech de un espacio de Tychonoff $X, \mathbf{7}$

CH, la hipótesis del continuo, 60

$\diamond^{+}$, axioma diamante más, 49

$\exp (X)$, familia de los subconjuntos

de $X, 6$

$f\lceil A$, restricción de la función $f$ al conjunto $A, \mathbf{1 5}$

$f(\mathcal{H})$, la familia $\{f(H)\}_{H \in \mathcal{H}}, \mathbf{2 0}$

$I$, el intervalo $[0,1]$ con

la topología natural, 7

$\bigcap \overline{\mathcal{F}}$, el conjunto $\bigcap_{F \in \mathcal{F}} \bar{F}, \mathbf{1 1}$

$\mathcal{T}^{*}(X)$, familia de subconjuntos abiertos y no vacíos de $X, \mathbf{2 0}$

$\mathcal{T}_{c}^{*}(X)$, familia de subconjuntos

abierto-cerrados y no vacíos de $X, \mathbf{6 0}$

$\mathcal{T}\left(x_{0}, X\right)$, familia de subconjuntos abiertos

de $X$ que contienen al punto $x_{0}, \mathbf{6}$

$\mathcal{U} \prec \mathcal{V}, \mathcal{U}$ es un refinamiento de $\mathcal{V}, 33$

$\bigcup \mathcal{U}$, la unión $\bigcup_{U \in \mathcal{U}} U, \mathbf{1 8}$

$\omega^{*}$, el residuo $\beta \omega-\omega, \mathbf{2 5}$
$X^{\omega}$, producto numerable de $X, \mathbf{4 6}$

$\chi(K, X)$, carácter de $K$ en $X, \mathbf{1 6}$

$x^{n}$, el punto $\left(x_{1}, x_{2}, \ldots, x_{n}\right), x_{i}=x, \mathbf{4 7}$ 